\title{
RHEOLOGICAL INVESTIGATIONS OF LATEX-SURFACTANT-ASSOCIATIVE THICKENER AQUEOUS SYSTEMS
}

\author{
A Thesis \\ presented to \\ the Faculty of California Polytechnic State University,
}

San Luis Obispo

\author{
In Partial Fulfillment \\ of the Requirements for the Degree \\ Master of Science in Polymers and Coatings \\ by
}

Bishop Isaiah Hammack

June 2019 
(C) 2019

Bishop Isaiah Hammack

ALL RIGHTS RESERVED 


\section{COMMITTEE MEMBERSHIP}

TITLE: Rheological Investigations of Latex-SurfactantAssociative Thickener Aqueous Systems

AUTHOR: $\quad$ Bishop Isaiah Hammack

DATE SUBMITTED: June 2019

COMMITEE CHAIR: $\quad$ Raymond Fernando, Ph.D.

Professor, Department of Chemistry and Biochemistry

COMMITEE MEMBER: John Hagen, Ph.D.

Professor, Department of Chemistry and

Biochemistry

COMMITTE MEMBER: $\quad$ Erik Sapper, Ph.D.

Assistant Professor, Department of Chemistry and

Biochemistry 


\begin{abstract}
Rheological Investigations of Latex-Surfactant-Associative Thickener Aqueous Systems Bishop Isaiah Hammack
\end{abstract}

Surfactants and Thickeners are both additives used in fully-formulated waterborne coatings to provide colloidal stability, thickening, and other functionality. The behavior of each ingredient in a coating must be understood and controlled to maintain colloidal stability as well as balance other desired properties of the liquid coating and the dry paint film. In this work, quaternary systems of Water-Latex-Thickener-Surfactant were investigated to further the understanding of their behavior in coatings. The thickener used was a well characterized, hydrophobically-modified, ethoxylated urethane (HEUR) with two $\mathrm{C}_{18}$ terminal hydrophobes and 795 average repeat units of ethylene oxide as the hydrophilic spacer. Two latexes, a hydrophobic butyl acrylate/styrene and a hydrophilic butyl acrylate/methyl methacrylate, each containing a small amount of methacrylic acid monomer were used. Six different surfactants, three non-ionic and three-anionic, were used. By maintaining the concentrations of latex and HEUR thickener as constants and varying the surfactant concentration, effects of the surfactant loading on rheology were determined. As concentration of surfactant increases, a characteristic shear-thickening maximum associated with bridging of latex particles by the HEUR thickener was seen to shift to higher shear rates; surfactants at all concentrations studied, except SDS, lowered the viscosities within the low shear rate region. Dynamic viscoelastic measurements shed further light into the behavior of the mixtures. The results will be explained based on surfactant and latex surface polarities and the competitive adsorption between the 
surfactant and HEUR hydrophobes, and other interactions between surfactants and thickeners. 


\section{ACKNOWLEDGMENTS}

Special Thanks for Financial Support Provided by:

Arthur C. Edwards Endowment

Golden Gate Society for Coatings Technology and the Margaret Hartmann Scholarship

Additional Thanks for Provided Resources and Materials:

Kenneth N. Edwards Western Coatings Technology Center

Members of the Dow Chemical Company Research Team:

Dr. Gary W. Dombrowski

Dr. John J. Rabasco

Dr. Patrick E. Hartnett 


\section{TABLE OF CONTENTS}

\section{Page}

LIST OF TABLES xi

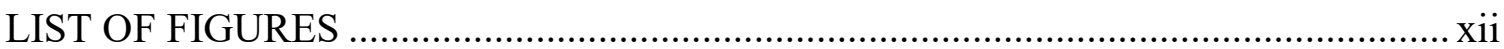

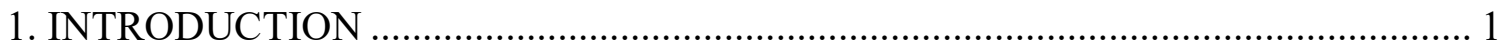

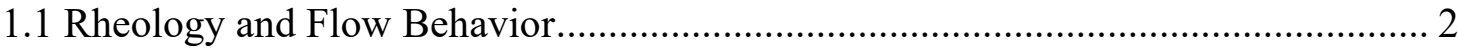

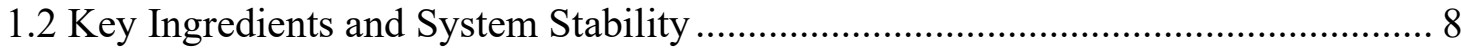

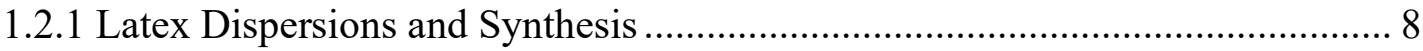

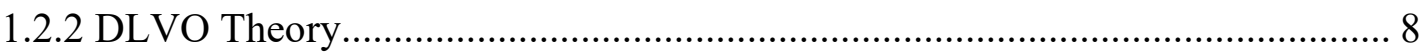

1.2.3 Steric and Volume Exclusion Repulsion................................................. 11

1.3 Thickeners and Potential Destabilization ............................................................... 12

1.3.1 Nonadsorbing Polymers and Depletion Flocculation ................................... 12

1.3.2 Associative Thickeners and Bridging Flocculation...................................... 14

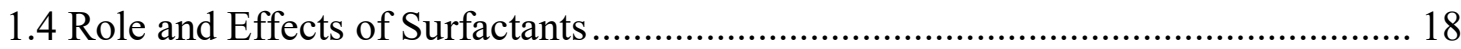

1.5 Relevant Research and Statement of Intent................................................ 19

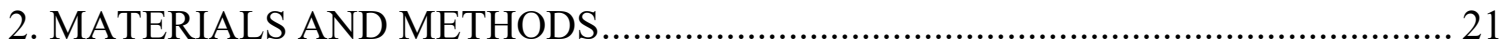

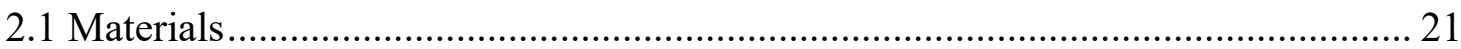

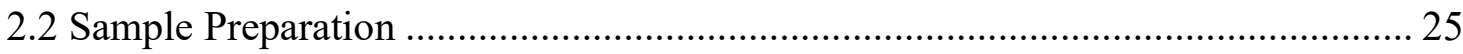

2.3 Rheological Testing Parameters ................................................................... 26

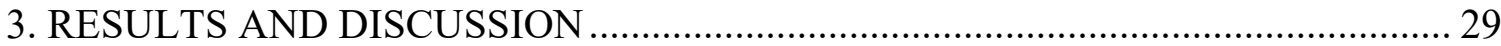


3.1 Flow Sweep Analysis of BA/STY-Surfactant-Thickener Aqueous Systems

3.1.1 BA/STY-HEUR Systems with $0.1 \mathrm{wt} \%$ Additional Surfactant 31

3.1.2 BA/STY-HEUR Systems with $0.25 \mathrm{wt} \%$ Additional Surfactant 34

3.1.3 BA/STY-HEUR Systems with $0.50 \mathrm{wt} \%$ Additional Surfactant 36

3.1.4 BA/STY-HEUR Systems with $0.75 \mathrm{wt} \%$ Additional Surfactant 39

3.1.5 BA/STY-HEUR Systems with $1.0 \mathrm{wt} \%$ Additional Surfactant 40

3.1.6 High shear viscosity analysis of BA/STY systems 41

3.2 Flow Sweep Analysis of BA/MMA-Surfactant-Thickener Aqueous Systems ....... 44

3.2.1 BA/MMA-HEUR Systems with $0.10 \mathrm{wt} \%$ Additional Surfactant 44

3.2.2 BA/MMA-HEUR Systems with $0.25 \mathrm{wt} \%$ Additional Surfactant 46

3.2.3 BA/MMA-HEUR Systems with $0.50 \mathrm{wt} \%$ Additional Surfactant 48

3.2.4 BA/MMA-HEUR Systems with $0.75 \mathrm{wt} \%$ Additional Surfactant 49

3.2.5 High shear viscosity analysis of BA/MMA systems 50

3.3 Comparative Flow Sweep Analysis of BA-STY and BA-MMA systems. 52

3.3.1 Comparison of BA-STY-HEUR and BA-MMA Systems with $0.1 \mathrm{wt} \%$

Additional Surfactant. 52

3.3.2 Comparison of BA-STY-HEUR and BA-MMA Systems with $0.25 \mathrm{wt} \%$ Additional Surfactant 54

3.3.4 Comparison of BA-STY-HEUR and BA-MMA Systems with $0.50 \mathrm{wt} \%$ Additional Surfactant. 55 
3.3.5 Comparison of BA-STY-HEUR and BA-MMA Systems with 0.75 wt\%

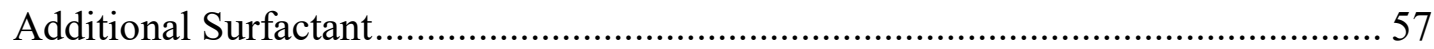

3.4 Dynamic Data Analysis of BA/STY-HEUR-Surfactant systems. ......................... 58

3.4.1 Dynamic Data for BA/STY-HEUR-Surfactant System with 0.10 wt\%

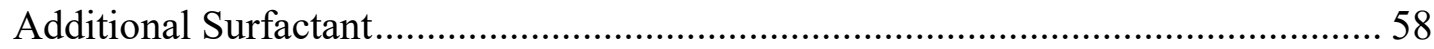

3.4.2 Dynamic Data for BA/STY-HEUR-Surfactant System with 0.25 wt\%

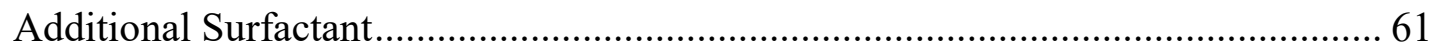

3.4.3 Dynamic Data for BA/STY-HEUR-Surfactant System with 0.50 wt\%

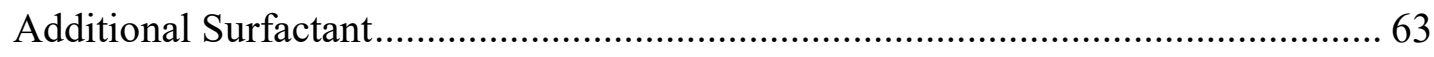

3.4.4 Dynamic Data for BA/STY-HEUR-Surfactant System with 0.75 wt\%

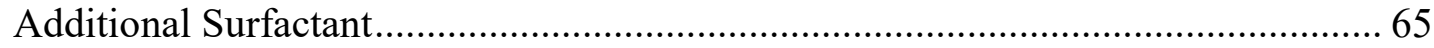

3.4.5 Dynamic Data for BA/STY-HEUR-Surfactant System with $1.0 \mathrm{wt} \%$ Additional

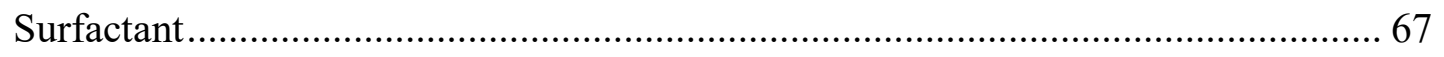

3.5 Dynamic Data Analysis of BA/MMA-HEUR-Surfactant systems. ................... 69

3.5.1 Dynamic Data for BA/MMA-HEUR-Surfactant System with $0.10 \mathrm{wt} \%$

Additional Surfactant ................................................................................ 70

3.5.2 Dynamic Data for BA/MMA-HEUR-Surfactant System with 0.25 wt\%

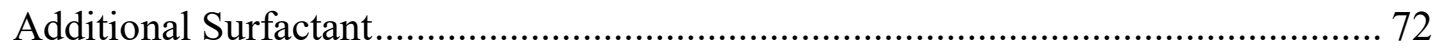

3.5.3 Dynamic Data for BA/MMA-HEUR-Surfactant System with 0.50 wt \%

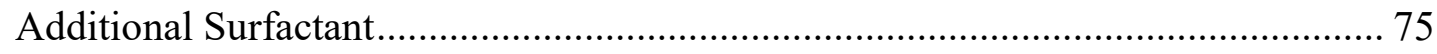


3.5.4 Dynamic Data for BA/MMA-HEUR-Surfactant System with $0.75 \mathrm{wt} \%$

Additional Surfactant.

77

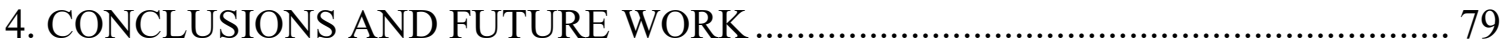

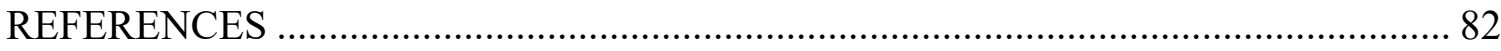




\section{LIST OF TABLES}

Table

1. Experimental Latex Properties (where BA is butyl acrylate, MMA is methyl

methacrylate, MAA is methacrylic acid). 23

2. Approximate molecular weight and critical micelle concentration values of the 6 surfactants. 25

3. Parameters for Flow Peak Hold of Waterborne Latex/HEUR/Surfactant Mixtures..... 26

4. Parameters for Flow Sweep of Waterborne Latex/HEUR/Surfactant Mixtures........... 27

5. Parameters for Logarithmic Amplitude Sweep of Waterborne Latex/HEUR/Surfactant

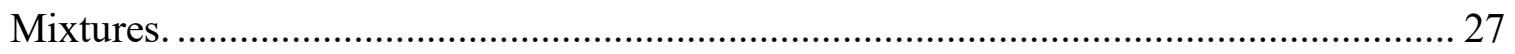

6. Parameters for Linear Amplitude Sweep of Waterborne Latex/HEUR/Surfactant

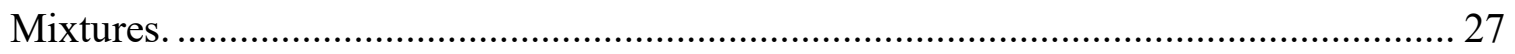

7. Parameters for Frequency Sweep of Waterborne Latex/HEUR/Surfactant Mixtures. . 28 


\section{LIST OF FIGURES}

Figure

Page

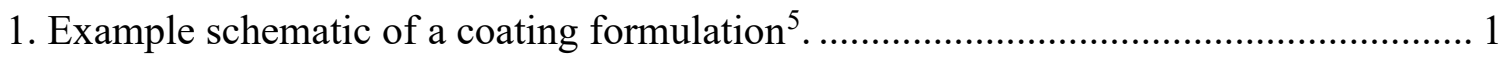

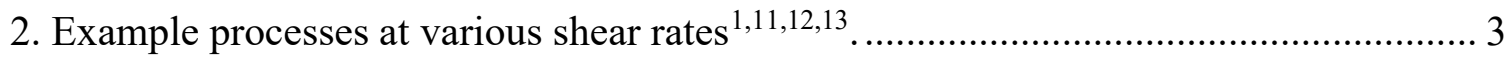

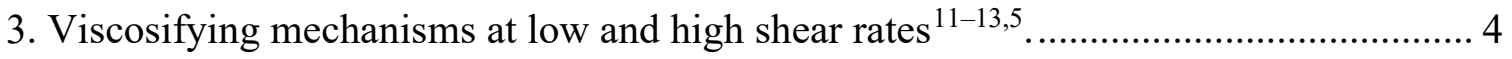

4. Examples of the three different viscosity profiles plotted as a function of shear rate $^{5} \ldots 5$

5. Representation of the Maxwell Model (a) at rest (b) showing the immediate elastic

response under stress, and (c) the slow viscous response under constant stress ${ }^{11,10 \ldots \ldots \ldots .6}$

6. Representation of three different stress responses where $0^{\circ}$ is in phase with the

induced oscillatory stress and $90^{\circ}$ is completely out of phase................................... 7

7. Examples of a stable suspension and an unstable suspension at different points in

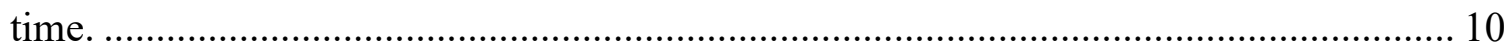

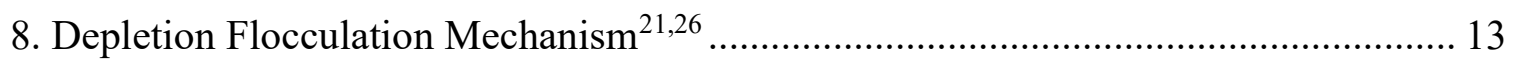

9. Bridging Flocculation by Direct Bridging Mechanism ....................................... 16

10. Bridging Flocculation by Indirect Bridging Mechanism ...................................... 17

11. Schematic of a generic surfactant molecule demonstrating the hydrophobic tail and

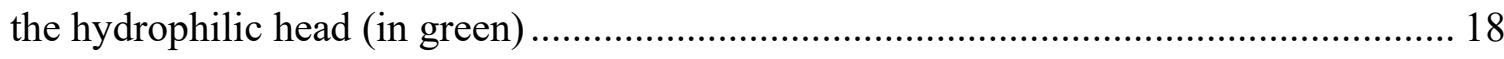

12. The 4 major types of surfactant as categorized by head type: a) nonionic, b)

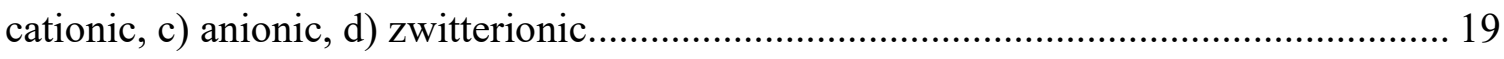

13. Dispersion phase diagrams of two BA/MMA-HEUR-SDS dispersions.................... 20

14. Structure of C18-EO795 HEUR Thickener. [n $=795, \mathrm{R}=\mathrm{C} 18, \mathrm{Mn}$ of $26.8 \mathrm{~kg}$

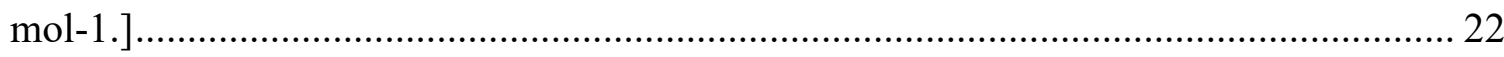


15. a) Tergitol 15-S-40 [n1+n2=11-15 and $n 3=41]$; b) Triton $X-100$ [n 9.5]; c) TSP-16 [n=16]; d) TSP-16S [n=16]; e) TSP-16PE30 [n=16, pH:0.9]; f) Sodium dodecyl sulfate.

16. Generic flow sweep to demonstrate the four major regimes.

17. Flow Sweep Overlay of BA/STY Latex at 25 vol. \%, C18-EO795 thickener at 0.5 wt $\%$, Surfactant at $0.1 \mathrm{wt} . \%$ systems.

18. Regimes I and II with linear $\mathrm{y}$-axis for C18-EO795/BA-STY, with $0.1 \mathrm{wt} \%$ surfactant systems.

19. Flow Sweep Overlay of BA/STY Latex at 25 vol. \%, C18-EO795 thickener at 0.5 wt $\%$, Surfactant at 0.25 wt. $\%$ systems.

20. Regimes I and II with linear y-axis for C18-EO795/BA-STY, with $0.25 \mathrm{wt} \%$ surfactant systems.

21. Flow Sweep Overlay of BA/STY Latex at 25 vol. \%, C18-EO795 thickener at 0.5 wt $\%$, Surfactant at 0.50 wt. $\%$ systems. 36

22. Zoomed-in Regimes I and II with for C18-EO795/BA-STY, with $0.25 \mathrm{wt} \%$ surfactant systems. 38

23. Flow Sweep Overlay of BA/STY Latex at 25 vol. \%, C18-EO795 thickener at 0.5 wt. \%, Surfactant at 0.75 wt. \% systems.

24. Flow Sweep Overlay of BA/STY Latex at 25 vol. \%, C18-EO795 thickener at 0.5 wt. $\%$, Surfactant at $1.0 \mathrm{wt} . \%$ systems. 40 25. Viscosity values at $100 \mathrm{~s}-1$ as a function of surfactant concentration (wt. \%) for BA/STY systems 
26. Viscosity values at $1000 \mathrm{~s}-1$ as a function of surfactant concentration (wt. \%) for BA/STY systems.

27. Flow Sweep Overlay of BA/MMA Latex at 25 vol. \%, C18-EO795 thickener at 0.5 wt. $\%$, Surfactant at 0.1 wt. $\%$ systems. 44

28. Regimes I and II with linear y-axis for C18-EO795/BA-MMA, with $0.10 \mathrm{wt} \%$ surfactant systems. 45

29. Flow Sweep Overlay of BA-MMA Latex at 25 vol. \%, C18-EO795 thickener at 0.5 wt. \%, Surfactant at 0.25 wt. \% systems. 46 30. Flow Sweep Overlay of BA-MMA Latex at 25 vol. \%, C18-EO795 thickener at 0.5 wt. $\%$, Surfactant at 0.50 wt. $\%$ systems. . 48

31. Flow Sweep Overlay of BA-MMA Latex at 25 vol. \%, C18-EO795 thickener at 0.5 wt. \%, Surfactant at 0.75 wt. \% systems.

32. Viscosity values at $100 \mathrm{~s}-1$ as a function of surfactant concentration (wt. \%) for BA/MMA systems 50

33. Viscosity values at $1000 \mathrm{~s}-1$ as a function of surfactant concentration (wt. \%) for BA/MMA systems 51

34. Flow sweep comparison of BA-STY and BA-MMA latex at 25 vol. \%, C18-EO795 thickener at $0.5 \mathrm{wt} \%$, surfactant at $0.1 \mathrm{wt} . \%$ systems. 52

35. Flow sweep comparison of BA-STY and BA-MMA latex at 25 vol. \%, C18-EO795 thickener at $0.5 \mathrm{wt} \%$, surfactant at $0.25 \mathrm{wt} . \%$ systems. 54 36. Flow sweep comparison of BA-STY and BA-MMA latex at 25 vol. \%, C18-EO795 thickener at $0.5 \mathrm{wt} \%$, surfactant at $0.50 \mathrm{wt} . \%$ systems. 
37. Flow sweep comparison of BA-STY and BA-MMA latex at 25 vol. \%, C18-EO795 thickener at $0.5 \mathrm{wt} \%$, surfactant at $0.75 \mathrm{wt} . \%$ systems. 57

38. Linear amplitude sweep storage moduli overlay of BASTY-HEUR-Surfactant system at $0.1 \mathrm{wt} \%$ of additional surfactant. 59 39. Phase angle plot for BASTY-HEUR-Surfactant system at $0.1 \mathrm{wt} \%$ of additional surfactant. 60

40. Linear amplitude sweep storage moduli overlay of BASTY-HEUR-Surfactant system at $0.25 \mathrm{wt} \%$ of additional surfactant. 61

41. Phase angle plot for BASTY-HEUR-Surfactant system at $0.25 \mathrm{wt} \%$ of additional surfactant

42. Linear amplitude sweep storage moduli overlay of BASTY-HEUR-Surfactant system at $0.50 \mathrm{wt} \%$ of additional surfactant. 64

43. Phase angle plot for BASTY-HEUR-Surfactant system at $0.50 \mathrm{wt} \%$ of additional surfactant. 65

44. Linear amplitude sweep storage moduli overlay of BASTY-HEUR-Surfactant system at $0.75 \mathrm{wt} \%$ of additional surfactant. 66

45. Phase angle plot for BASTY-HEUR-Surfactant system at $0.75 \mathrm{wt} \%$ of additional surfactant. 67

46. Linear amplitude sweep storage moduli overlay of BASTY-HEUR-Surfactant system at $1.0 \mathrm{wt} \%$ of additional surfactant. 68

47. Phase angle plot for BASTY-HEUR-Surfactant system at $1.0 \mathrm{wt} \%$ of additional surfactant. 69 
48. Linear amplitude sweep storage moduli overlay of BA/MMA-HEUR-Surfactant system at 0.10 wt. $\%$ of additional surfactant. ......................................................... 71 49. Phase angle plot for BA/MMA-HEUR-Surfactant system at $0.10 \mathrm{wt}$. \% of additional surfactant 72

50. Linear amplitude sweep storage moduli overlay of BA/MMA-HEUR-Surfactant system at 0.25 wt. $\%$ of additional surfactant. 73 51. Phase angle plot for BA/MMA-HEUR-Surfactant system at $0.25 \mathrm{wt}$. $\%$ of additional surfactant. 74

52. Linear amplitude sweep storage moduli overlay of BA/MMA-HEUR-Surfactant system at $0.50 \mathrm{wt} . \%$ of additional surfactant.

53. Phase angle plot for BA/MMA-HEUR-Surfactant system at $0.50 \mathrm{wt}$. \% of additional surfactant 76

54. Linear amplitude sweep storage moduli overlay of BA/MMA-HEUR-Surfactant system at 0.75 wt. $\%$ of additional surfactant. 77 55. Phase angle plot for BA/MMA-HEUR-Surfactant system at $0.75 \mathrm{wt}$. \% of additional surfactant 78 


\section{INTRODUCTION}

Paints and coatings are essential technologies in almost every aspect of our lives. Coating technologies are a part of everything from artwork to maritime vessels, from fences to circuitry. When specifically looking into the subcategory of paints, their applications include homes, cars, signs, and many more. Diving even further, just latexbased waterborne paints alone have a wide range of applications from interior aesthetics to exterior environmental protection ${ }^{1,2}$. Paints and coatings are applied in so many aspects of the world because of their ability to provide necessary functions like corrosion resistance, energy efficiency, decoration, and other useful properties ${ }^{2,3}$. Paints are highly complex and have an infinite number of combinations of ingredients that can be included, but these can be simplified and broken down into five important categories. The five major ingredient categories are the resin (polymeric latex particles), the solvent or liquid medium, the pigment, the additives and the fillers ${ }^{1,2,3,4}$.

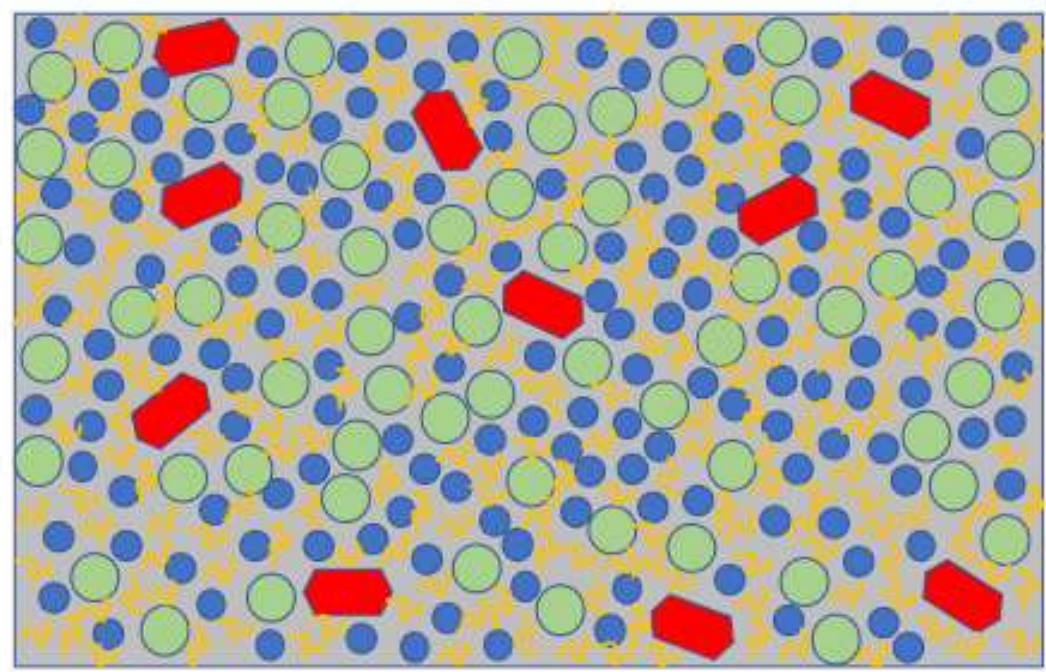

Latex Particle Pigment Particle

Filler Particle Polymer Chain

Figure 1. Example schematic of a coating formulation ${ }^{5}$. 
The liquid medium holds everything, ideally, in a stable dispersion. It also allows the pigments and resins to bind to each other in the drying and film formation process. The resin becomes the continuous coating that binds to the substrate. The pigment provides hiding power, barrier properties, and color to the paint ${ }^{4}$. The additives provide additional desired properties to the paint. This can range from the inhibition of mold growth, to scratch resistance, to specific viscosity profiles. Additives can include surfactants like emulsifiers, wetting agents, or dispersants ${ }^{2,3,6}$, or thickeners of various types $^{2,3,7}$. Finally, fillers are solid particles added to paint formulations such as calcium carbonate, talc, or silica, that take up additional volume, improve liquid coating and dry paint film properties, and often reduce the overall cost of production ${ }^{3,8}$. Each ingredient plays an important role and must be carefully measured to maintain a stable, functional, and affordable paint.

\subsection{Rheology and Flow Behavior}

Rheology is the field of study focused on the flow and deformation of materials ${ }^{1,9}$. Rheological analysis can be used to examine the continuum of materials from ideal viscous materials to ideal elastic materials and viscoelastic materials that fall in between these two extremes ${ }^{10}$. Most commercial products such as cosmetics, food products, and latex paints fall in this range between the two ends of the continuum ${ }^{10}$.

One means of studying the flow behavior of materials is through the monitoring and examination of the viscosity of the fluid. Viscosity, represented by $\eta$ (units of $\mathrm{Pa}^{*} \mathrm{~s}$ ), can be calculated from the ratio of shear stress $\sigma$ (units of Pa) divided by shear rate $\dot{\gamma}$ (units of $\mathrm{s}^{-1}$ ) (Equation 1) $)^{11,12}$. 


$$
\eta=\frac{\sigma}{\dot{\gamma}}
$$

The shear stress is the flow-inducing directional force applied to a fluid. The shear rate is the gradient of the velocity across the layers of the fluid ${ }^{1,2,10,12}$. Fully formulated latex paints are unique in the sense of the wide variety of processes to which they are subjected. Examples of associated shear rates for these processes can be seen in Figure 2.

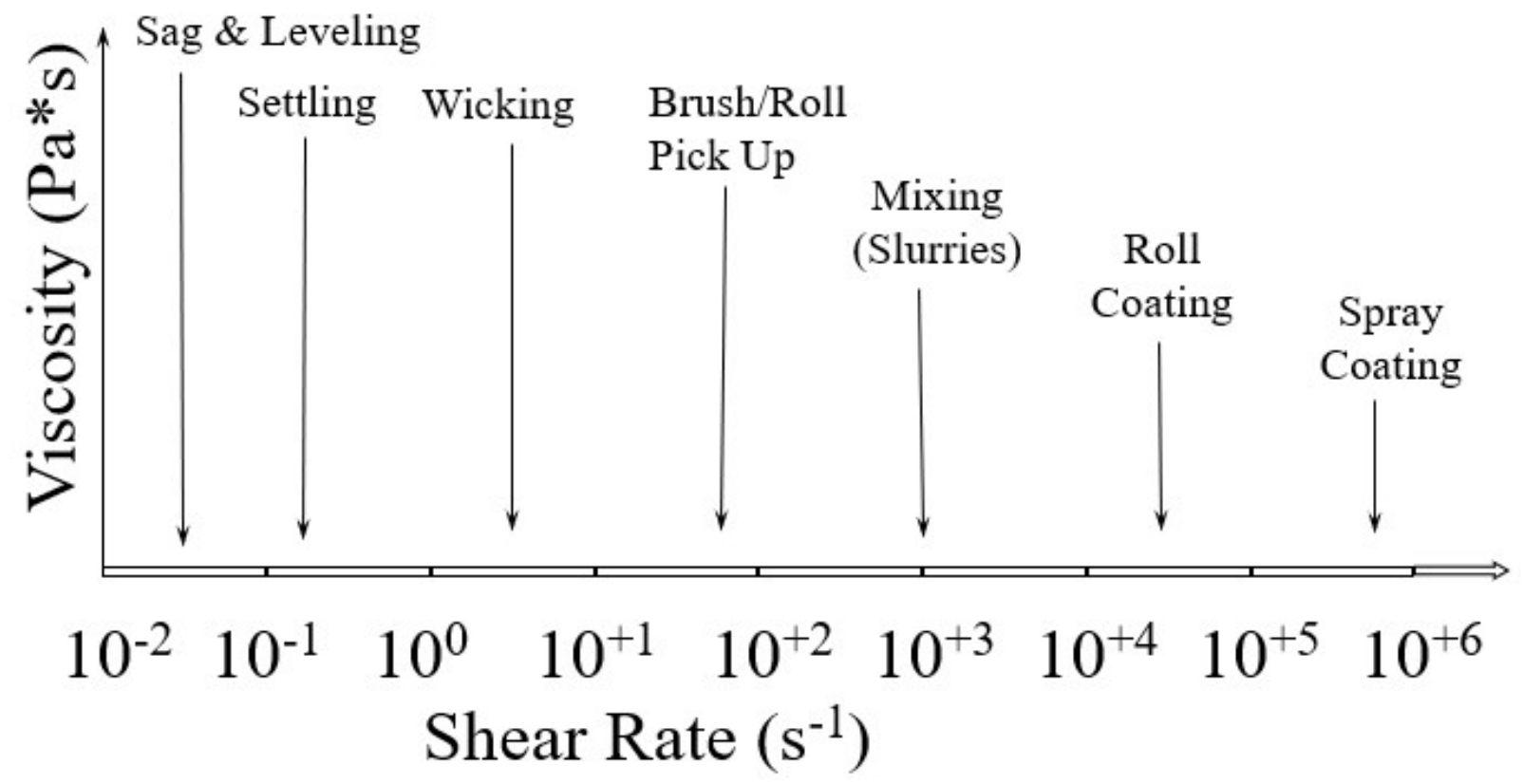

Figure 2. Example processes at various shear $\operatorname{rates}^{1,11,12,13}$.

Depending on the shear rate at which a fluid system is probed, different viscosifying mechanisms will be at work as can be seen in Figure 3. Due to the importance of both the variety of application methods and viscosifying mechanisms, viscosity is measured across a range of shear rates and plotted in what is referred to as a flow sweep or flow plot ${ }^{10}$. This type of measurement is acquired using a rotational test with known geometry cones and/or plates. When a rotational test is performed while 
controlling the shear rate, it can simulate flow velocity dependent processes like the ones mentioned in Figure 2 above ${ }^{10}$.

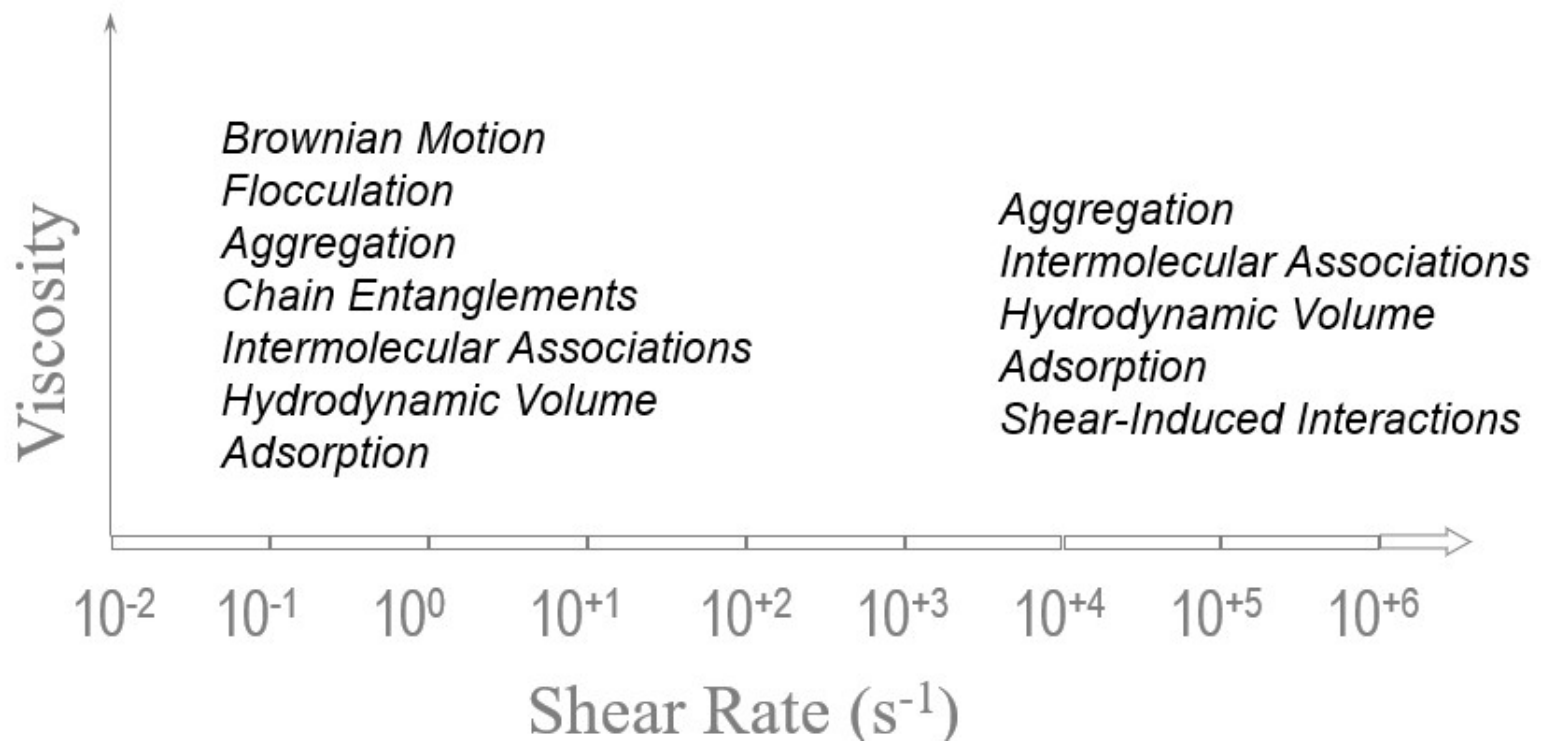

Figure 3. Viscosifying mechanisms at low and high shear rates ${ }^{11-13,5}$.

When measuring viscosity across a range of shear rates, there are several patterns that arise. Fluids can be categorized based on their viscosity behavior under different shear rates. Three general fluid behaviors can be seen in Figure 4. Newtonian fluids, like water or honey, maintain the same viscosity at any given shear rate. Fluids that show an increase in viscosity as shear rate increases are referred to as shear-thickening. Conversely, a fluid that shows a decrease in viscosity as shear rate increases is referred to as shear-thinning. However, not all fluids fall cleanly into one of these three categories. Often in more complex non-Newtonian systems, like a fully formulated paint, there can be combinations of shear thinning, shear thickening, and Newtonian regions ${ }^{12-14}$. 


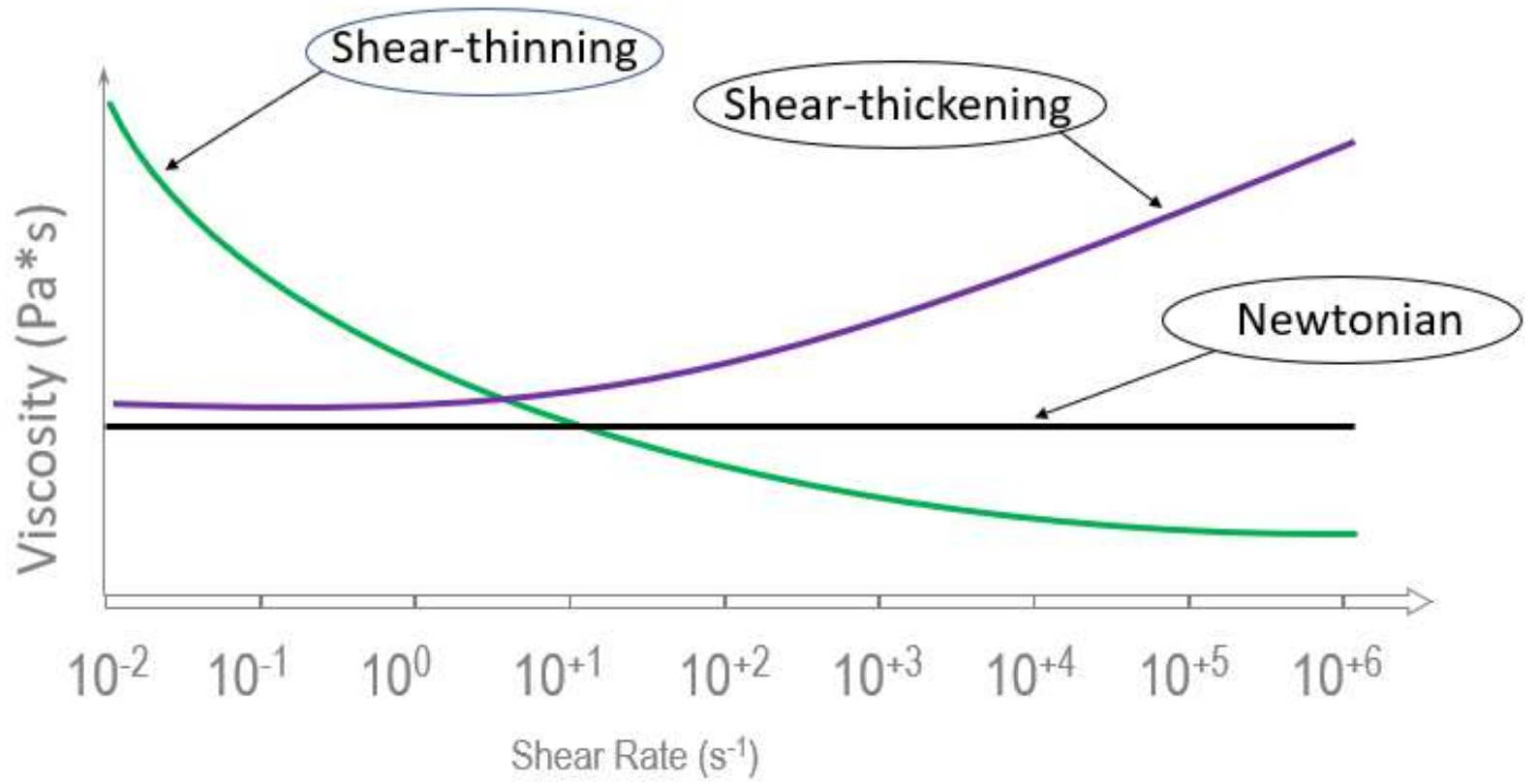

Figure 4. Examples of the three different viscosity profiles plotted as a function of shear rate $^{5}$.

Beyond direct measurements of viscosity at various shear rates, there is another way to define and quantify various materials from solids to liquids. Fully formulated paints and other systems with network-like structures do not behave like ideal elastic solids or viscous liquids, but rather as a combination of both attributes and are defined as being viscoelastic (cite TA instruments guide here). For the scope of the quaternary systems explored in this work, the Maxwell model can be applied. When HEURthickened latex systems are subjected to a strain, they can respond elastically immediately, but are also able to respond viscously and relax. In the Maxwell model, the viscoelastic response is represented by a spring and dashpot in series as represented in Figure 5. 


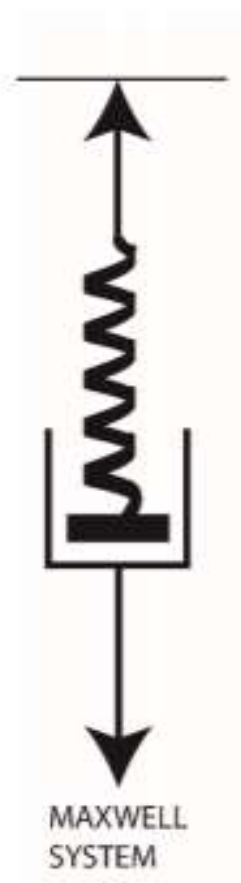

(a)

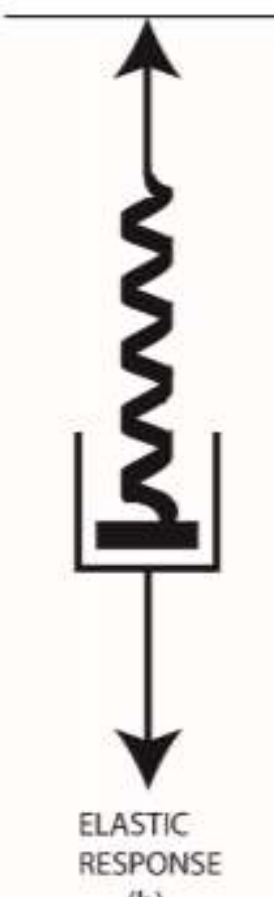

(b)

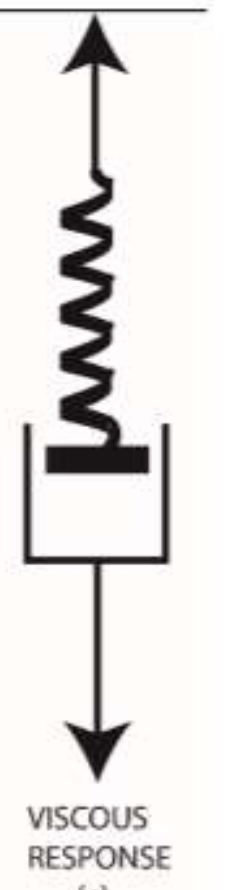

(c)

Figure 5. Representation of the Maxwell Model (a) at rest (b) showing the immediate elastic response under stress, and (c) the slow viscous response under constant stress ${ }^{11,10}$.

The elastic portion of the stress response, represented by the spring, is rapid and does not reverse until the stress is released. The viscous portion is represented by the slow response of the dashpot under continuous stress. When these are placed in series, the elastic behavior takes effect first and the spring stretches, then while the stress is held constant, the dashpot responds slowly, allowing the spring to return to its unstretched state. With this combination of responses, the total stress on the system eventually reaches zero ${ }^{1,10,11}$.

In order to measure and quantify the viscoelastic behavior of a fluid, a sinusoidal oscillatory force can be applied. This can be an oscillatory strain or oscillatory frequency, and each will reveal different information than a flow sweep, as these tests better 
simulate force-dependent processes, like required start up forces ${ }^{9,15,16}$. In a test where the strain is varied and the frequency is held constant, that is referred to as an amplitude or strain sweep. In a test where frequency is varied over a constant strain, it is called a frequency sweep. When the sinusoidal oscillatory force is applied, the system can respond in a variety of ways ${ }^{15,16}$.

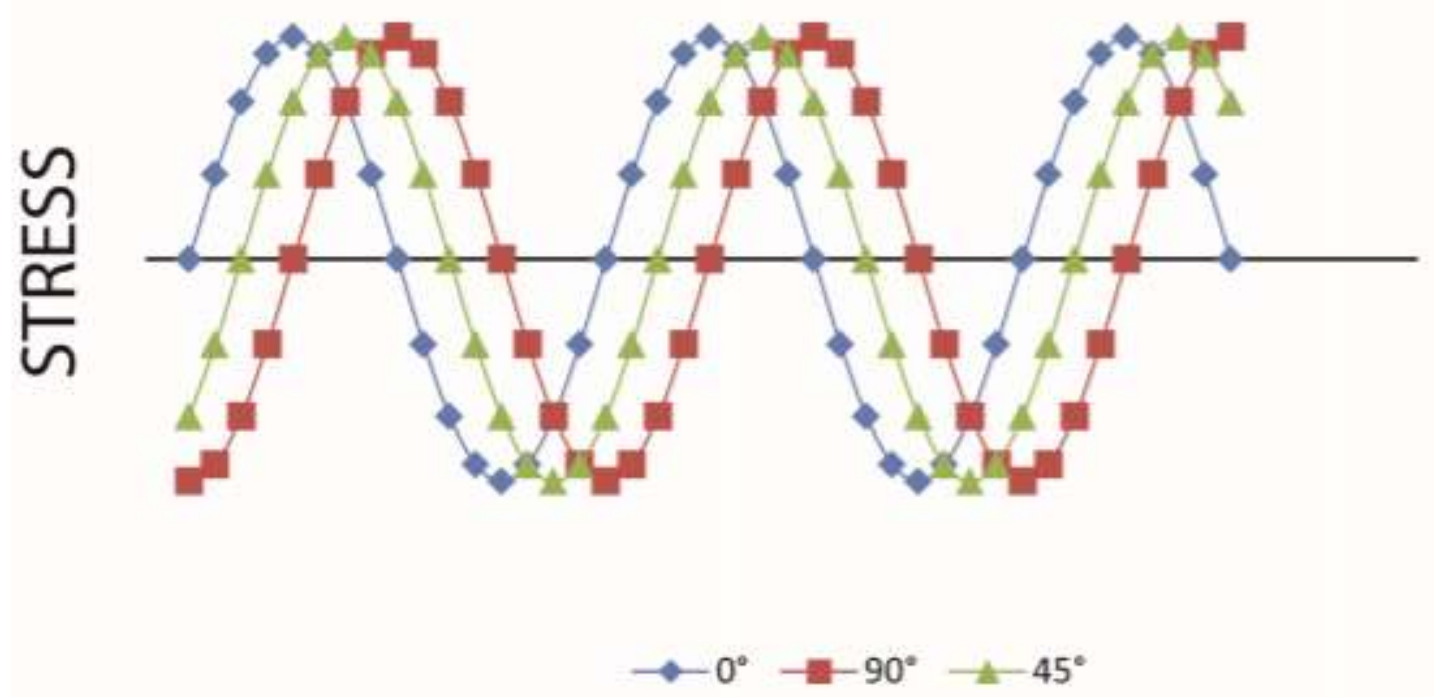

Figure 6. Representation of three different stress responses where $0^{\circ}$ is in phase with the induced oscillatory stress and $90^{\circ}$ is completely out of phase.

The time until a system responds can be represented by the phase angle, which shows how far out of phase the response is from the initial stress. If the system responds immediately like an ideal solid, then it is $0^{\circ}$ out of phase with the applied stress. At the other end of the continuum, if the material is $90^{\circ}$ out of phase when it responds to the stress, it is behaving like an ideal viscous liquid. Anywhere in between $0^{\circ}$ and $90^{\circ}$ is considered to be viscoelastic ${ }^{3,15,16}$. 


\subsection{Key Ingredients and System Stability}

\subsubsection{Latex Dispersions and Synthesis}

A colloidal dispersion is a system consisting of two major phases that are heterogeneously mixed. The two phases are referred to as the dispersion medium as the bulk phase and the dispersed phase which is made up of solid particles ${ }^{3}$. In a colloid, the Brownian motion is sufficient to overcome gravitational (or buoyancy depending on density) forces to remain stable. Colloidal dispersions include common things like milk, lotion, mayonnaise, or even latexes, but are not limited to just these examples. For the scope of this work, only latex dispersions will be discussed. A latex is made up of polymer particles (ranging in size from tens of nanometers to just a few microns in diameter) dispersed in water ${ }^{3}$.

Many latex dispersions are typically synthesized using free radical, chain-growth polymerization methods where monomeric species are emulsified in water. For this to occur, emulsion polymerizations require a few key ingredients to react including the bulk medium of water, the monomeric species of choice, some surfactant for micelle formation and particle stabilization, and initiator for radical formation ${ }^{2}$.

\subsubsection{DLVO Theory}

In a colloidal dispersion, there are a variety of mechanisms and forces at work. These include volume exclusion repulsion/steric forces, and electrostatic interactions and van der Waals forces. The primary theory that has been used for colloidal stability and adsorption discussions for many decades is known as DLVO theory, named after the four contributing scientists: Boris Derjaguin, Lev Landau, Evert Verwey, and Theodoor Overbeek $^{17,18}$. This theory is used to explain aggregation and stabilization of particles in 
liquid mediums and includes two major forces, one attractive force (van der Waals), and the other a repulsive force (electrostatic repulsion).

Van der Waals forces are a collection of intermolecular forces referring to the combination of dipole-dipole, dipole induced dipole (Debye), and London dispersion forces. The Keesom interaction is the attraction between two permanent dipoles ${ }^{17}$. The Debye force is the interaction known as induction where one permanent dipole induces a dipole on another surface or particle leading to an attractive interaction ${ }^{17}$. London dispersion forces are the attractive interactions between two molecules when instantaneous dipoles occur randomly ${ }^{17,6}$. Both the Debye and the London dispersion forces are attractive regardless of molecule or particle orientation while the other two forces are dependent on spatial arrangement. These forces together are additive and all short-range forces. In a fully formulated paint, these forces are only applicable on the local particle scale and do not create a significant effect on long range particles in a suspension.

Electrostatic interactions include both repulsion and attraction due to accumulation of charges across a particle. When particles are suspended in an aqueous environment, it is common for them to carry a surface charge. This charge can originate from ionic substitution with salts in the aqueous phase or from surface functional groups $^{17,19}$. At the charged surface of these particles, free-floating ions of a counter charge can associate into an electric double layer. In the case of a negatively charged particle, a layer of positive ions will surround the particle. As ions associate around the particle, they arrange into two layers. The closest layer to the particle surface is the Helmholtz layer and is most densely packed with strongly bonded ions. A second layer 
forms around this one known as the diffuse layer, consisting of loosely associated ions ${ }^{17,3}$. Together the Helmoltz layer and the diffuse layer are referred to as the electric double layer ${ }^{3,20}$. With sufficient ionic strength, this electric double layer can create an electrostatic repulsion away from other particles or regions of similar charge sign ${ }^{3,17,20}$.

DLVO theory states that there is a total potential energy of a system that can be described as a combination or sum of the attractive potential and the repulsive potential ${ }^{20}$. In a system with particles of identical or highly similar size, there are three major total potential regions. The first is at the closest length scale of just a nanometer or two called the primary minimum where attractive forces dominate. As the distance between particles grows to a few more nanometers it reaches a maximum where often repulsive forces dominate. After this peak it dips into a shallow minimum known as the secondary minimum ${ }^{3}$.

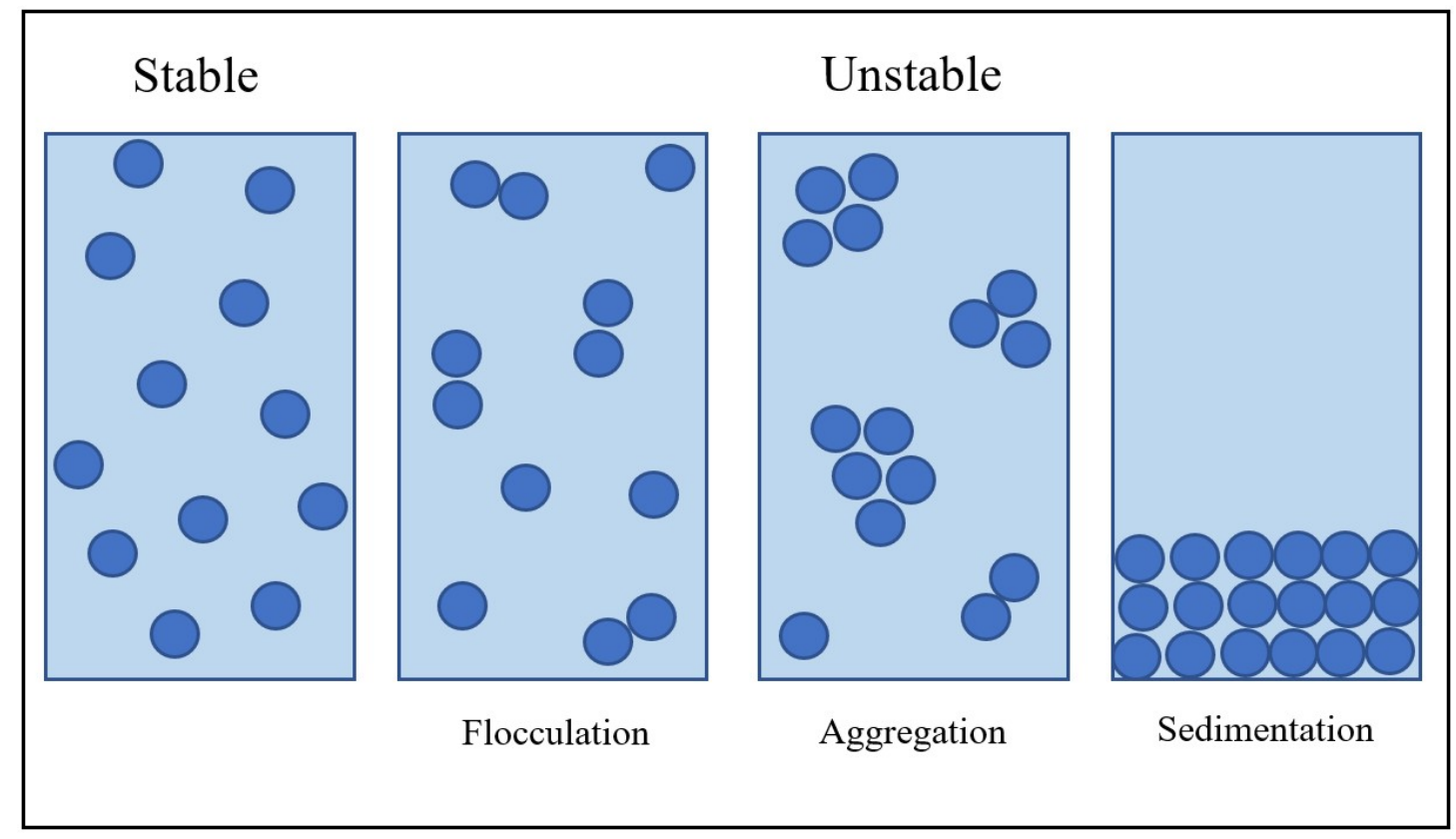

Figure 7. Examples of a stable suspension and an unstable suspension at different points in time. 
When repulsive forces outweigh the total effects of all the attractive forces between particles for an extended period of time, a stable dispersion is formed, however this stability is not guaranteed to be permanent in all cases. When the net forces in a suspension are highly repulsive, this is a kinetically stable dispersion ${ }^{3}$. However, if the net force is attractive in the slightest bit, then the dispersion will not be stable. Depending on the magnitude of the attractive force, instability can lead to complete separation of solids given enough time as demonstrated by the schematic in Figure 7. At early time scales, particles will attract to one another in pairs or triplets ${ }^{21}$. As time passes, these flocs will begin to aggregate, forming larger particles. Eventually flocs will grow to sufficient size to fall out of suspension, leading to the final stage of an unstable suspension known as sedimentation.

\subsubsection{Steric and Volume Exclusion Repulsion}

DLVO theory is thorough and accurately handles systems with symmetrical spherical particles in relatively dilute systems, but there are more forces at play in a more concentrated latex dispersion system ${ }^{3}$. Since there are more than electrolytes and spherical particles in a latex dispersion, it is important to understand some of the additional forces at play with polymeric species. This is where the combined effects of excluded volume and steric forces come into play.

Excluded volume repulsion is a concept in polymer science where one part of any long molecule or large particle cannot overlap with another portion of the same molecule. Additionally, in a multi-particle system this excluded volume is the volume that no other molecule can access due to the presence of the first molecule. These volume exclusion repulsive forces are relatively long-range forces and often outweigh short range attractive 
forces ${ }^{22}$. Repulsive steric forces are complex and are affected by several factors such as polymer density or type of solvent but are always entropically driven. Steric forces depend on the arrangement of molecules and the associated energy costs of multiple molecules existing in close proximity to one another. As a polymer chain is approached by another chain or surface, the number of available conformations or arrangements decreases, thus decreasing entropy of the given chain. Given that an isolated system will always strive for maximum entropy, the polymeric species will repel away from each other $^{17,22}$.

\subsection{Thickeners and Potential Destabilization}

\subsubsection{Nonadsorbing Polymers and Depletion Flocculation}

Thickeners or viscosity modifiers are generally categorized as nonadsorbing or associative, but there is an entire continuum of adsorption strength between nonadsorbing and permanently associating thickeners. Thickeners at different ends of this continuum function by different mechanisms which leads to different viscosity profiles and applications ${ }^{23}$. Nonadsorbing thickeners are rheology modifiers that rely on entanglements, flocculation, and volume exclusion from extremely high molecular weight polymers ${ }^{24}$. These thickeners sometimes are water soluble and swell, taking up space, creating an increase in viscosity, especially at low shear rates ${ }^{25}$. As shear rate increases with this type of thickener, viscosity decreases in a non-Newtonian, shearthinning behavior ${ }^{11}$. The degree to which the viscosity increases is related to both the concentration and the molecular weight of the cellulosic thickener (citation). However, at sufficient concentrations of added non-associating polymers, the system can become unstable. 
The destabilization that occurs with nonadsorbing polymers is known as depletion flocculation ${ }^{17}$. Non-associative polymers are often added into formulations in order to help stabilization or alter viscosity profiles, but at the right combinations of thickener concentration and molecular weight, depletion flocculation can happen ${ }^{3}$. This process begins when water-soluble polymers move from regions between particles, where movement and conformational freedom are restricted, to more open regions in the bulk of the solution. Depletion is an attractive force that occurs when the concentration of a polymer with no attraction to the latex in the bulk sufficiently exceeds the concentration of particles in the interparticle spaces ${ }^{21}$. (This model only accounts for systems in which steric stabilization is not considered, but the effect is still significant.)

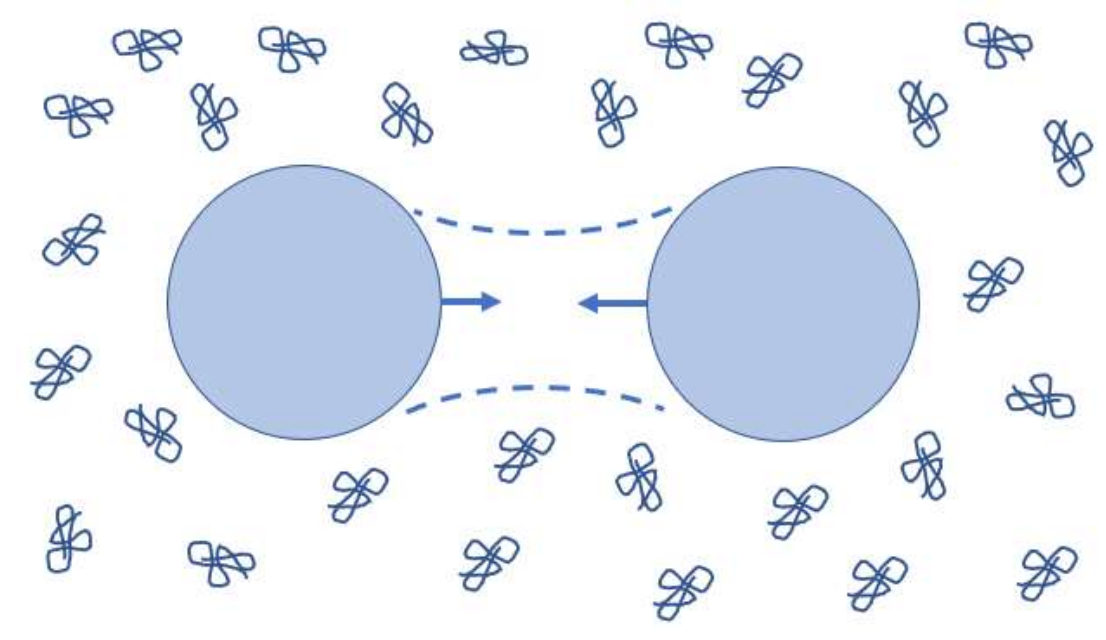

Figure 8. Depletion Flocculation Mechanism ${ }^{21,26}$

When the polymer in bulk reaches a high enough relative concentration, water molecules are driven out by osmotic pressure from the low polymer concentration regions to the high concentration regions in the bulk ${ }^{20}$. This creates an attractive force between 
latex particles and the osmotic pressure forces particles together into flocs and eventually into larger aggregates ${ }^{21}$.

\subsubsection{Associative Thickeners and Bridging Flocculation}

The other category of rheology modifiers are associative thickeners. These generally are water-soluble molecules, but the major difference from non-associative thickeners is the inclusion of non-polar or hydrophobic regions. These non-polar regions of the polymer are able to associate through hydrophobic interactions with other nonpolar or hydrophobic species in the dispersion. These other species may include other non-polar thickener ends, surfactant micelles, or latex particles ${ }^{27}$. Increases in viscosity arise from the formation of a complex transient network of associative bridges formed between the variety of species in the dispersion ${ }^{28}$. As the network of associations is formed, the motion of latex particles is reduced. This method of thickening makes the dispersion non-Newtonian, as viscosity becomes dependent on shear rate, as sufficiently high shear rates can disrupt associative behavior ${ }^{5,29}$. With associative thickeners it is not uncommon to see varied regions of Newtonian, shear-thickening, and shear-thinning behaviors $^{25}$.

One category of associative thickeners that are highly desirable in paint formulations are hydrophobically-modified ethoxylated urethanes (HEUR's). HEUR thickeners can have a wide variety of structures, but for the scope of this work only telechelic HEUR molecules will be examined. Telechelic HEUR's are poly(ethylene oxide) (PEO) chains that have been modified with non-polar associative ends through a urethane linkage ${ }^{11,13}$. Generally, HEUR's are low enough in molecular weight to disregard potential chain entanglement effects and are water soluble at a wide range of 
pH's making them ideal for waterborne systems ${ }^{30}$. There are several key traits and behaviors of HEUR thickeners in aqueous systems that are relevant to the scope of this work.

It has been shown that both the length of the hydrophobes and the overall molecular weight are relevant to the associative strength of the polymer ${ }^{31}$. As molecular weight increases, the length of the terminal hydrophobes relative to the remaining polymer grows smaller, so when compared to a lower molecular weight HEUR, there will be a lower number of active bridges ${ }^{32}$. Telechelic HEUR thickeners have also been observed at low concentrations in water to form a loop structure as the two terminal hydrophobes associate with one another ${ }^{11}$. As concentration of thickener is increased beyond the critical micelle concentration, then the HEUR molecules begin to form flower-like micelles ${ }^{11,33}$. In these flower micelles, most associative ends are found in the hydrophobic center, but some hydrophobes can be found dangling out into the bulk ${ }^{11,33}$. Further increases of concentration above the critical aggregation concentration lead to the formation of a 3-dimensional network between these flower-like micelles ${ }^{11,34,35}$. Because of these behaviors, HEUR thickeners have the potential for highly tailorable viscosity profiles through alterations in molecular weight, hydrophobe length, and concentration, but without careful consideration for associative strength new issues of destabilization can arise.

When working with associative polymers with high enough associative affinity for the surface of latex particles, another form of destabilization known as bridging flocculation may occur. Bridging flocculation is a phenomenon that occurs when sufficient levels of strongly adsorbing polymer is added to a latex dispersion ${ }^{17,20}$. 
Bridging is when polymer chains of high molecular weight connect multiple particles together through simultaneous adsorption. Depending on the concentrations and natures of the surfactants, thickeners, and latexes in an aqueous system, HEUR bridges can form between latex surfaces, surfactant micelles, flower-like micelles, and even mixed micelles ${ }^{12,21,29}$. Bridging flocculation leads to clusters of particles known as flocs. These flocs are able to collide and merge with other large flocs in suspension until they are of sufficient size to fall and sediment ${ }^{21}$.

One method of bridging flocculation is in the case of strongly adsorbing polymers of sufficient length to bridge directly between two particles. An example schematic can be seen in Figure 9. This is known as direct bridging and is only likely to occur in systems where latex particle surface coverage is $l o w^{36}$. In this case, depending on the affinity to the surface, bridges form, break, and re-form constantly ${ }^{37}$.
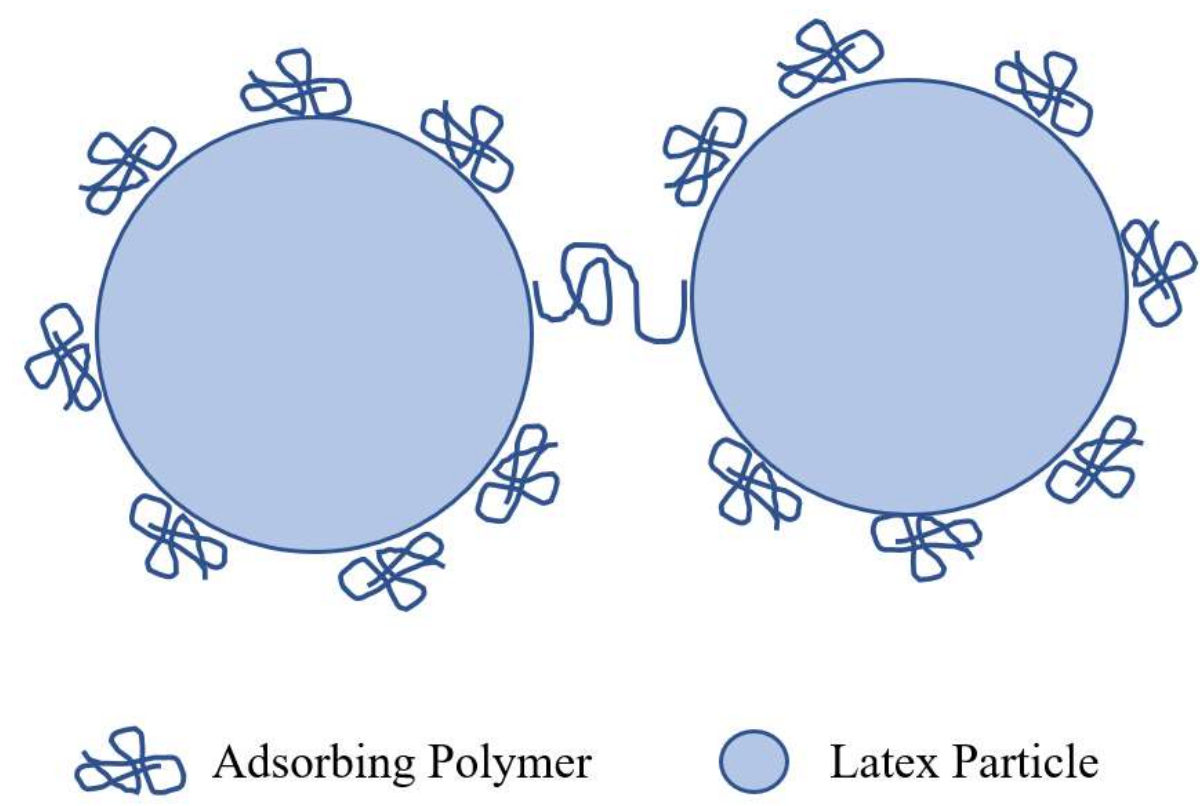

Figure 9. Bridging Flocculation by Direct Bridging Mechanism 
The second form of bridging that is able to occur is known as indirect bridging. This phenomenon is seen at much higher concentrations of associative thickeners. Instead of the associative thickeners directly bridging between two latex particles, thickener chains can interact and bridge between flower micelles and latex particles ${ }^{36}$. Networked systems like these become much more elastic and have much longer bridges between latex particles. These bridging networks only become more complex with the addition of surfactants $^{21}$.
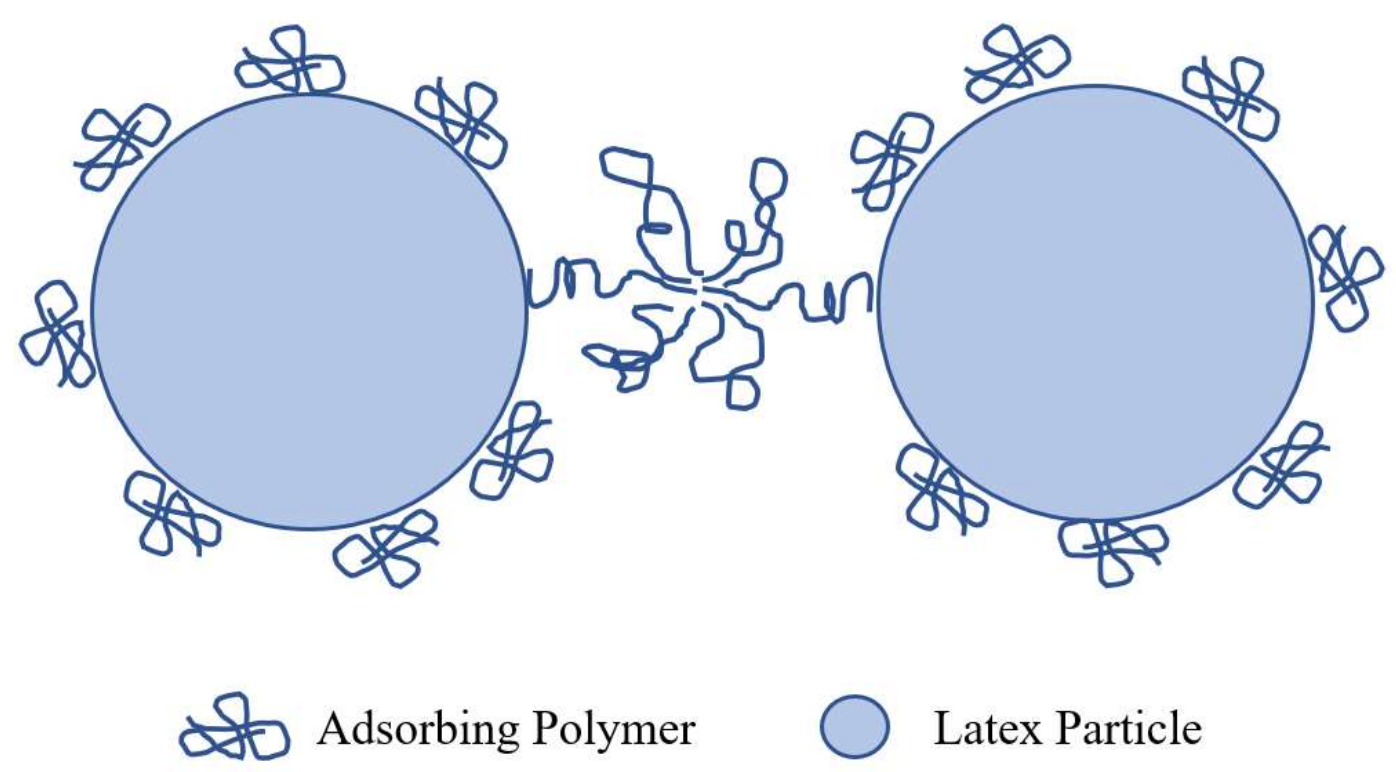

Figure 10. Bridging Flocculation by Indirect Bridging Mechanism 


\subsection{Role and Effects of Surfactants}

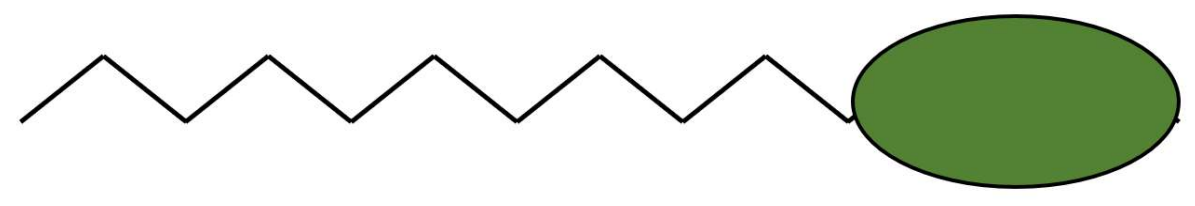

Figure 11. Schematic of a generic surfactant molecule demonstrating the hydrophobic tail and the hydrophilic head (in green)

Surface active agents, referred to as surfactants, are a class of molecules that lower interfacial tension between two phases whether it be solid-liquid, gas-liquid, or even liquid-liquid $^{6,17}$. Surfactants play an important role in latex-based paints as they are used at all stages starting from the synthesis and stabilization of the latex particles themselves to the modification of the properties of the final paint formulation. Depending on the type and classification of surfactant it will have different properties and functions. As can be seen in Figure 11, surfactants are amphiphilic compounds that have a head and a tail. Generally, the head is hydrophilic or water-loving while the tails are hydrophobic or oil-loving ${ }^{3,6,17}$. In some cases, surfactants can have complex structures with multiple hydrophobic tails or hydrophilic sections but for the scope of this work only simple single head-tail surfactants will be explored. Surfactants are commonly classified by the nature of the surfactant head. As seen in Figure 12, there are nonionic species, anionic, cationic, and zwitterionic species that respectively have heads with no charge, negative charge, positive charge, and regions of both charges ${ }^{6,17,20}$. 
a)

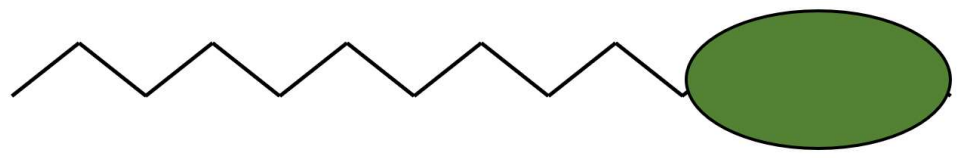

b)

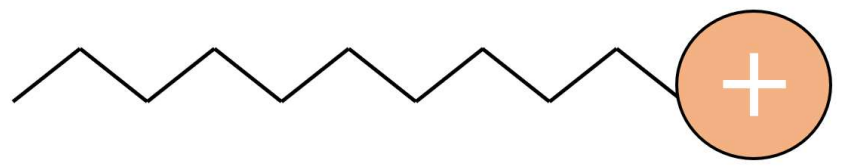

c)

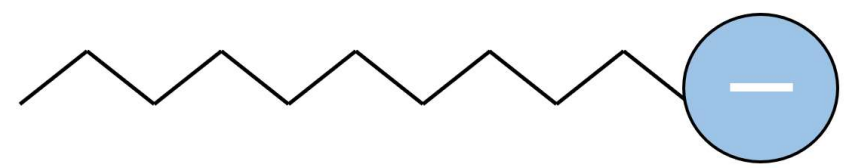

d)

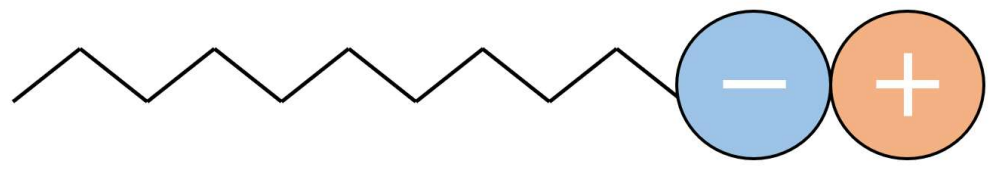

Figure 12. The 4 major types of surfactant as categorized by head type: a) nonionic, b) cationic, c) anionic, d) zwitterionic

When a small amount of surfactant is added to HEUR-thickened latex systems, mixed micelles and complex network structures are able to form ${ }^{26}$. With the introduction of these mixed micelles, more active HEUR bridges can be active at a given time. A viscosity increase is seen until a critical surfactant concentration, at which point viscosity decreases due to...

\subsection{Relevant Research and Statement of Intent}

With all these ingredients and their effects on stability and flow behavior, it is important to consider how these mechanisms cumulatively work. Aspects of colloidal stability, viscoelastic dashpot and spring systems, micellar networks, ionic strength and others all must be considered when predicting properties in more complex systems. Quaternary systems of water, latex particles, surfactant, and associative thickener have unique stability and flow behaviors depending on both the properties and concentrations of each ingredient. In previously published work, plots known as dispersion phase 
diagrams were created for aqueous Latex-HEUR-Surfactant systems to determine the concentration ranges for stable and functional dispersions ${ }^{29}$. In this work it was found that at low concentrations of HEUR and surfactant, bridging flocculation dominated. Then with an increase in either surfactant or HEUR, a stable region could be found (the white regions in Figure 13). However, with an excessive increase in either HEUR or SDS, depletion flocculation would dominate, making the system unstable leading to syneresis or phase separation ${ }^{29}$.
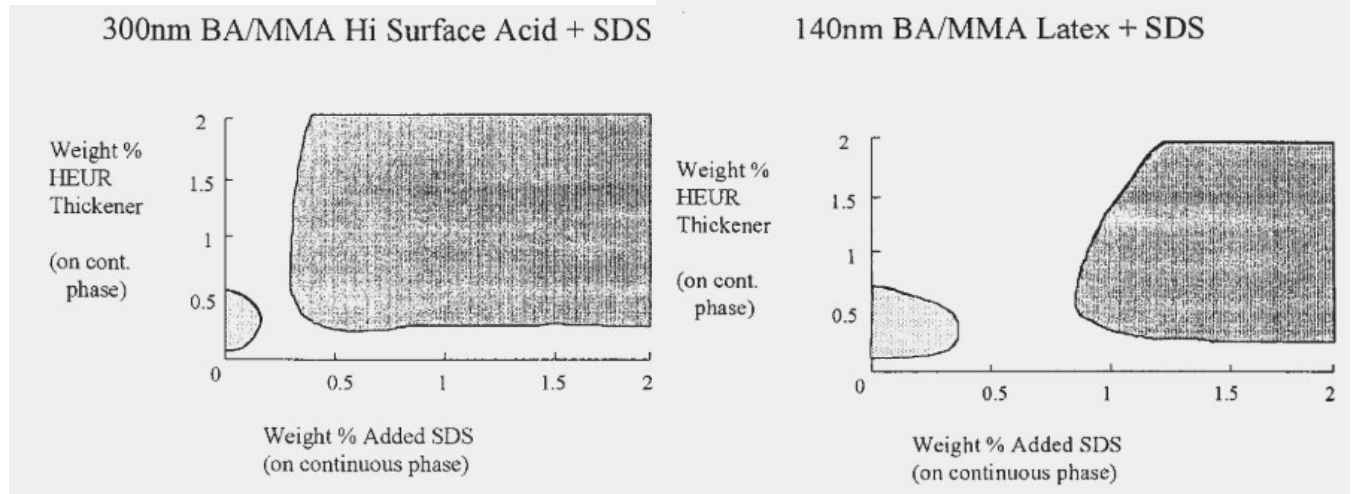

Figure 13. Dispersion phase diagrams of two BA/MMA-HEUR-SDS dispersions ${ }^{29}$

Previous work performed by the research team that this current body of work stems from not only includes exploration into dispersion phase diagrams but also the rise of unique flow behavior. Throughout the works, a series of commercial products as well as experimental materials have been used to further explore stability and rheological behavior of quaternary systems. Staying consistent with work by Edward Kostansek, Yasufumi Otsubo, these works have maintained latex loading at approximately 25 volume $\%^{29,37,38}$. This level of latex particle loading has been used as it is a sufficient level to better simulate the packing or crowding of a more fully formulated real world 
paint system ${ }^{13}$. Additionally noted in the work by Kostansek, they used $300 \mathrm{~nm}$ diameter latex particles as a sort of standard size, but it was also found that with decreases in latex particle size comes significantly increased surface area and a larger stable region in the dispersion phase diagram ${ }^{1,12}$.

In further work performed by Travis Smith, preceding this work, a concentration range and molecular weight of HEUR associative thickener was found to display unique flow behavior and stability. With lower molecular weight experimental HEUR associative thickeners, ranging from $9400-18,800 \mathrm{~g} / \mathrm{mol}$, phase separation was prominent ${ }^{11}$. With an increase in molecular weight to a nominal 35,000 M.W. PEGbackbone HEUR there was a rise in stability and distinct flow regimes. Specifically, there was the presence of Newtonian plateaus at low shear rates that led into unique shearthickening peaks in the thickener concentration range of 0.20 and $0.75 \mathrm{wt} . \% 5,13$. This work had a primary focus on working with well-characterized experimental materials instead of common commercial products, providing further insight into the mechanics at play. For the scope of this work, the materials and methods were chosen to further explore these experimental materials, their interactions, and their unique stability and flow behavior.

\section{MATERIALS AND METHODS}

\subsection{Materials}

The thickener for this series of experiments was one of a series of experimental batches of well-characterized HEUR thickeners. The primary thickener tested in this work was a HEUR with 795 ethylene oxide units capped with 18 carbon length alkyl terminal ends. This will be referred to as C18-EO795 and can be seen in Figure 14. The 
number average molecular weight of C18-EO795 is $26.8 \mathrm{~kg} \mathrm{~mol}^{-1}$. Despite the molecular weight being below $35 \mathrm{~kg} \mathrm{~mol}^{-1}$, the nomenclature will remain as C18-EO795 for clarity and consistency with previous work performed by this research group ${ }^{5,13}$.

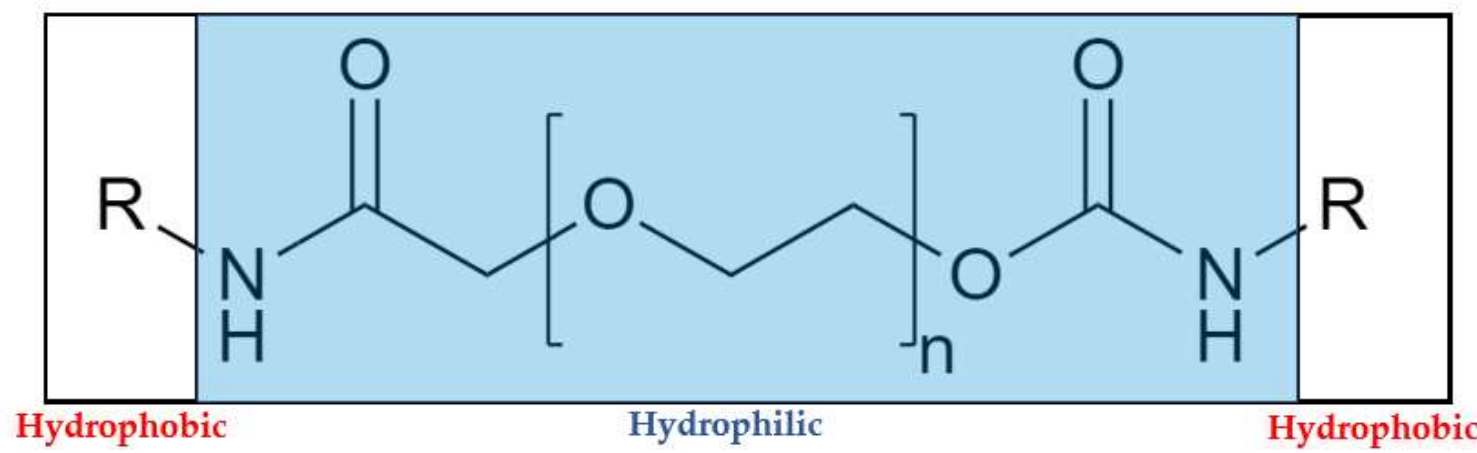

Figure 14. Structure of C18-EO795 HEUR Thickener. [n= 795, $\mathrm{R}=\mathrm{C} 18$, Mn of $26.8 \mathrm{~kg}$ mol-1.]

This experimental HEUR polymer was provided by Dow Chemical Co. This thickener was provided in the form of large, white solid chunks. Prior to addition to any samples, this solid was mixed with deionized water in a $500 \mathrm{~mL}$ glass jar to a volume of $250 \mathrm{~mL}$ at a concentration of $5 \mathrm{wt} . \%$. HEUR solids were added to deionized water and allowed to mix on a rolling mixer for 3 days at approximately 4-5 RPM until homogeneous. This stock jar method made future homogeneous mixing of the thickener into small batches much easier.

Additionally, there were two latexes that were synthesized, characterized and provided by the same Dow research team. These include a poly (butyl acrylate-co-methyl methacrylate) (BA/MMA) latex and a poly (butyl acrylate-co-styrene) (BA/STY) latex. The properties of these latexes are listed in Table 1 below 
Table 1: Experimental Latex Properties (where BA is butyl acrylate, MMA is methyl methacrylate, MAA is methacrylic acid).

\begin{tabular}{|c|c|c|}
\hline Property & BA/STY & BA/MMA \\
\hline Composition & $55 \mathrm{BA} / 43$ STY/2 MAA & 55 BA/43 MMA/2 MAA \\
\hline Solids (wt\%) & 45 & 45 \\
\hline Glass Transition $\left({ }^{\circ} \mathbf{C}\right)$ & 14 & 10 \\
\hline Particle Diameter $(\mathbf{n m})$ & 123 & 126 \\
\hline $\mathbf{p K a}$ & 8.5 & 8.3 \\
\hline $\mathbf{p H}$ & 9.6 & 9.3 \\
\hline
\end{tabular}

A total of 6 surfactants were used throughout this set of experiments. Three of these surfactants were nonionic and three of them were anionic. The nonionic surfactants included Triton X-100 (Dow Chemical), Tergitol 15-S-40 (Dow Chemical), and TSP-16 (Stepan Co.). The anionic surfactants included sodium dodecyl sulfate (Fisher Scientific), TSP-16S (Stepan Co.), and TSP-16PE30 (Stepan Co.). Ammonium hydroxide was used for samples prepared with TSP-16PE30 to adjust $\mathrm{pH}$ to levels above 8.5. All surfactant materials were used as provided with no additional modifications. Structures and additional information regarding the surfactants used in this work can be seen in Figure 15. 
a)

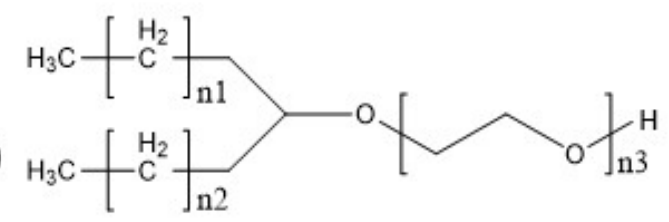<smiles>O=S(=O)([O-])OCCOc1c(/C=C/c2ccccc2)cc(/C=C/c2ccccc2)cc1/C=C/c1ccccc1</smiles>

b)<smiles>COc1ccc(C(C)(C)CC(C)(C)C)cc1</smiles><smiles>c1ccccc1</smiles>

e)<smiles>O=C(O)COc1c(/C=C/c2ccccc2)cc(/C=C/c2ccccc2)cc1/C=C/c1ccccc1</smiles>

f)

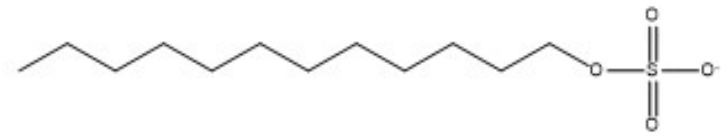

Figure 15. a) Tergitol 15-S-40 [n1+n2=11-15 and $\mathrm{n} 3=41]$; b) Triton $\mathrm{X}-100$ [n 9.5]; c) TSP-16 [n=16]; d) TSP-16S [n=16]; e) TSP-16PE30 [n=16, pH:0.9]; f) Sodium dodecyl sulfate. 
Table 2. Approximate molecular weight and critical micelle concentration values of the 6 surfactants.

\begin{tabular}{|l|c|c|c|}
\hline Surfactant & M.W. (g/mol) & CMC (ppm) & CMC (mmol/L) \\
\hline SDS & 288 & $2322 \mathrm{ppm}$ & $8.1 \mathrm{mM}$ \\
\hline Tergitol 15-S-40 & $\sim 2000-2100$ & $1314 \mathrm{ppm}$ & $0.64 \mathrm{mM}$ \\
\hline Triton X-100 & 624 & $189 \mathrm{ppm}$ & $0.30 \mathrm{mM}$ \\
\hline TSP-16 & 1100 & $4.9 \mathrm{ppm}$ & $0.0045 \mathrm{mM}$ \\
\hline TSP-16S & 1200 & $50 \mathrm{ppm}$ & $0.042 \mathrm{mM}$ \\
\hline TSP-16PE30 & 1200 & $100 \mathrm{ppm}$ & $0.083 \mathrm{mM}$ \\
\hline
\end{tabular}

\subsection{Sample Preparation}

A series of latex/HEUR/surfactant samples were prepared in two different series with each focused on the different latexes, BA/MMA and BA/STY. For all samples, the latex concentration was set at 25 vol. \% for all samples. HEUR concentration was held at 0.5 wt.\% for all samples. All samples with additional surfactant start at a concentration that well exceeds the listed CMC's listed in Table_. The concentrations of surfactant were made starting at $0.0 \mathrm{wt} . \%$ as a control, up to $0.1 \mathrm{wt} . \%$, up to $0.25 \mathrm{wt} . \%$ and then in increments of $0.25(0.5,0.75,1.0 \ldots)$ until the sample appeared Newtonian in flow behavior. This method was used for both latex series and for all 6 surfactants.

Samples were prepared by adding thickener and then adding deionized water into a plastic $100 \mathrm{~mL}$ Thinky Cup. This would be hand-stirred for 30 seconds with a wooden stirring stick prior to mixing for 1 minute in a Thinky Mixer at 1000 RPM. After the thickener was mixed evenly into the water, surfactant would be added by disposable pipette to the desired weight. Finally, latex was added by pipette with occasional stirring 
into the Thinky Cup. After all ingredients were placed into the cup, the mixture was mixed for another full minute in the Thinky Mixer. Sample mixtures were then transferred to 11-dram vials and allowed to equilibrate for 6-8 days to check for any potential phase separation or visible destabilization. After the 6 to 8 -day period of equilibration, samples were lightly mixed by spatula for 2 minutes and $\mathrm{pH}$ measurements were taken with a Fisher Scientific AR-10 pH Meter. After acquisition of the equilibrated $\mathrm{pH}$, rheological tests were performed on small aliquots of the stirred mixtures.

\subsection{Rheological Testing Parameters}

The device used for all rheological testing was a TA Instruments Discovery HR-2 Hybrid Rheometer. A total of four major tests were performed on each sample, including a logarithmic flow sweep, a logarithmic amplitude sweep, a linear amplitude sweep, and a frequency sweep. Prior to these steps a flow peak hold step at a shear rate of $5.0 \mathrm{~s}^{-1}$ was added for a 2-minute period.

Table 3. Parameters for Flow Peak Hold of Waterborne Latex/HEUR/Surfactant Mixtures.

\begin{tabular}{lcc}
\multicolumn{2}{c}{ Step 1: Flow Peak Hold } \\
Parameter & Value & Units \\
\hline Temperature & 25 & ${ }^{\circ} \mathrm{C}$ \\
Duration & 120 & $\mathrm{~s}$ \\
Shear Rate & 5 & $\mathrm{~s}^{-1}$ \\
\hline
\end{tabular}


Table 4. Parameters for Flow Sweep of Waterborne Latex/HEUR/Surfactant Mixtures.

\begin{tabular}{lcc}
\multicolumn{3}{c}{ Step 2: Flow Sweep } \\
Parameter & Value & Units \\
\hline Temperature & 25 & ${ }^{\circ} \mathrm{C}$ \\
Shear Rate & $0.001-1000$ & $\mathrm{~s}^{-1}$ \\
Points per decade & 10 & \\
Steady state sensing & No & \\
Equilibration time & 45 & $\mathrm{~s}$ \\
Sample time & 15 & $\mathrm{~s}$ \\
\hline
\end{tabular}

Table 5. Parameters for Logarithmic Amplitude Sweep of Waterborne Latex/HEUR/Surfactant Mixtures.

Step 3: Logarithmic Amplitude Sweep

\begin{tabular}{lcc} 
Parameter & Value & Units \\
\hline Temperature & 25 & ${ }^{\circ} \mathrm{C}$ \\
Frequency & 1 & $\mathrm{~Hz}$ \\
Strain $\%$ & $0.01-100$ & $\%$ \\
Points per decade & 5 & \\
Conditioning time & 3 & $\mathrm{~s}$ \\
Sensing time & 3 & $\mathrm{~s}$ \\
\hline
\end{tabular}

Table 6. Parameters for Linear Amplitude Sweep of Waterborne Latex/HEUR/Surfactant Mixtures.

\begin{tabular}{lcc}
\multicolumn{3}{c}{ Step 4: Linear Amplitude Sweep } \\
Parameter & Value & Units \\
\hline Temperature & 25 & ${ }^{\circ} \mathrm{C}$ \\
Frequency & 1 & $\mathrm{~Hz}$ \\
Strain \% & $2-100$ & $\%$ \\
Increment & 2.0 & $\%$ \\
Conditioning time & 3 & $\mathrm{~s}$ \\
Sensing time & 3 & $\mathrm{~S}$ \\
\hline
\end{tabular}


Table 7. Parameters for Frequency Sweep of Waterborne Latex/HEUR/Surfactant Mixtures.

\begin{tabular}{lcc}
\multicolumn{2}{c}{ Step 5: Frequency Sweep } & \\
Parameter & Value & Units \\
\hline Temperature & 25 & ${ }^{\circ} \mathrm{C}$ \\
Frequency & $0.01-100$ & $\mathrm{~Hz}$ \\
Strain \% & 0.1 & $\%$ \\
Points per decade & 5 & \\
Conditioning time & 3 & $\mathrm{~s}$ \\
Sensing time & 3 & $\mathrm{~s}$ \\
\hline
\end{tabular}

The flow sweeps were performed across a range of shear rates ranging from 0.001 to $1000 \mathrm{~s}^{-1}$. A total of 10 points per logarithmic decade were collected. An equilibration time of 45 seconds was used in conjunction with a 15 second averaging time. The logarithmic amplitude sweep was performed at a frequency of $1.0 \mathrm{~Hz}$ with the strain percent ranging from 0.01 to $100 \%$. A total of 5 data points was collected for each logarithmic decade. Both the conditioning and sampling time were set to be 3.0 seconds each. The linear amplitude sweep was performed at a frequency of $1 \mathrm{~Hz}$ ranging from 2.0 to $100 \%$ strain at an increment of $2 \%$. Again, the conditioning and sampling time were both 3.0 seconds. The final test performed on a sample was the frequency sweep. The frequency sweep was performed at a strain percent of $0.1 \%$ across a range of 0.01 to 100.0 Hz. For each decade, there were 5 data points collected. Conditioning and sampling times were set to 3.0 seconds for all frequency sweeps as well. All trials were held at $25.0^{\circ} \mathrm{C}$ by a water-cooled base plate system. 


\section{RESULTS AND DISCUSSION}

The results of this body of work are going to be organized in a manner that is conducive to the understanding of the mechanisms and phenomena. Initially, vocabulary and generic information will be provided to make analysis more concise and accurate. The first set of results to be analyzed are the viscosity flow plots. These plots demonstrate the same behavior as work previously published in this research group using the same BA/STY latex. The flow plots will be organized starting with BA/STY, followed by BA/MMA systems, then a final comparative analysis. Beyond flow plots, dynamic analysis will be separated into two sections, one for each latex. Each sample set will have results from both the amplitude sweeps and frequency sweeps.

\subsection{Flow Sweep Analysis of BA/STY-Surfactant-Thickener Aqueous Systems}

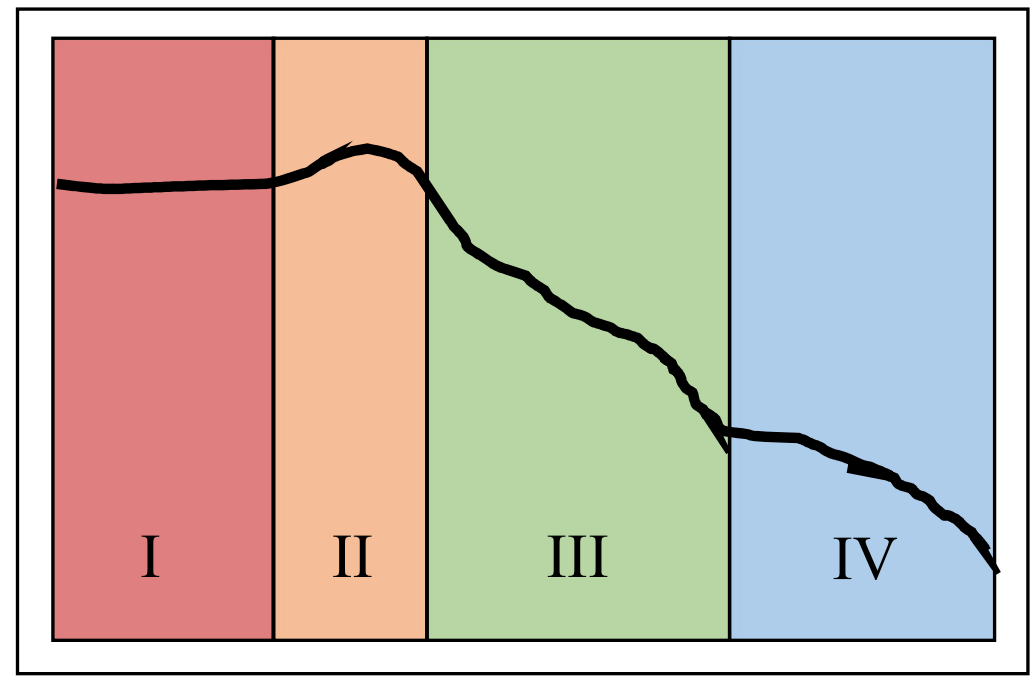

Figure 16. Generic flow sweep to demonstrate the four major regimes.

It is important to establish the vocabulary that will be used to refer to these viscosity plots. As seen in Figure 16, there are 4 major regimes that are seen across all of the flow sweeps. At low to mid-level surfactant concentrations, all systems demonstrate 
four distinct regimes. At higher levels of surfactant concentrations, the clear boundaries between these regimes disappear as viscosity decreases and samples demonstrate highly Newtonian behavior.

Regime I includes the region before the shear-thickening maximum and will be referred to as the Newtonian plateau. Regime II will specifically refer to the shearthickening maximum. Regime III will refer to the shear-thinning region after the shearthickening maximum but before the highest shear rates. Regime IV will be referred to as the high shear rate region, which generally lies between $10^{1}-10^{3} \mathrm{~s}^{-1}$. This division of the flow sweeps into regimes will allow for simple but thorough analysis of trends at each shear rate range. 


\subsubsection{BA/STY-HEUR Systems with $0.1 \mathrm{wt} \%$ Additional Surfactant}

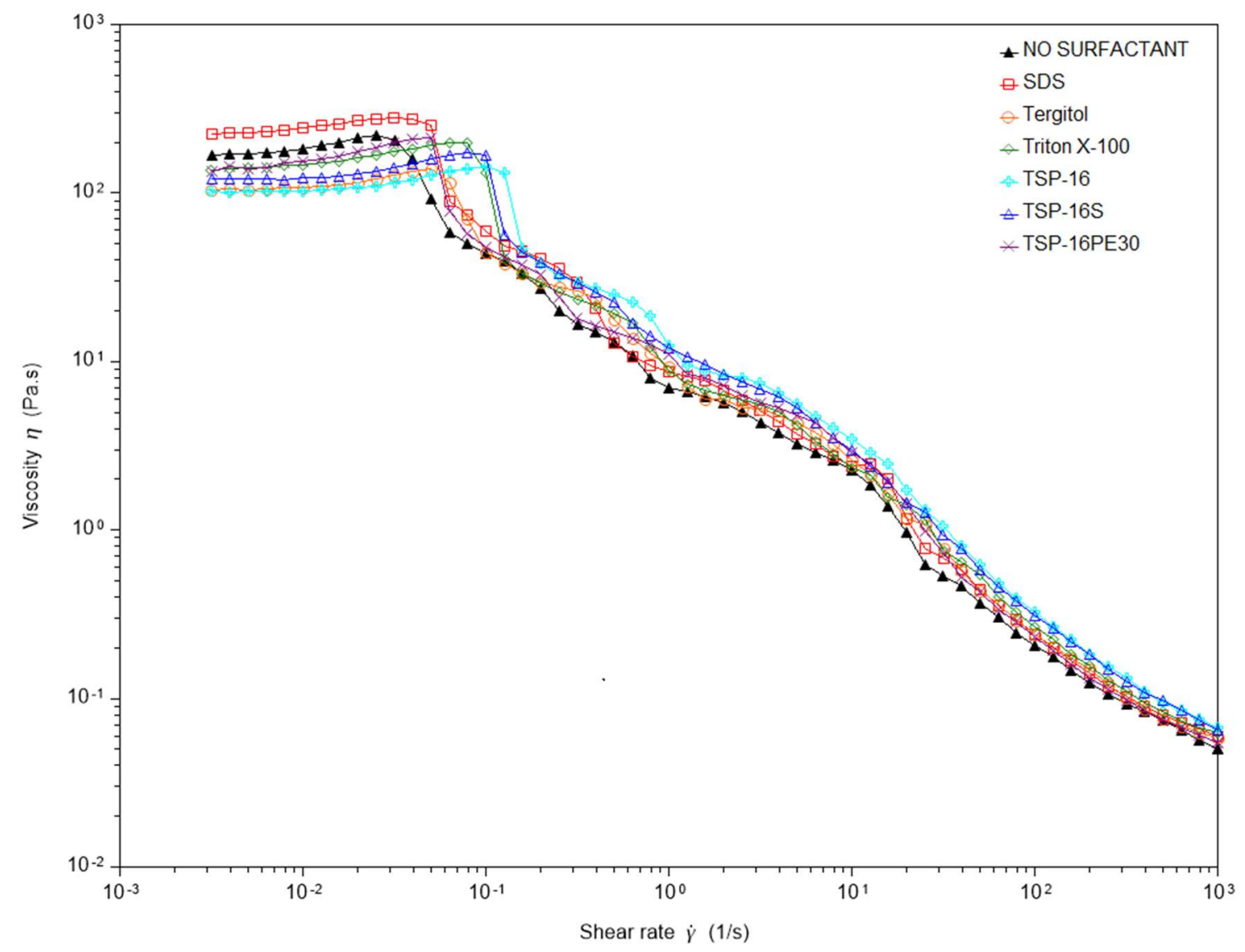

Figure 17. Flow Sweep Overlay of BA/STY Latex at 25 vol. \%, C18-EO795 thickener at $0.5 \mathrm{wt} \%$, Surfactant at $0.1 \mathrm{wt} . \%$ systems.

When introducing a low concentration of additional surfactant to the system, it can be seen in Figure 17, that the overall trend for five of the surfactant systems is a decrease in viscosity in Regimes I and II. The exception to this initial trend is SDS. Sodium Dodecyl Sulfate has been seen in previous literature to perform complexation with PEG chains, leading to a hydrodynamic volume increase as the interaction between PEG and SDS disrupts hydrophobic hydration ${ }^{39}$ and leads to micelle structure shifting to a shape between spherical and rod-like ${ }^{40}$. Given that the chosen HEUR thickener has a 
PEG backbone, the increase in viscosity at low shear rates can likely be attributed to this association of SDS with the C18-EO795 chain.

The major trend to note is the shift of the shear-thickening maximums downward and rightward. In the case of 5 surfactants, the shear-thickening maximums shift to lower viscosities and to higher shear rates, excluding SDS. The shift to lower viscosities can be attributed to the competitive interference of the surfactant on the surface of the latex particles. As more surfactant is introduced, surfactant molecules are able to occupy association sites on the latex surface. The surfactant hydrophobes likely have a higher affinity than the associative ends of the $\mathrm{C} 18$-EO795 chain. As this occurs, the overall number of active bridges formed at any given time by the associative thickener molecules is decreased. A decrease in active bridges will in most cases lead to a decrease in viscosity when compared to the system with no additional surfactant.

The shift rightward to higher shear rates for the shear-thickening maximum relates to the time scale of the rate of association and dissociation of the HEUR transient network. The HEUR bridges in this quaternary system are not permanent and associate and dissociate at a given rate determined by the affinity of the hydrophobic ends for different regions on the surface of the latex particle. If there is a high affinity between the HEUR ends and the latex surface, then the bridges will last for a longer period of time. Additionally, with a high affinity for the latex surface, when the HEUR bridge dissociates, it rapidly re-associates ${ }^{1}$. However, when surfactant molecules are able to interact with the surface of the latex, this has an effect on the hydrophobicity of the latex particle surface. In all surfactant cases at this concentration, there is a rightward shift of the shear thickening maximum. A shift of this maximum to higher shear rates means that 
the HEUR molecules are spending less time associated to the latex surface than in the control system.

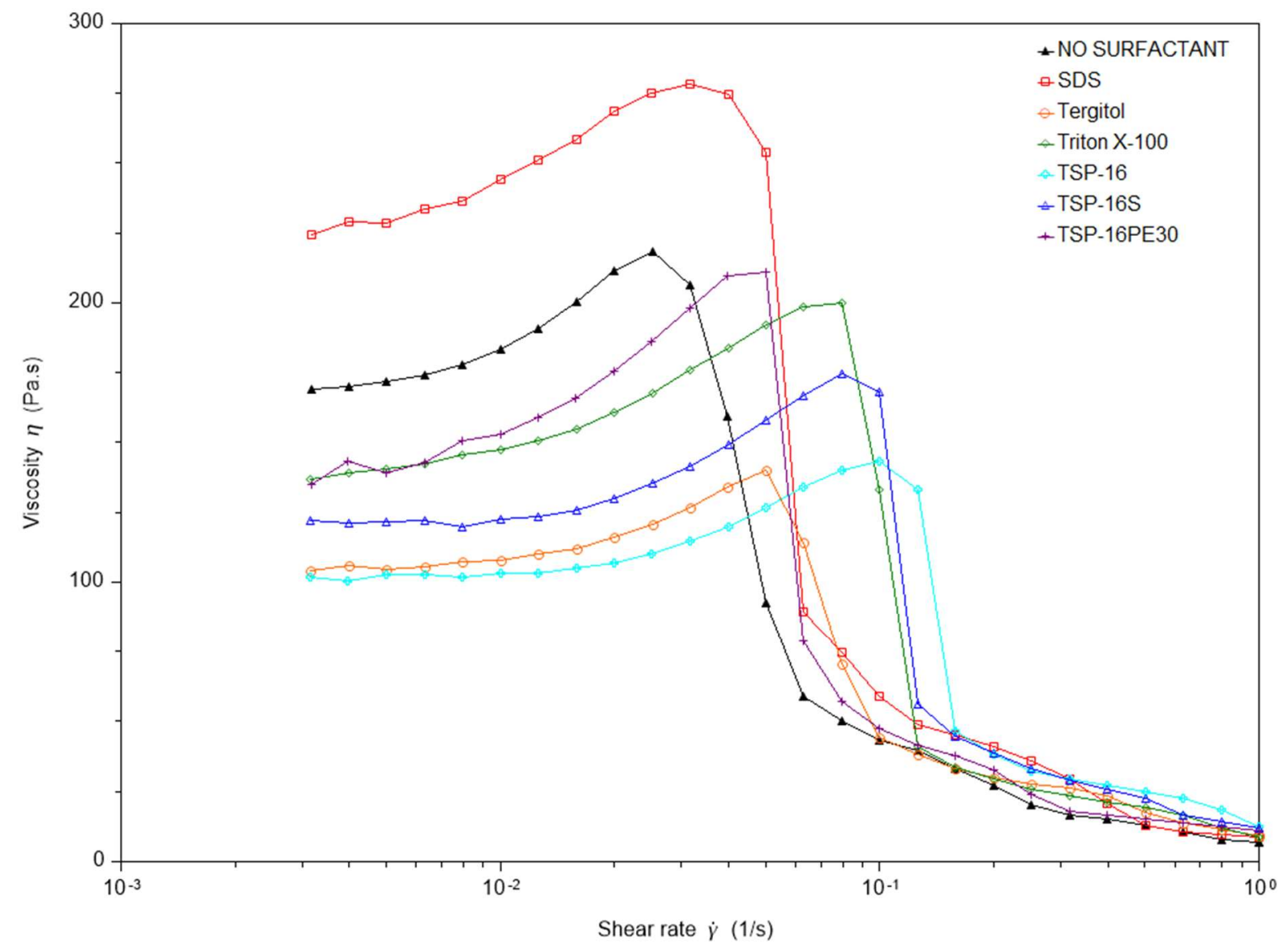

Figure 18. Regimes I and II with linear y-axis for C18-EO795/BA-STY, with $0.1 \mathrm{wt} \%$ surfactant systems.

Data from Figure17 was replotted in Figure 18 to properly demonstrate the scale and difference between samples. With Regimes I and II more clearly portrayed, it is easily seen that the nonionic surfactant TSP-16 is the rightmost shifted shear-thickening maximum at $0.010 \mathrm{~s}^{-1}$, whereas the anionic TSP-16PE30 is the leftmost maximum (excluding SDS) at $0.050 \mathrm{~s}^{-1}$. The nonionic Tergitol 15-S-40 samples show the lowest overall shear-thickening maximum at a viscosity of $140.1 \mathrm{~Pa}^{*} \mathrm{~s}$. As seen in Figure 18 
however, there is no noticeable trend regarding the ionic nature of the surfactants and the shifting of the shear-thickening maximums. The most noteworthy shifting is that of the sample with SDS to a viscosity maximum of $278.2 \mathrm{~Pa}^{*}$ s compared to the peak of the control sample at $218.3 \mathrm{~Pa}^{*} \mathrm{~s}$.

\subsubsection{BA/STY-HEUR Systems with $0.25 \mathrm{wt} \%$ Additional Surfactant}

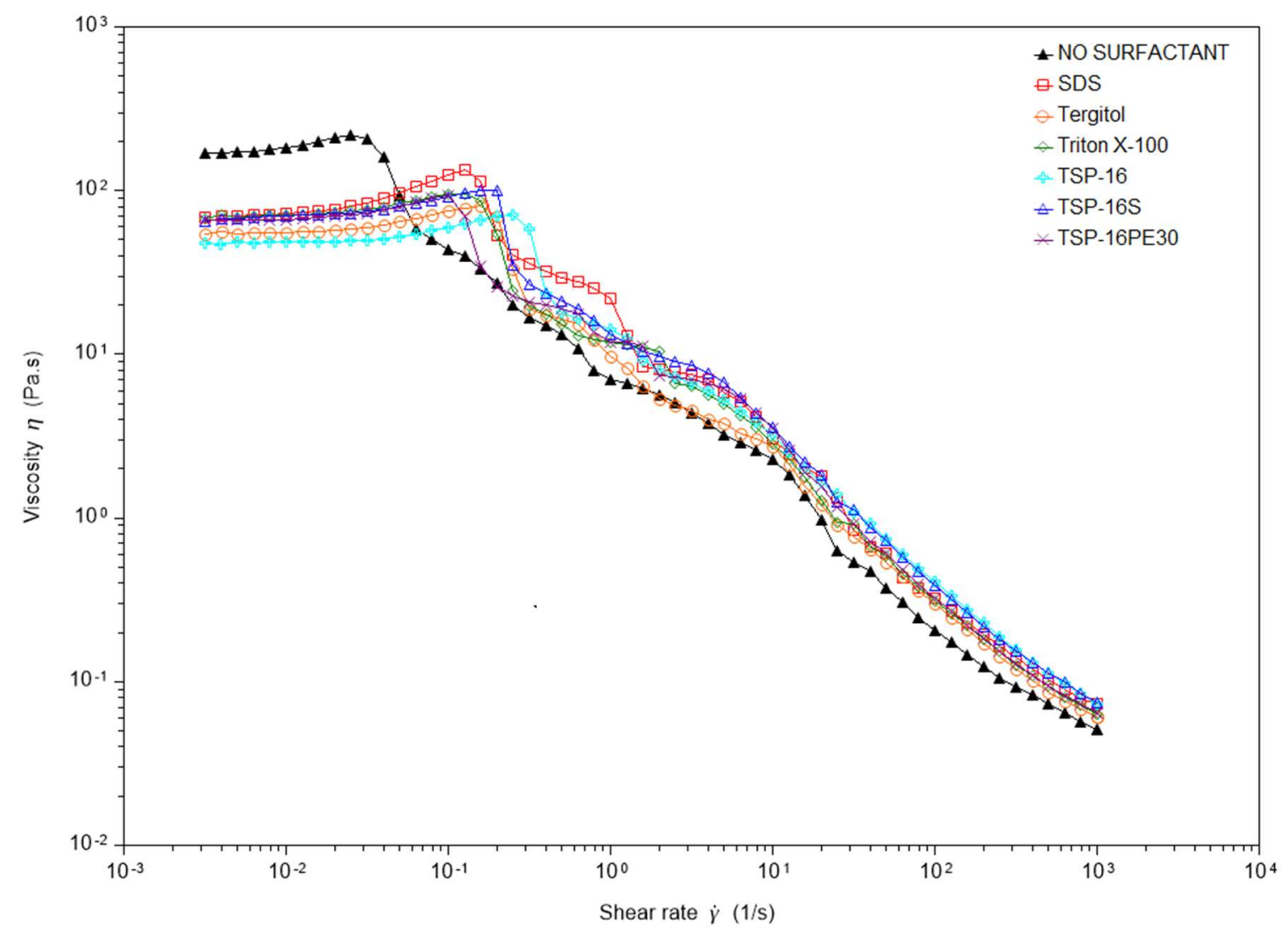

Figure 19. Flow Sweep Overlay of BA/STY Latex at 25 vol. \%, C18-EO795 thickener at $0.5 \mathrm{wt} \%$, Surfactant at $0.25 \mathrm{wt} . \%$ systems.

With an increase in surfactant levels to 0.25 wt. \% a simple decrease in Regime I and II viscosities is seen. All samples show a decrease of viscosity in the Newtonian plateau as well as the shear-thickening maximum. This decrease in viscosity is expected 
as there is more than double the amount of surfactant active in the quaternary system than the $0.1 \mathrm{wt} \%$ systems. More surfactant molecules can interact with the surface of the latex particles and reduce the overall number of active HEUR bridges. Regime III becomes more complex with a distinct multistep nature. This behavior in Regime III is something to be resolved in future work. Until this regime is fully resolved, it is not possible to identify if these are simple steps or a series of shear-thickening peaks being masked by more prominent interactions in the system. Compared to Regime III, Regime IV demonstrates a simpler shear-thinning behavior.

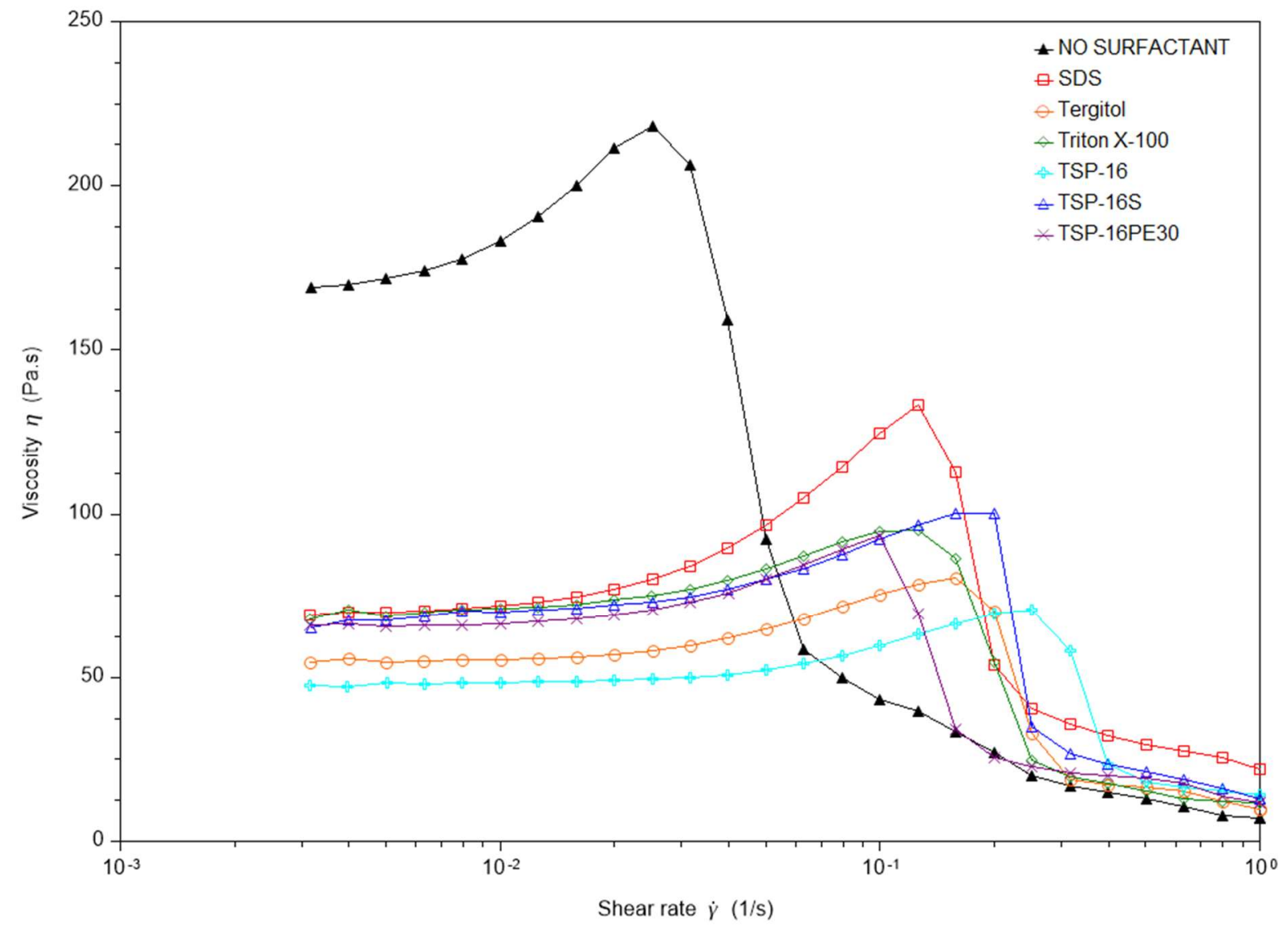

Figure 20. Regimes I and II with linear y-axis for C18-EO795/BA-STY, with $0.25 \mathrm{wt} \%$ surfactant systems. 
Figure 20 shows that the sample with TSP-16 remains the sample with the rightmost shifted shear-thickening peak, now located at $0.251 \mathrm{~s}^{-1}$. The sample with TSP16PE30 also remains as the left most of the maximums at $0.100 \mathrm{~s}^{-1}$. The sample with SDS has a shear thickening peak at a higher viscosity than all other surfactant systems at a viscosity of $133.1 \mathrm{~Pa}^{*}$ s but Regime I has dropped into the same magnitude as the other samples for its Newtonian Plateau.

\subsubsection{BA/STY-HEUR Systems with $0.50 \mathrm{wt} \%$ Additional Surfactant}

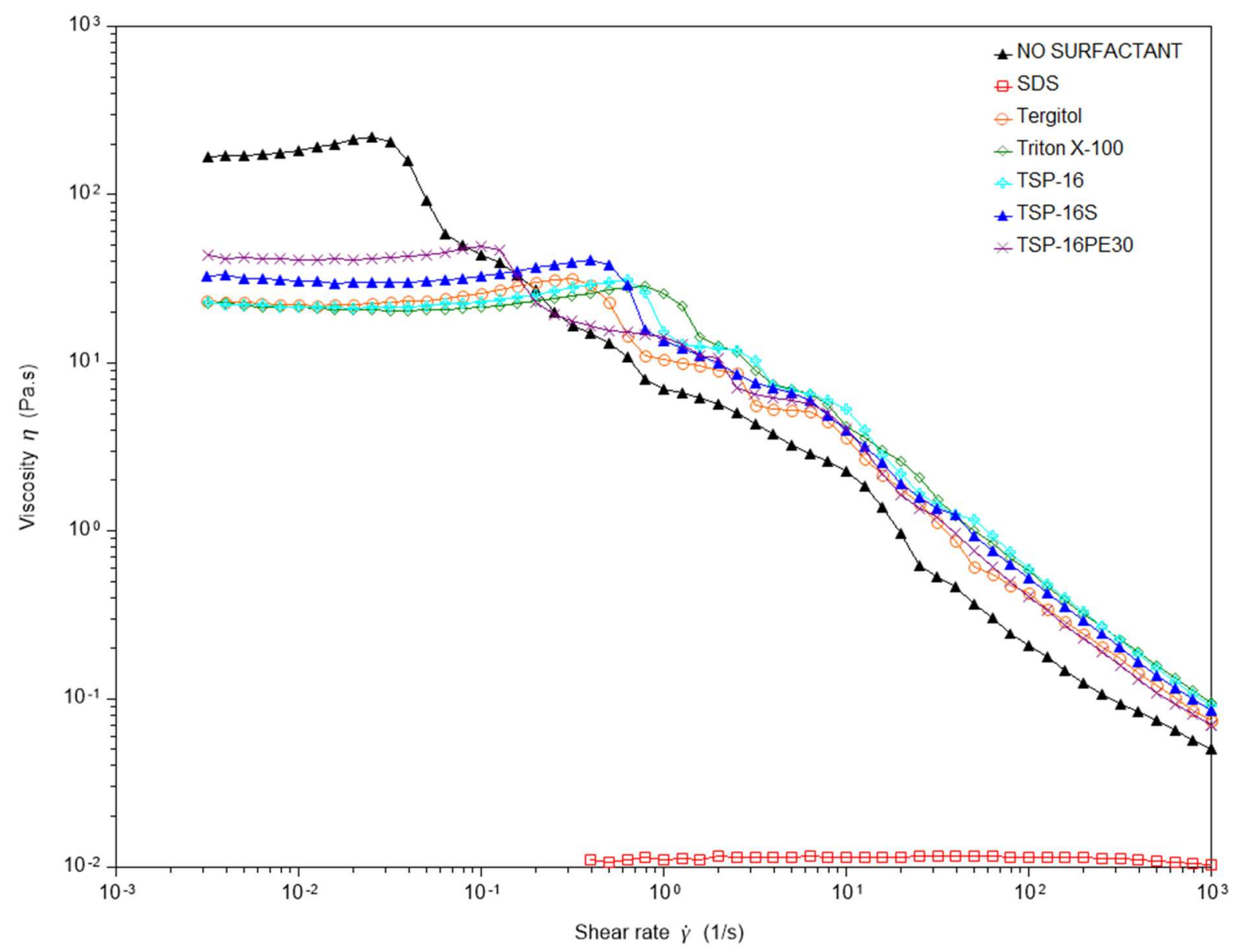

Figure 21. Flow Sweep Overlay of BA/STY Latex at 25 vol. \%, C18-EO795 thickener at $0.5 \mathrm{wt} \%$, Surfactant at $0.50 \mathrm{wt} . \%$ systems. 
Figure 21 shows the overlay for BA/STY systems with $0.50 \mathrm{wt} \%$ of additional surfactant. The sample with additional SDS shows near-Newtonian behavior at this concentration. This behavior can be attributed to behaviors found in previous literature. When SDS is introduced to systems with associative thickener with ethylene oxide backbones, there is a complexation mechanism that takes place. The backbones of associative polymers can interact and wrap around SDS micelles. It has been found, depending on the number of PEG units of polymer and examination method, there is a ratio varying from 1.9 to 5.6 of ethylene oxide units to SDS molecules ${ }^{41}$. It was also found that for a PEG 10000 chain, that length was sufficient for about one chain to wrap around an SDS micelle ${ }^{41}$. These interactions between the PEG backbones and the SDS micelles are enough to entirely disrupt the HEUR associative ends from freely associating and forming a continuous bridging network, making the viscosity drop significantly and become almost entirely Newtonian (cite synergistic paper).

At $0.25 \mathrm{wt} . \%$ of additional surfactant, it is seen that the shear-thickening maximums of all the systems have shifted to higher shear rates and lower viscosities, but in Regimes III and IV viscosities remain higher than the control. In Regimes III and IV, the entire viscosity profile appears to shift to higher shear rates when compared to the control, not just a simple increase in viscosities. 


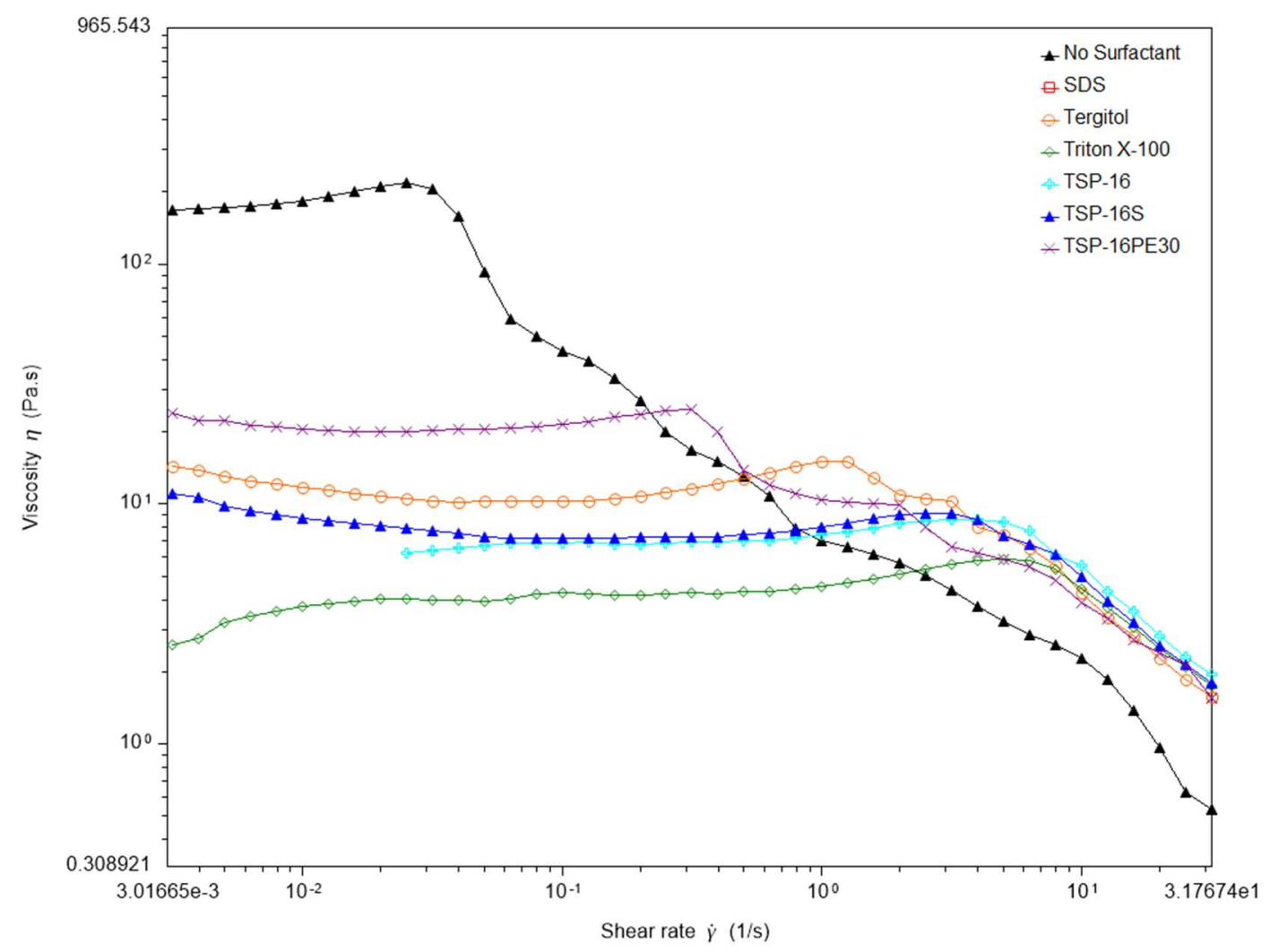

Figure 22. Zoomed-in Regimes I and II with for C18-EO795/BA-STY, with $0.25 \mathrm{wt} \%$ surfactant systems.

In Figure 22, the order of the surfactants by viscosity and shear rate can be seen. In this region, the system with Triton X-100 shows the rightward- and downward-most shifted shear-thickening peak. This is indicative of the ability of Triton X-100 to interfere with the associative thickening mechanism more effectively at this concentration than the remaining surfactant systems at higher viscosities. TSP-16PE30 appears to be the least effective at disrupting the bridging network. 
3.1.4 BA/STY-HEUR Systems with $0.75 \mathrm{wt} \%$ Additional Surfactant

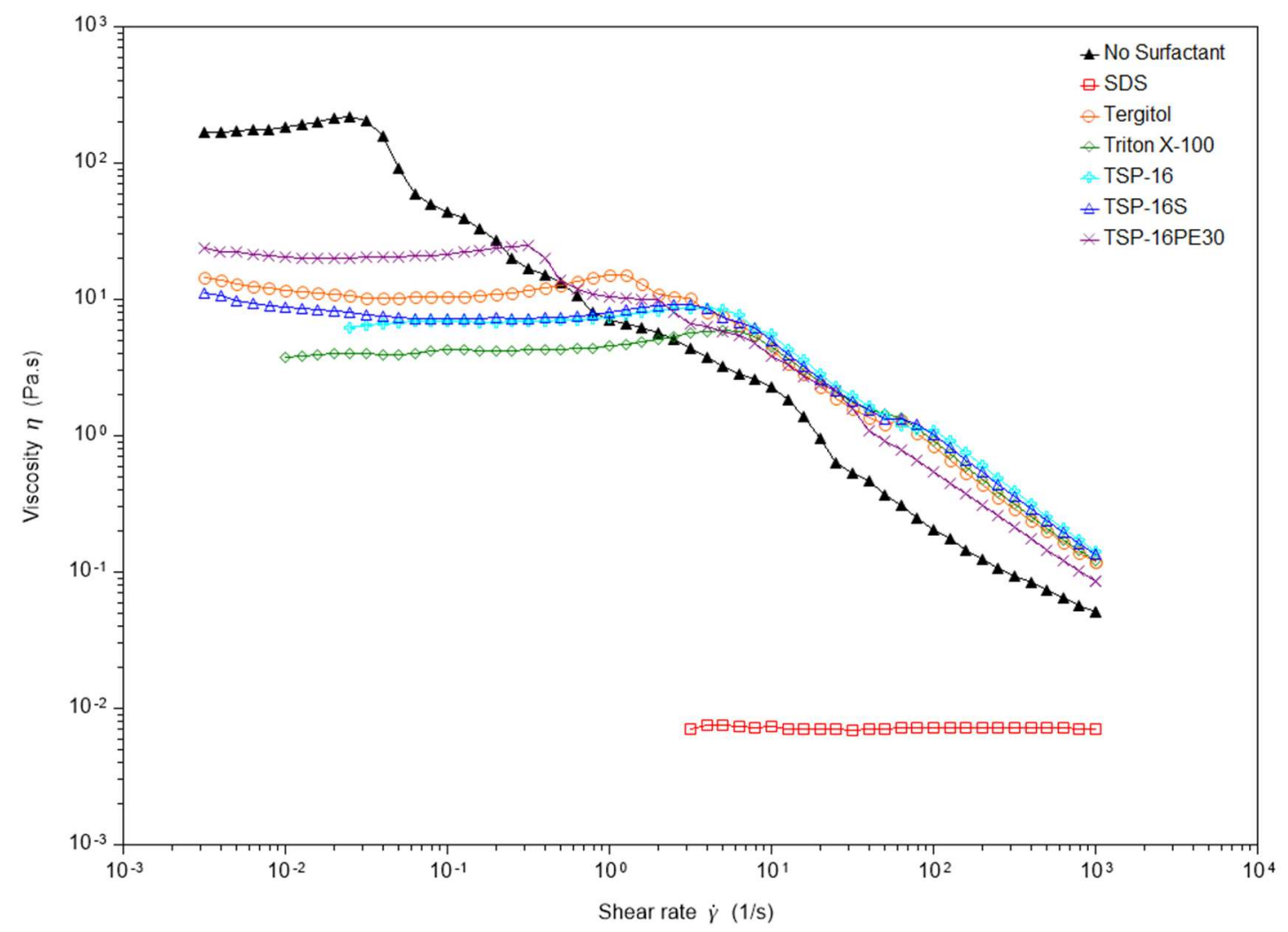

Figure 23. Flow Sweep Overlay of BA/STY Latex at 25 vol. \%, C18-EO795 thickener at 0.5 wt. $\%$, Surfactant at 0.75 wt. \% systems.

At 0.75 wt.\%, Regime I shows an extension in the Newtonian plateau for all five surfactant samples excluding SDS. There is still a rightward shifting of the shearthickening maximums as expected with the increase in concentration. The sample with SDS is displaying a complete Newtonian behavior at a viscosity similar to a system with only latex and water. Regimes III and IV for the other 5 samples show an increase in viscosity compared to the control with no surfactant around $100 \mathrm{~s}^{-1}$ where a distinct step occurs. Looking closer at Regime IV it is noticeable that TSP-16PE30 does not display the same viscosity step near $100 \mathrm{~s}^{-1}$ like the other 4 surfactants. 


\subsubsection{BA/STY-HEUR Systems with $1.0 \mathrm{wt} \%$ Additional Surfactant}

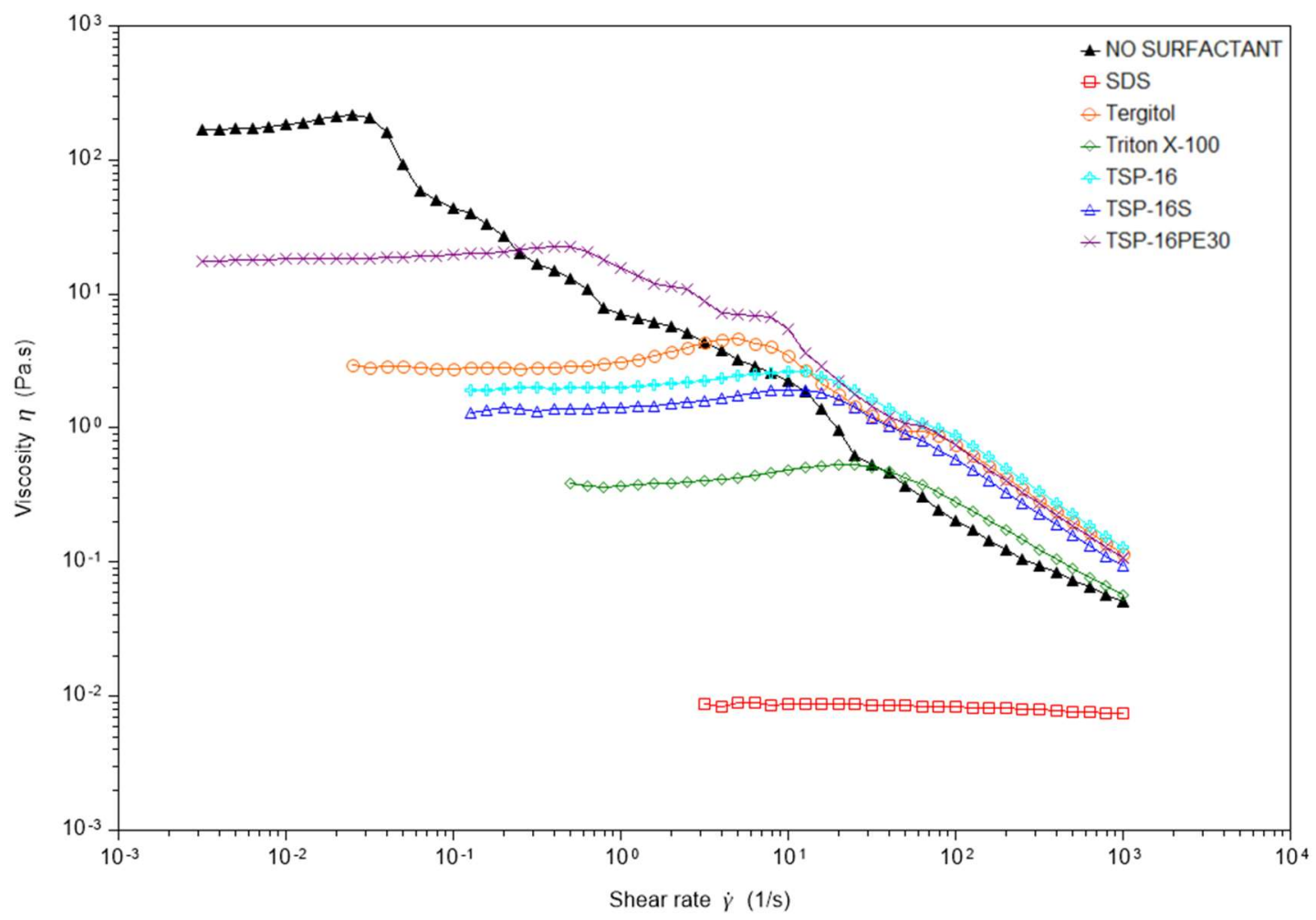

Figure 24. Flow Sweep Overlay of BA/STY Latex at 25 vol. \%, C18-EO795 thickener at 0.5 wt. $\%$, Surfactant at 1.0 wt. $\%$ systems.

Systems with 1.0 wt. \% additional surfactant can be seen in Figure 24. The trend remains that as concentration increases, the further to the right that the shear-thickening maximum shifts. Additionally, there is still an overall decrease seen in viscosity for Regimes I and II. Regimes III and IV however show an increase in viscosity over that of the control at higher shear rates. SDS continues to maintain the Newtonian behavior at this concentration and no syneresis or phase separation behavior is visible. TSP-16PE30 maintains its status as the least competitive and disruptive of the surfactants. Triton X100 has now significantly dropped in viscosity compared to the other surfactants. Based 
on the flow sweeps for the BA/STY systems, it does not appear that the ionic nature of the surfactant is as significant as the molecular weight or micelle size may be.

\subsubsection{High shear viscosity analysis of BA/STY systems}

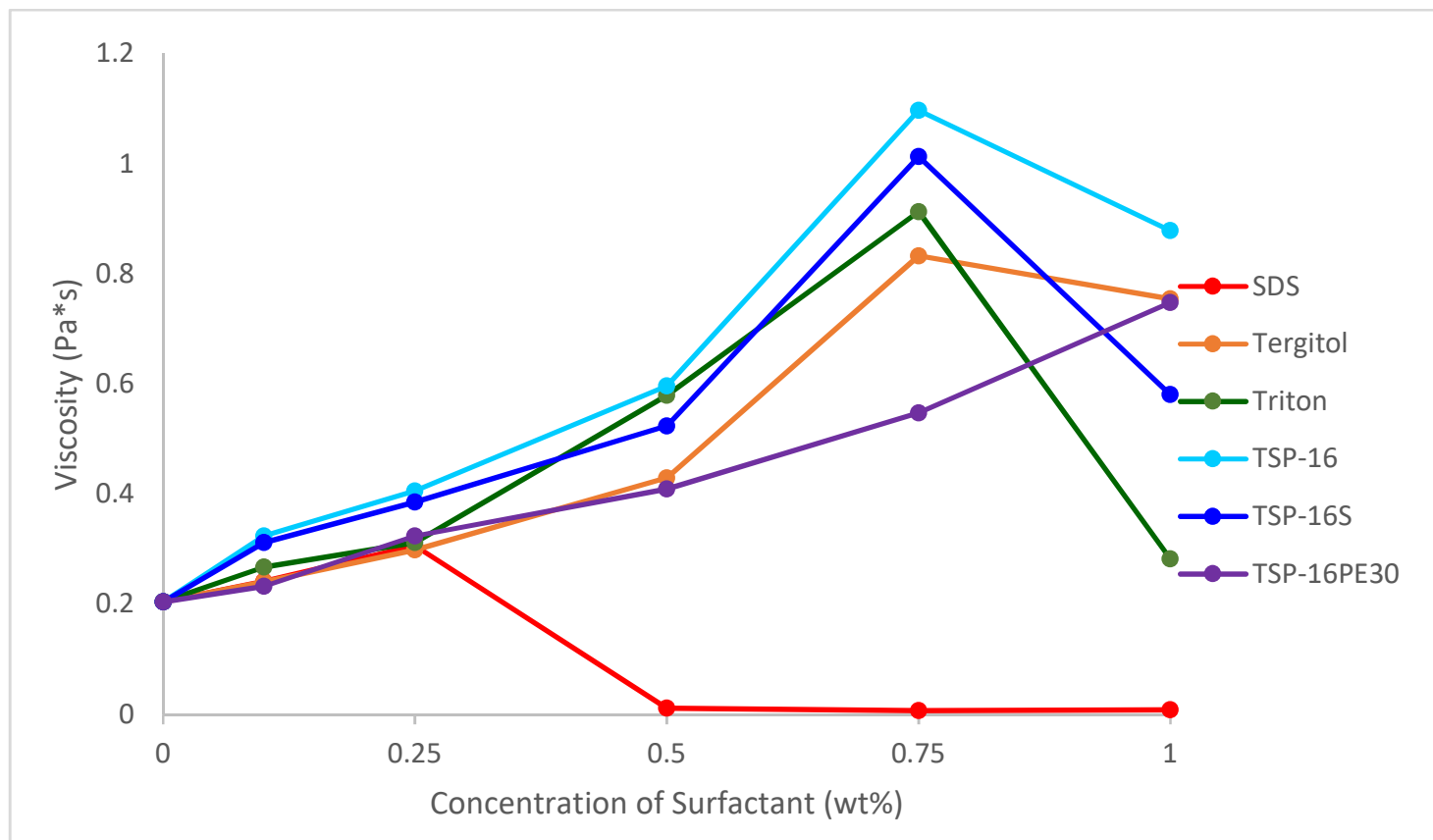

Figure 25. Viscosity values at $100 \mathrm{~s}-1$ as a function of surfactant concentration (wt. \%) for BA/STY systems

The final piece of flow sweep analysis is the behavior at high shear rates. In Regime IV from 100 to $1000 \mathrm{~s}^{-1}$, there was an interesting almost parabolic nature to the viscosities. These shear rates were chosen as the beginning and end to the simple shearthinning region. In Figures 25 and 26, there are clear peaks for five out of the six surfactants. As to be expected, SDS shows its peak and drop at the lowest concentration. Four surfactants show a peak at $0.75 \mathrm{wt}$ \%. Also, TSP-16PE30 at both 100 and $1000 \mathrm{~s}^{-1}$, does not show a peak within this concentration range so further testing would need to be 
performed at increments between 1.0 and $2.0 \mathrm{wt}$. \% of surfactant loading to potentially find this peak.

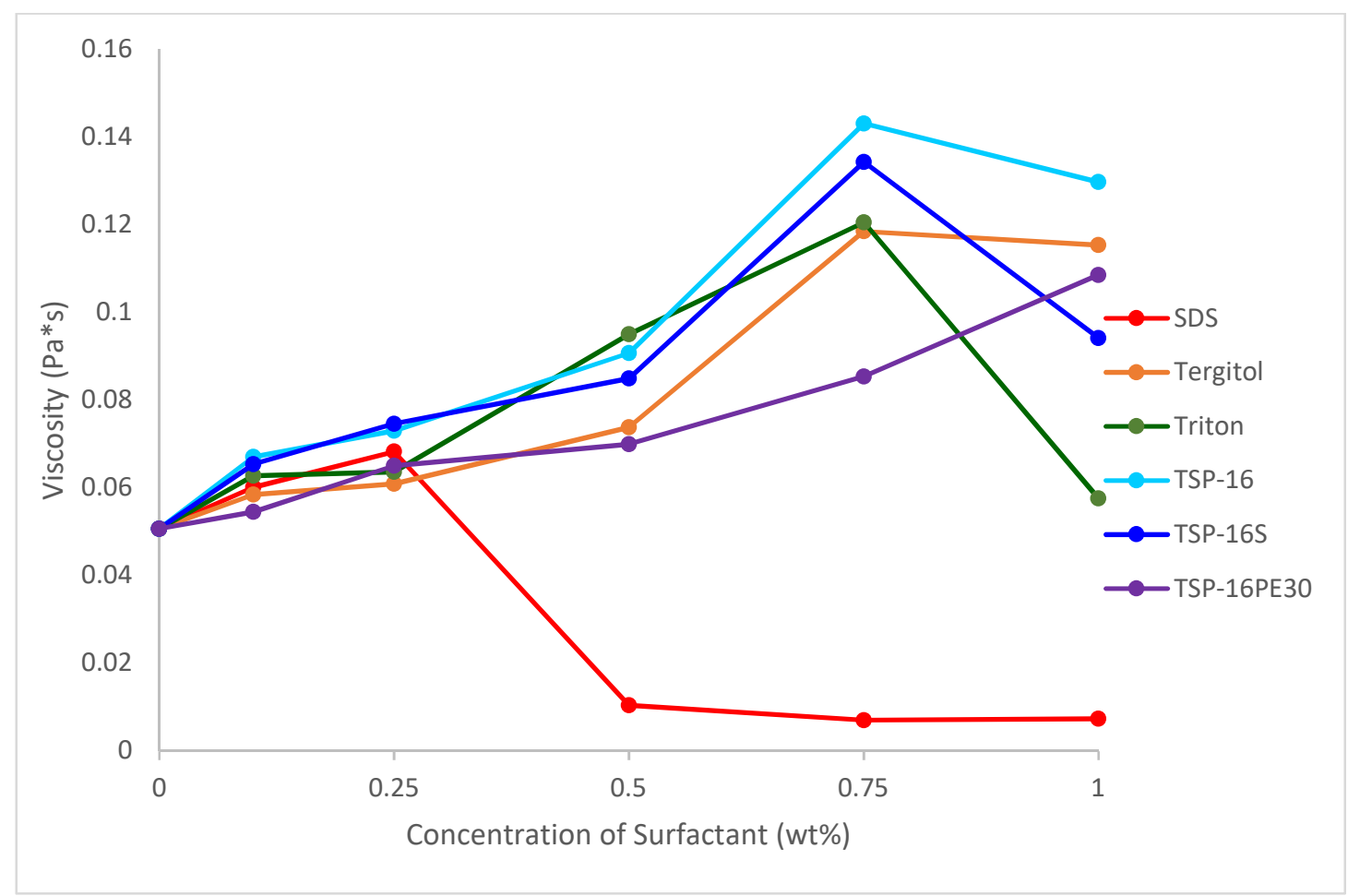

Figure 26. Viscosity values at $1000 \mathrm{~s}-1$ as a function of surfactant concentration (wt. \%) for BA/STY systems

The parabolic behavior seen in these systems at high shear rates can be attributed to the interactions between surfactant micelles and the HEUR associative network. It is important to note that although the surfactants in this system are loaded at a weight percent that is above $\mathrm{CMC}$, this is only in ideal aqueous surfactant systems. When additional associative sites like the latex particle surface or HEUR flower micelles are introduced, the required amount of surfactant required to acquire free-floating micelles is much higher. When surfactant is loaded into a system like this, surfactant molecules first associate with the highest affinity sites possible before they begin to create their own 
hydrophobic domains inside of micelles. This means there will be surfactant present freely in the solution, at the water-air interface, at the latex-water interface, and at the HEUR-water interface all prior to a noticeable number of free-floating surfactant micelles forming ${ }^{17}$.

As more surfactant micelles form, the HEUR hydrophobes begin to more readily interact with the hydrophobic domains of the micelles. At low concentrations of surfactant, micelles are loosely incorporated into the HEUR associative network, attached by the occasional free-floating HEUR hydrophobic end. As concentration of surfactant increases, eventually surfactant micelles become key points for indirect HEUR bridging sites, promoting viscosity increases to a maximum viscosity. Eventually however, at a high enough concentration of surfactant loading, excess surfactant micelles are formed, and two significant things happen. First, these excess micelles now begin to singularly occupy a significant number of HEUR hydrophobes, preventing them from forming effective bridges. Second, these micelles also repel each other to an extent. This means that freely floating micelles can now repel HEUR-associated surfactant micelles, which reduces the breadth of the associative network again. So as surfactant concentration goes from low to high, there is parabolic maximum that occurs at a nominal loading of surfactant into a HEUR system. 
3.2 Flow Sweep Analysis of BA/MMA-Surfactant-Thickener Aqueous Systems

\subsubsection{BA/MMA-HEUR Systems with $0.10 \mathrm{wt} \%$ Additional Surfactant}

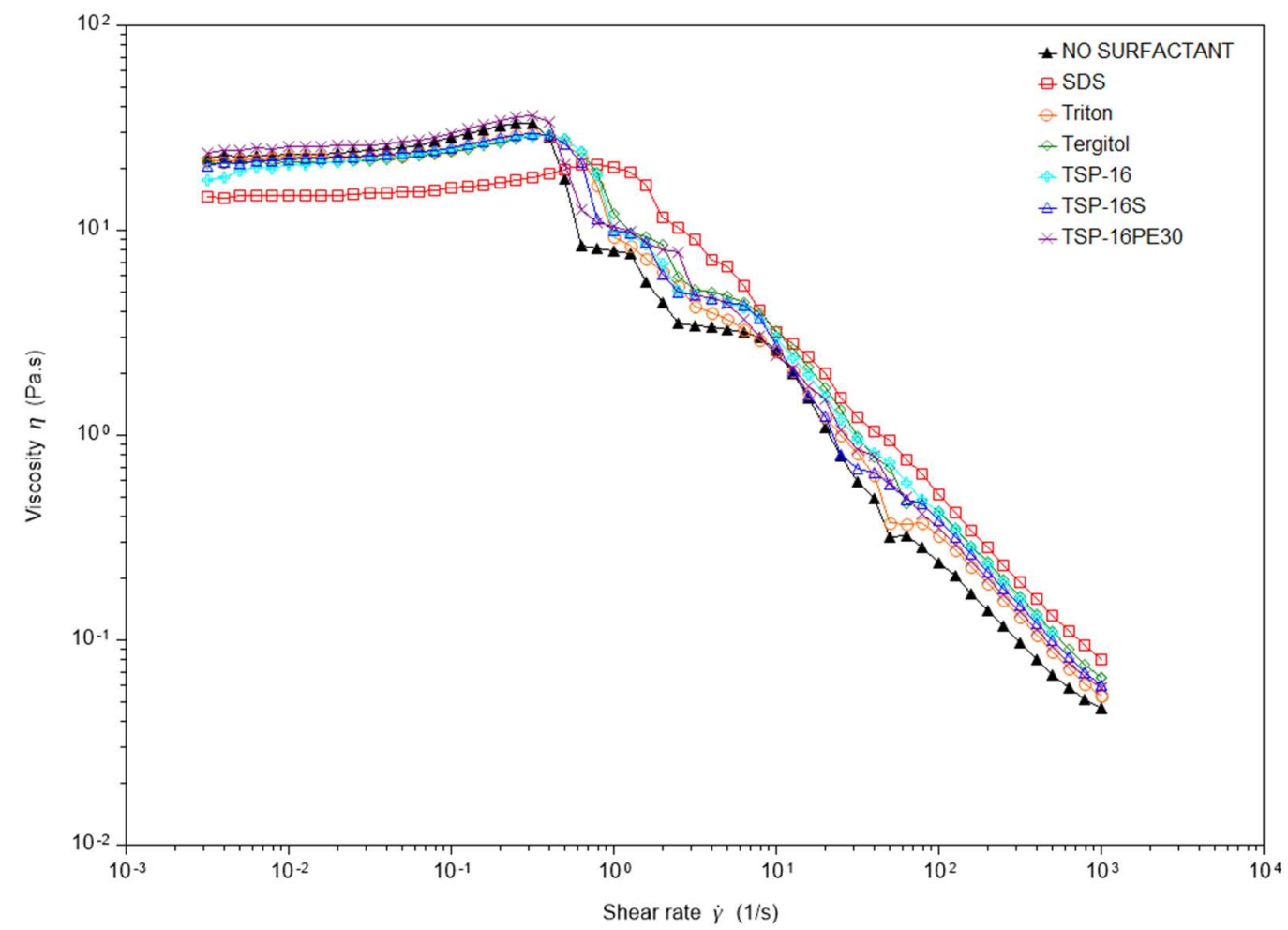

Figure 27. Flow Sweep Overlay of BA/MMA Latex at 25 vol. \%, C18-EO795 thickener at 0.5 wt. $\%$, Surfactant at $0.1 \mathrm{wt} \%$ systems.

In Figure 27, an overlay of systems with up to $0.10 \mathrm{wt}$. \% of additional surfactants can be seen. The BA/MMA samples demonstrate a very distinct behavior in Regime III with a very clearly defined multi-step profile. On the control flow sweep there are 3 distinct steps in Regime III and at the transition to Regime IV. Similar step-like behavior is seen at higher concentrations of surfactant, but the characterization of the mechanisms at play is to be determined in future work. 
Unlike the BA/STY latex system, at this weight percent of SDS, there is not an equivalent increase in viscosity over the control in Regime I. This is due to the fact that the latex is more hydrophilic and the associative thickener ends start with a lower affinity to the surface. The same mechanisms may be at play, but the overall viscosity does not cumulatively add up to be larger than the control in Regimes I and II. TSP-16PE30 however does show a slight increase over the control at low to mid shear rates. All samples show an increase in viscosity relative to the control in Regimes III and IV. Most intriguing to note is that in Regimes III and IV, the sample with SDS has the highest viscosity, most likely coming from the rightward shift of the entire profile, not necessarily some form of high-shear thickening mechanism.

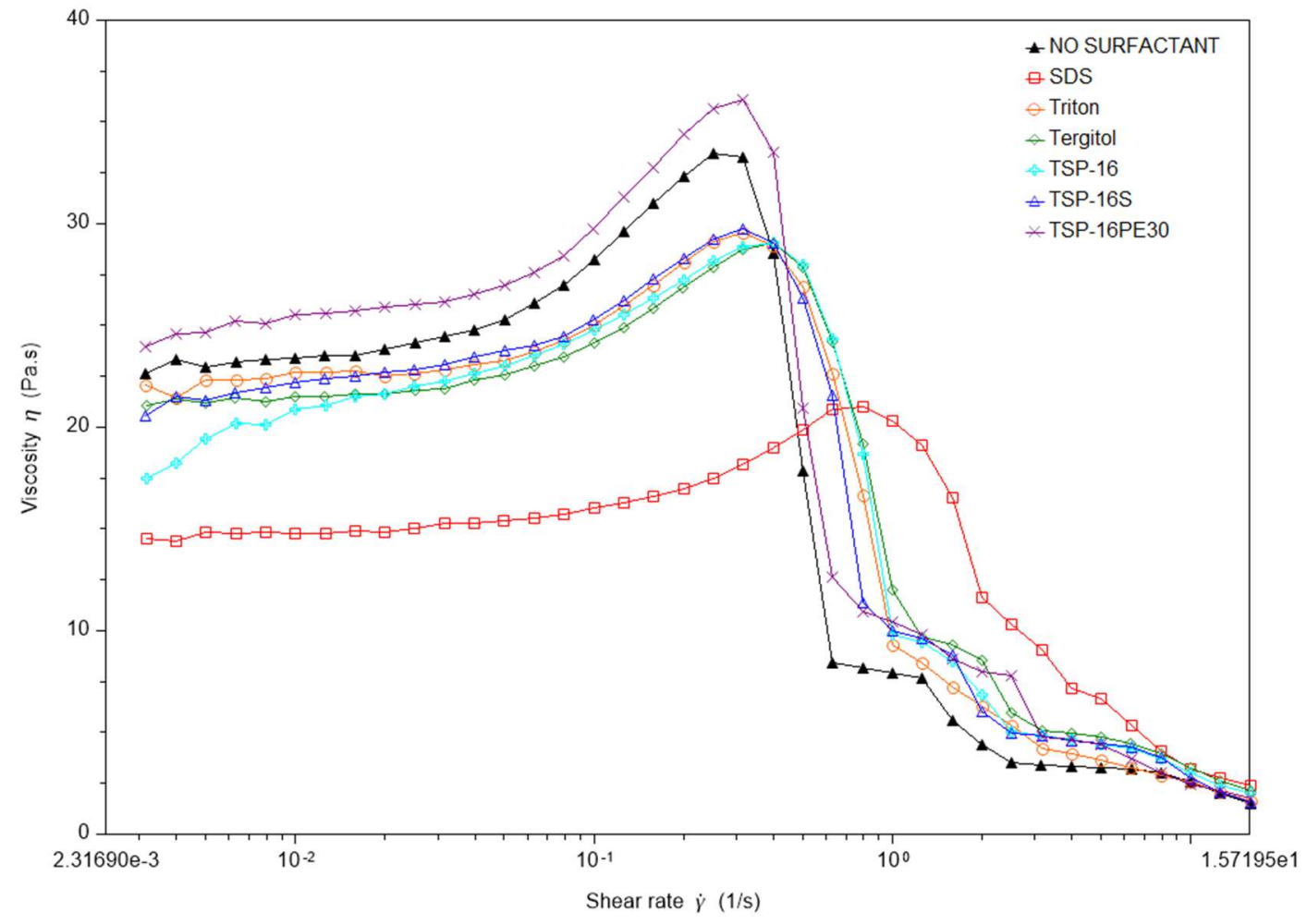

Figure 28. Regimes I and II with linear y-axis for C18-EO795/BA-MMA, with $0.10 \mathrm{wt} \%$ surfactant systems. 
Figure 28 shows Regimes I and II of the $0.10 \mathrm{wt} . \%$ systems for a good view of the ordering of the viscosities of each surfactant. SDS is lowest in viscosity and has the rightward most shifted shear-thickening maximum. TSP-16PE30 is highest in viscosity but still has a rightward shifted viscosity maximum. The remainder of the surfactant systems are so closely clustered that they are near indistinguishable at this current concentration.

\subsubsection{BA/MMA-HEUR Systems with $0.25 \mathrm{wt} \%$ Additional Surfactant}

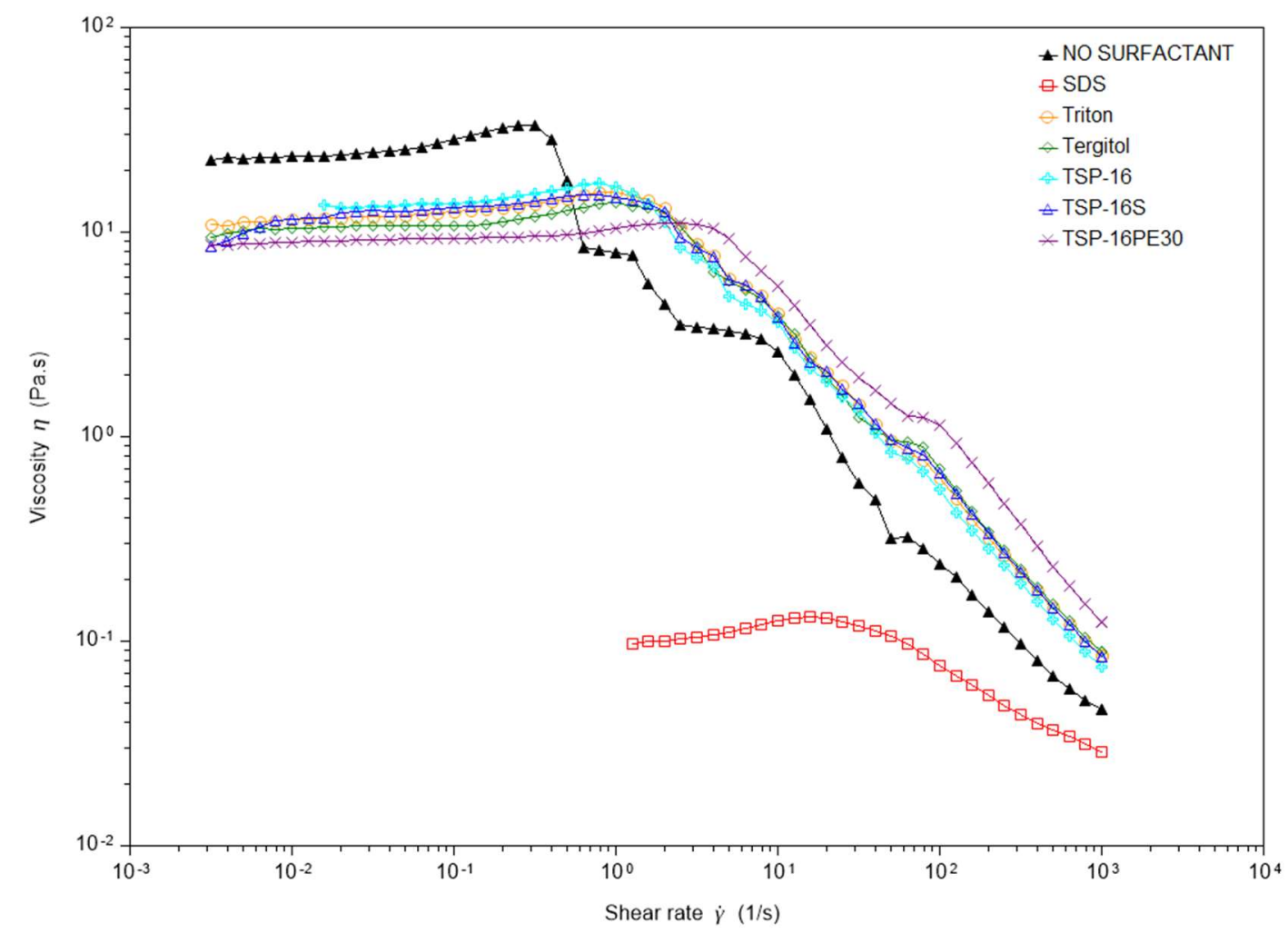

Figure 29. Flow Sweep Overlay of BA-MMA Latex at 25 vol. \%, C18-EO795 thickener at 0.5 wt. $\%$, Surfactant at 0.25 wt. $\%$ systems.

With an increase in concentration to $0.25 \mathrm{wt} \%$ of additional surfactant there is an immediate drop of the sample with SDS. The viscosity has dropped significantly below 
the control in all Regimes where data was able to be collected. However, unlike the BASTY surfactant-latex-thickener system, it is not Newtonian in behavior yet.

Another noticeable feature at this concentration is the sudden drop in the viscosity of the TSP-16PE30 sample. At $0.1 \mathrm{wt} . \%$ the sample had higher viscosity than the control in Regimes I and II. Now the sample has lower viscosity than all samples other than SDS in the same region. Beyond Regime II the sample has an increased viscosity over all samples in Regimes III and IV, again showing that rightward shift of the whole viscosity profile. Directly at the transition from Regime III to Regime IV, at $100 \mathrm{~s}^{-1}$, the step is very pronounced here for TSP-16PE30. The behavior can be seen in the other surfactant samples, but none are nearly as pronounced as the sample with TSP-16PE30. 


\subsubsection{BA/MMA-HEUR Systems with $0.50 \mathrm{wt} \%$ Additional Surfactant}

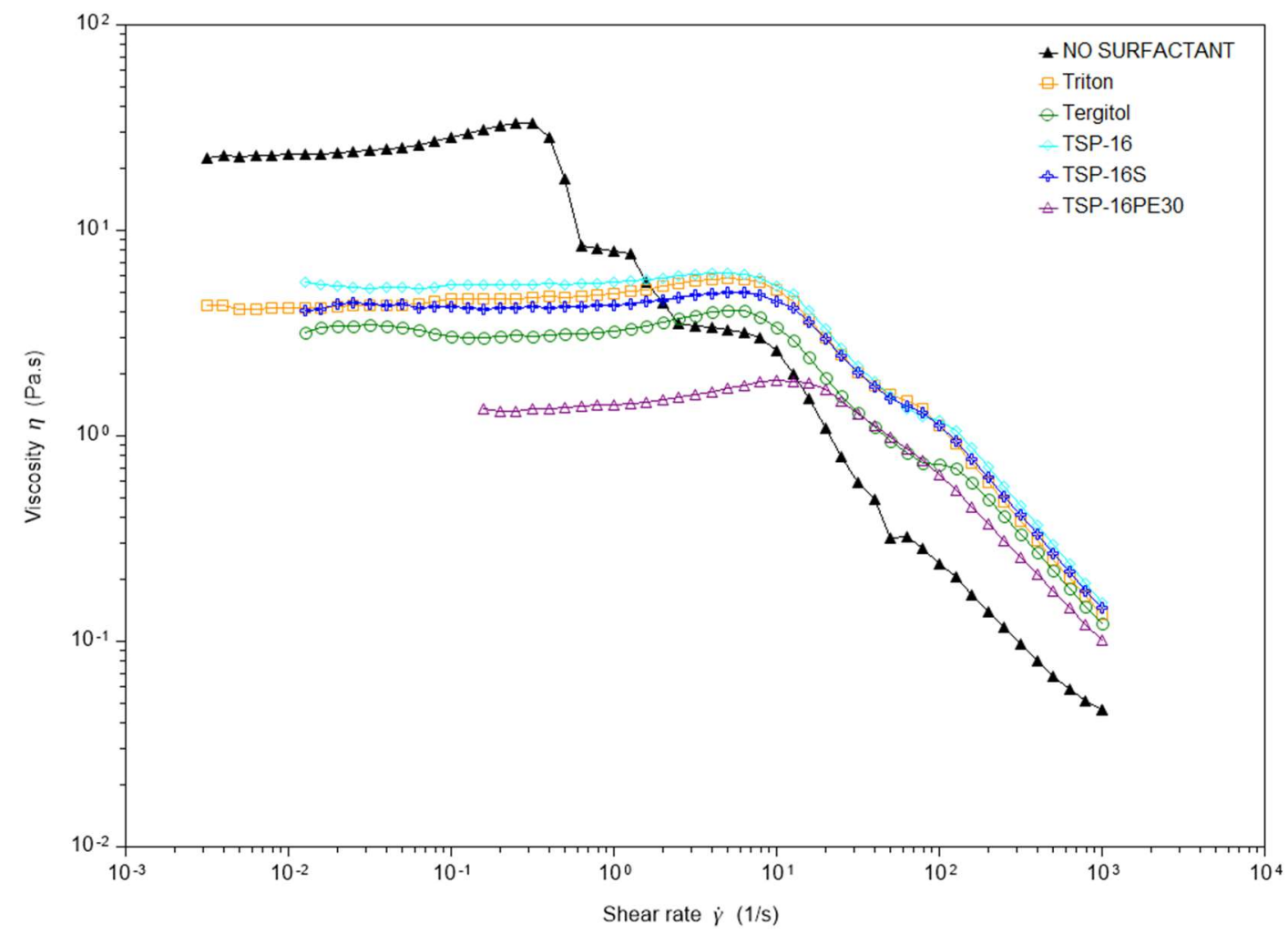

Figure 30. Flow Sweep Overlay of BA-MMA Latex at 25 vol. \%, C18-EO795 thickener at 0.5 wt. $\%$, Surfactant at 0.50 wt. $\%$ systems.

By increasing concentration of surfactant to $0.50 \mathrm{wt} \%$, a drop in viscosity can be seen in all samples. The sample with TSP-16PE30 shows a further decrease below the other four surfactants and is the least competitive of the surfactants in the BA/MMA system. The TSP-16PE30 sample also now shows a lack of the step at $100 \mathrm{~s}^{-1}$. This is much like the BA/STY system with Tergitol 15-S-40 at $1.0 \mathrm{wt}$ \%. The TSP-16PE30 sample at $0.50 \mathrm{wt} . \%$ is on its way to decreasing in viscosity to a Newtonian behavior. 


\subsubsection{BA/MMA-HEUR Systems with $0.75 \mathrm{wt} \%$ Additional Surfactant}

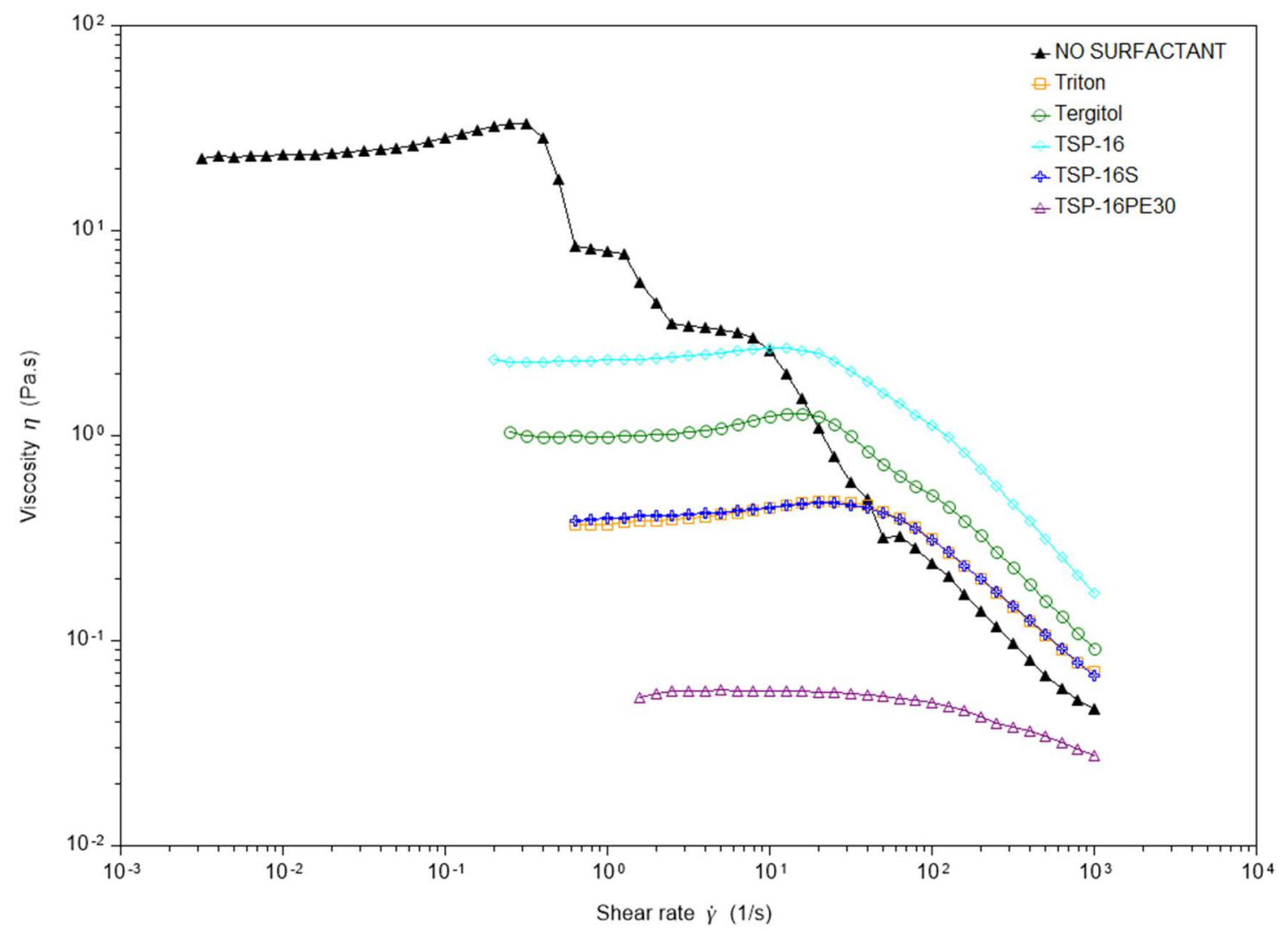

Figure 31. Flow Sweep Overlay of BA-MMA Latex at 25 vol. \%, C18-EO795 thickener at 0.5 wt. $\%$, Surfactant at 0.75 wt. $\%$ systems.

The final concentration level of samples that were measurable were at $0.75 \mathrm{wt} \%$ additional surfactant. At this level of surfactant concentration, a clear ordering of the different surfactants can be seen. The sample with Tergitol 15-S-40 is the only sample showing a prominent shear-thickening peak at this concentration. The sample with TSP16PE30 shows the greatest decrease in viscosity and almost reaches a Newtonian behavior. This near-Newtonian behavior indicates an almost complete disruption of the associative network of thickener bridges. The least disruptive surfactant for the BA/MMA latex systems is TSP-16, having the smallest overall decrease in viscosity. The 
samples containing TSP-16S and Triton X-100 are nearly matched in rheological profile. At this concentration the unique step around $100 \mathrm{~s}^{-1}$ is missing which indicates that all systems were likely close to becoming Newtonian. This can be confirmed with the analysis of the high shear viscosity values.

\subsubsection{High shear viscosity analysis of BA/MMA systems}

As can be seen in Figures 32 and 33, SDS demonstrates a peak at $0.1 \mathrm{wt} \%$ and an immediate drop at $0.25 \mathrm{wt} . \%$. The next peak is TSP-16PE30 at $0.25 \mathrm{wt} . \%$, which correlates to the disruptive behavior seen in the BA/MMA flow sweeps. Then Tergitol 15-S-40 likely has a peak that lies between 0.25 and $0.50 \mathrm{wt} . \%$. TSP-16S shows a peak at 0.75 wt. $\%$. The only peak not fully resolved in this concentration range is TSP-16 which may have a peak between 0.50 and $0.75 \mathrm{wt} . \%$ at $100 \mathrm{~s}^{-1}$ or possibly above $0.75 \mathrm{wt} . \%$ at $1000 \mathrm{~s}^{-1}$.

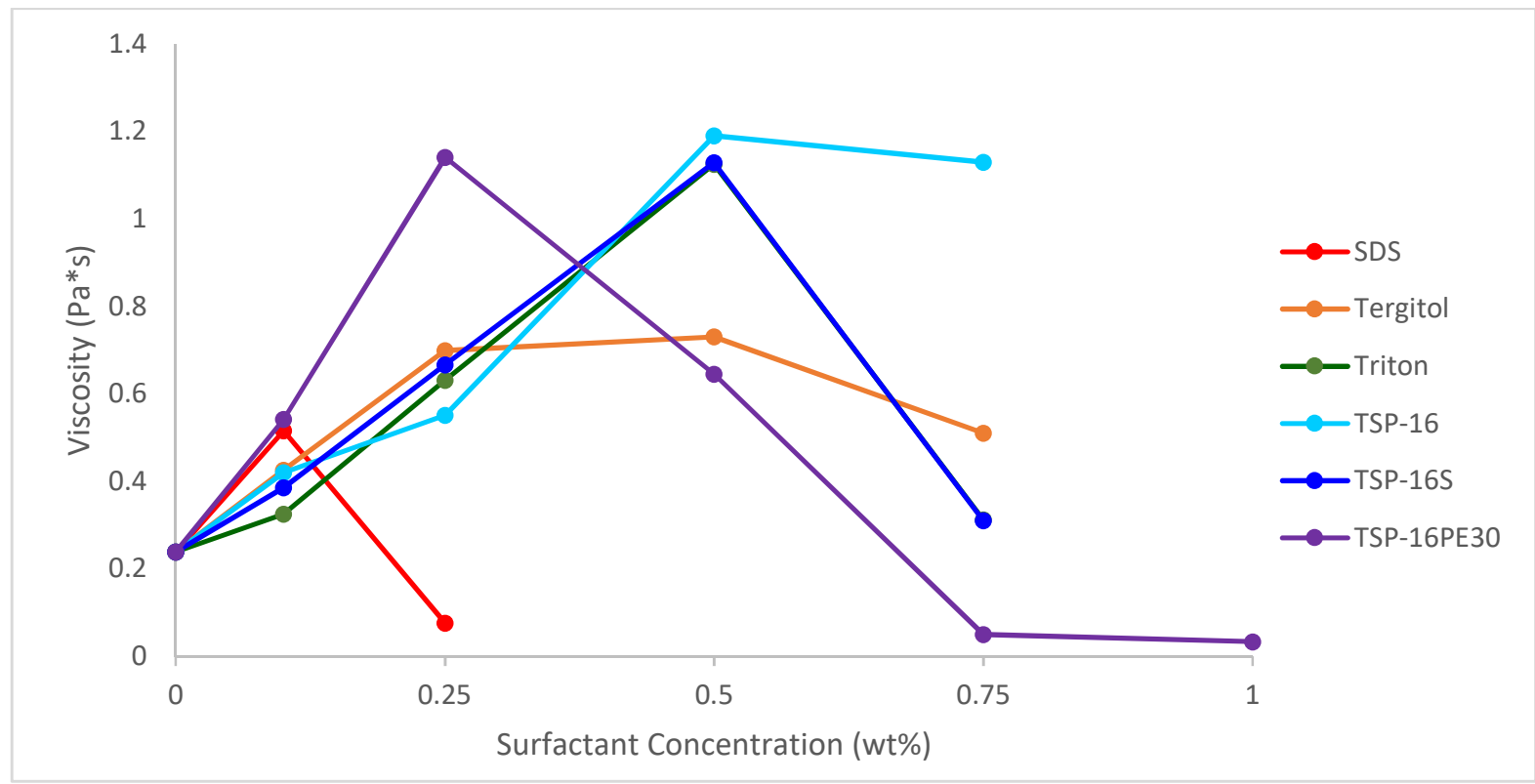

Figure 32. Viscosity values at $100 \mathrm{~s}-1$ as a function of surfactant concentration (wt. \%)

for BA/MMA systems 
Based on Figures 32 and 33 the BA/MMA systems demonstrate a relatively lower surfactant concentration where the viscosity peak is seen from the micelle-HEUR interactions. The lower surfactant concentration required to reach this viscosity maximum can be attributed to the difference in the hydrophobicity of the two latexes. BA/STY being the more hydrophobic latex, has a higher affinity for surfactant association than the BA/MMA systems. With a lower affinity for the surface of the BA/MMA, surfactant molecules more readily form favorable micelles at a lower relative concentration.

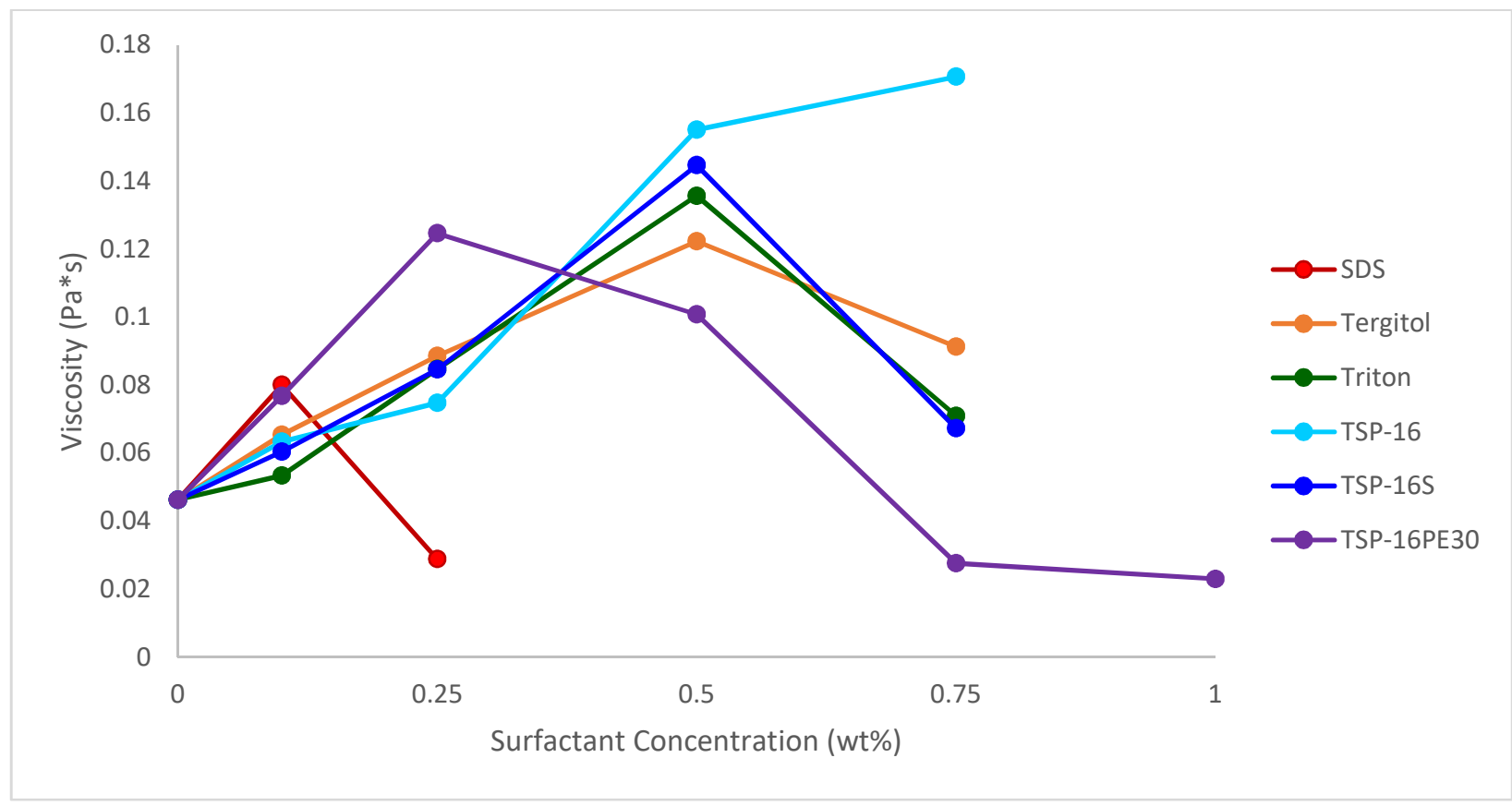

Figure 33. Viscosity values at $1000 \mathrm{~s}-1$ as a function of surfactant concentration (wt. \%) for BA/MMA systems

When the concentrations of these remaining surfactants were increased to 1.0 $\mathrm{wt} \%$, they all reached a Newtonian behavior in appearance when the glass vial was tilted. However, when viscosity measurements were performed on the rheometer, data was discontinuous. 
3.3 Comparative Flow Sweep Analysis of BA-STY and BA-MMA systems.

\subsubsection{Comparison of BA-STY-HEUR and BA-MMA Systems with $0.1 \mathrm{wt} \%$ Additional}

Surfactant

After a thorough look at each of the latex-surfactant-thickener systems

individually, it is still important to overlay the flow sweeps to see the differences between the two systems. The major trends still apply in both latex systems as will be seen in the following figures, however, there are some differences that are important to note.

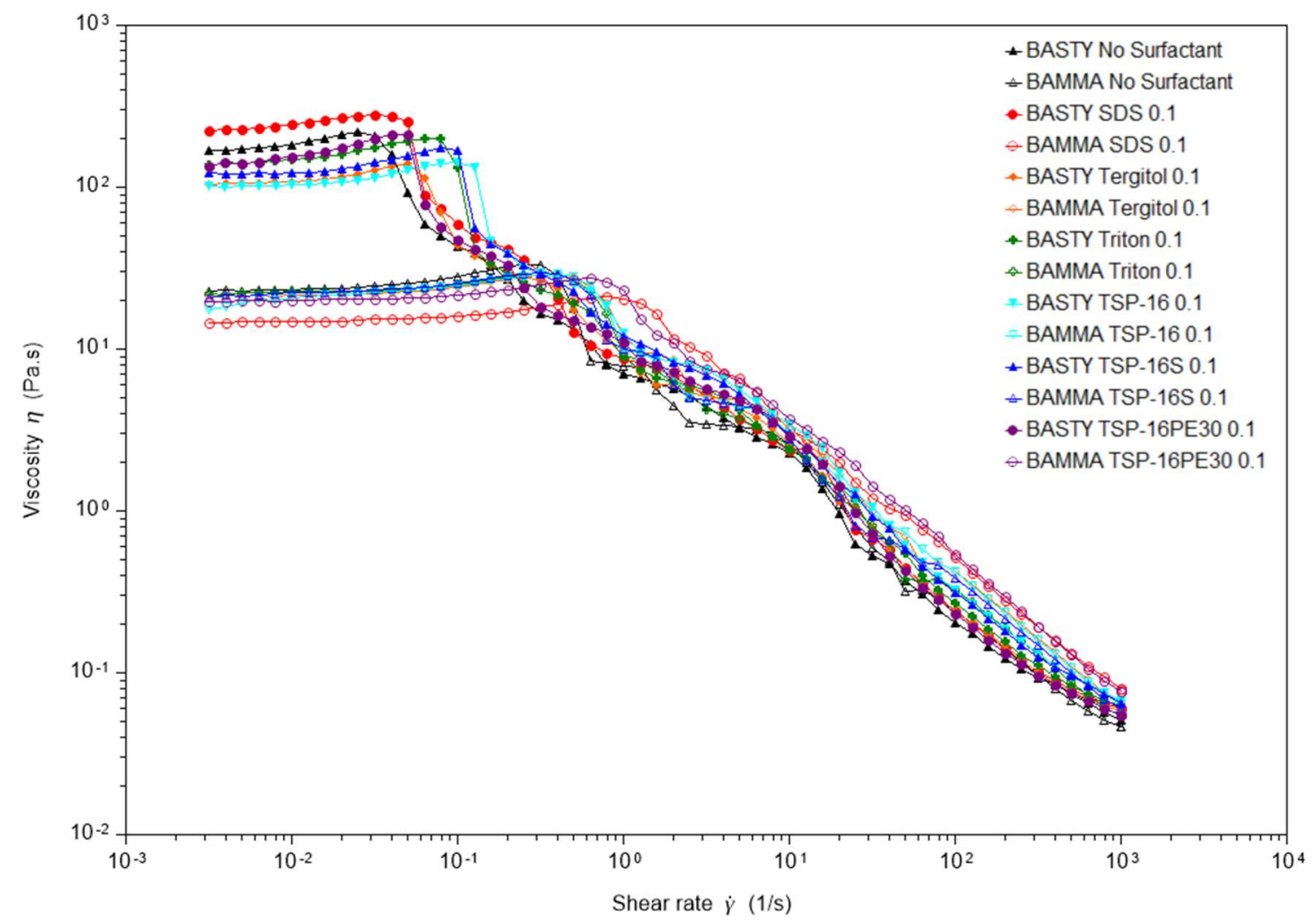

Figure 34. Flow sweep comparison of BA-STY and BA-MMA latex at 25 vol. \%, C18EO795 thickener at $0.5 \mathrm{wt} \%$, surfactant at $0.1 \mathrm{wt}$. \% systems. 
At $0.10 \mathrm{wt} \%$ of additional surfactant, the difference between the overall viscosities can immediately be seen. In Regimes I and II there is a difference of an entire logarithmic decade in viscosity. Additionally, the shear-thickening maximum is shifted more than a whole logarithmic decade to the right to a higher shear rate. Higher shear rates represent a smaller time scale which is to be expected for a more hydrophyllic latex and why the BA-MMA samples have a lower overall viscosity than the BA-STY samples at the same concentration of $0.5 \mathrm{wt} \%$ of HEUR thickener. With the shift into Regimes III and IV there is a reclustering of all systems to a highly linear shear thinning region.

In the BA-STY systems, the sample with SDS shows an increase in viscosity in Regime I over the control, something that is not seen in the BA-MMA latex system. This is likely due to the already lower affinity of the BA-MMA to the hydrophobic associative ends of both surfactant and thickener molecules. It is likely that at a much lower concentration of SDS surfactant, there may be a visible increase in viscosity over that of the control in Regimes I and II, but these values were not explored in this work.

The reason for these differences in behavior are due to the differences in hydrophobicity. BA-MMA, the most hydrophyllic of the two latexes, is more loosely associating with the hydrophobic ends of the thickener chains, which is lowering the overall number of active bridges. This is what leads to the decrease in viscosity at low shear rates. Additionally, with fewer active bridges and associated ends on the surface of a latex particle, the more free surface area there is for surfactant to bind. It is also then likely that the surfactants have a higher affinity and are more competitive than the hydrophobic ends of the thickener. As concentration of surfactant increases, this trend stays true where low shear rate viscosity decreases. 
Surfactant

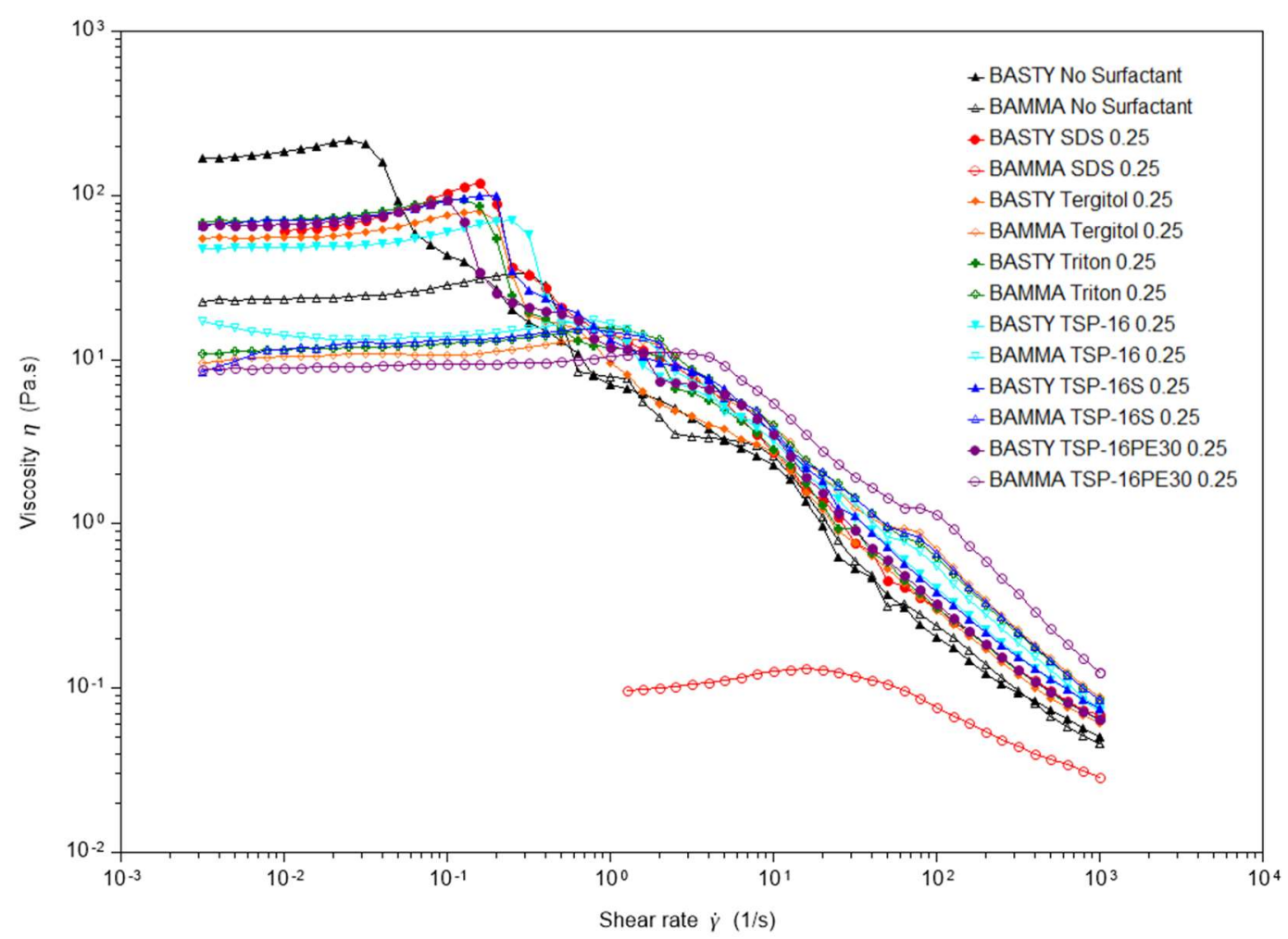

Figure 35. Flow sweep comparison of BA-STY and BA-MMA latex at 25 vol. \%, C18EO795 thickener at $0.5 \mathrm{wt} \%$, surfactant at $0.25 \mathrm{wt} . \%$ systems.

When comparing the two systems at the increased concentration of $0.25 \mathrm{wt} . \%$, the next difference in the two systems can be seen. With the BA-MMA system, the sample with SDS has already dropped significantly in viscosity, whereas the sample with SDS and BA-STY latex has not yet shown this dramatic decrease. The BA-MMA sample with SDS does not show a full transition to the full Newtonian behavior at this concentration, but an increase to $0.50 \mathrm{wt} . \%$ did not have high enough torque values on the rheometer to provide meaningful results and as such that sample was not included in the next figure. 
The shift from 0.1 wt. $\%$ to 0.25 wt. \% very clearly shows the effects of the difference in hydrophobicity of the two latexes. The more hydrophobic the surface of the latex particle, the higher affinity that the associative ends of the thickener will have when forming bridges. As the affinity between the two ingredients increases, the longer that transient bridging networks will last and thus the higher the viscosity of the system. As affinity is higher between the two species, the system can withstand stronger shear forces before the equilibrium rate of thickener associations is exceeded.

3.3.4 Comparison of BA-STY-HEUR and BA-MMA Systems with 0.50 wt\% Additional Surfactant

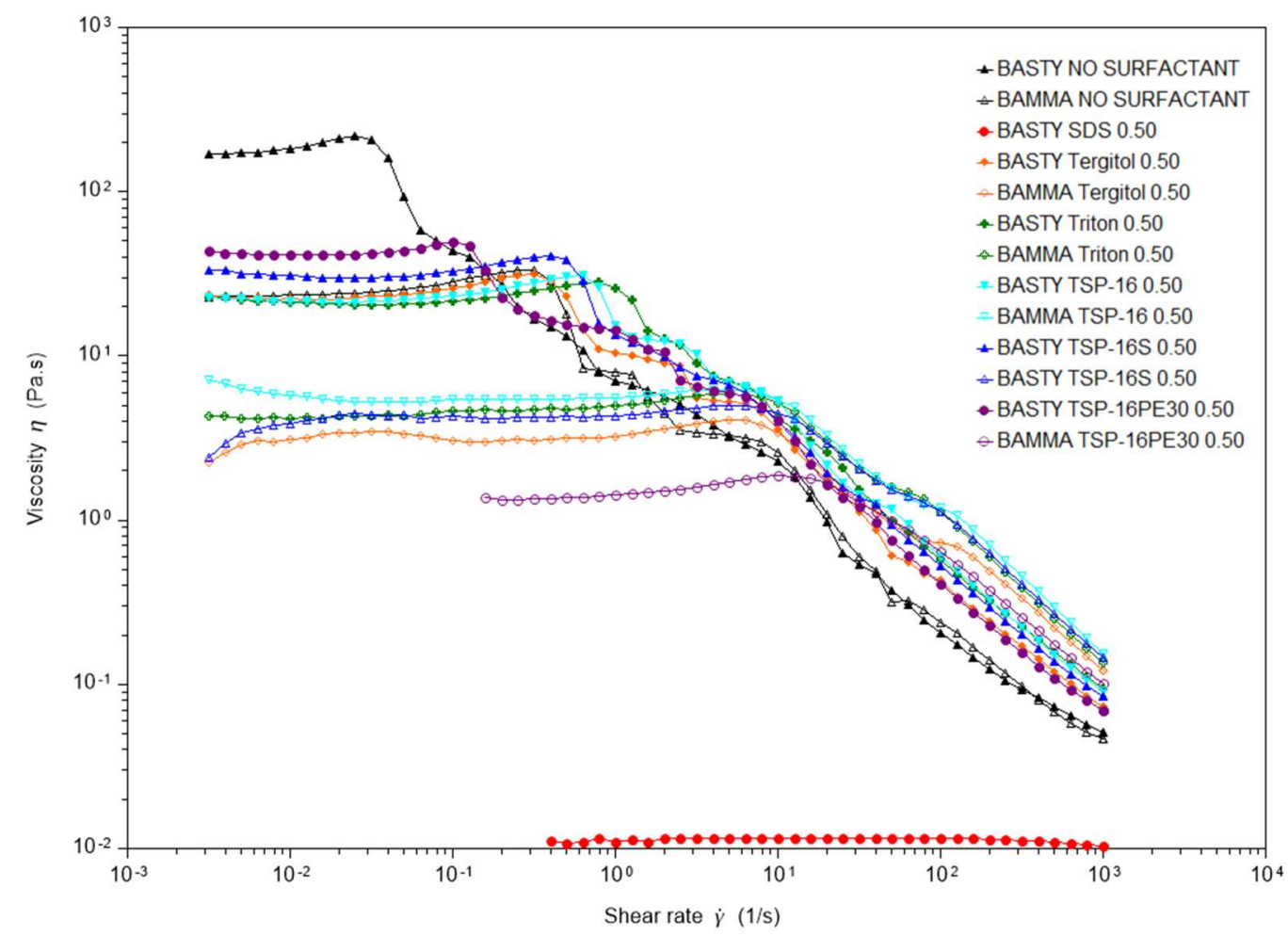

Figure 36. Flow sweep comparison of BA-STY and BA-MMA latex at 25 vol. \%, C18EO795 thickener at $0.5 \mathrm{wt} \%$, surfactant at $0.50 \mathrm{wt} . \%$ systems. 
As surfactant concentration further increases, some more distinct differences start to arise. The first one to take note of is the shift of the sample with SDS to a Newtonian behavior in the BA-STY system. Being the more hydrophobic latex, the SDS surfactant does not outcompete the associative ends of the thickener molecules as readily as it does with the hydrophyllic latex system.

The next difference to note is the samples with TSP-16PE30. In the system with BA-STY, the sample has a higher viscosity in Regimes I and II than the other surfactants, but in the BA-MMA system, the TSP-16PE30 sample is below the remaining surfactant samples in the same region. This is the most noticeable difference in regards to ordering of samples, but it is not the only sample that changes order with a switch in latex. 

Surfactant

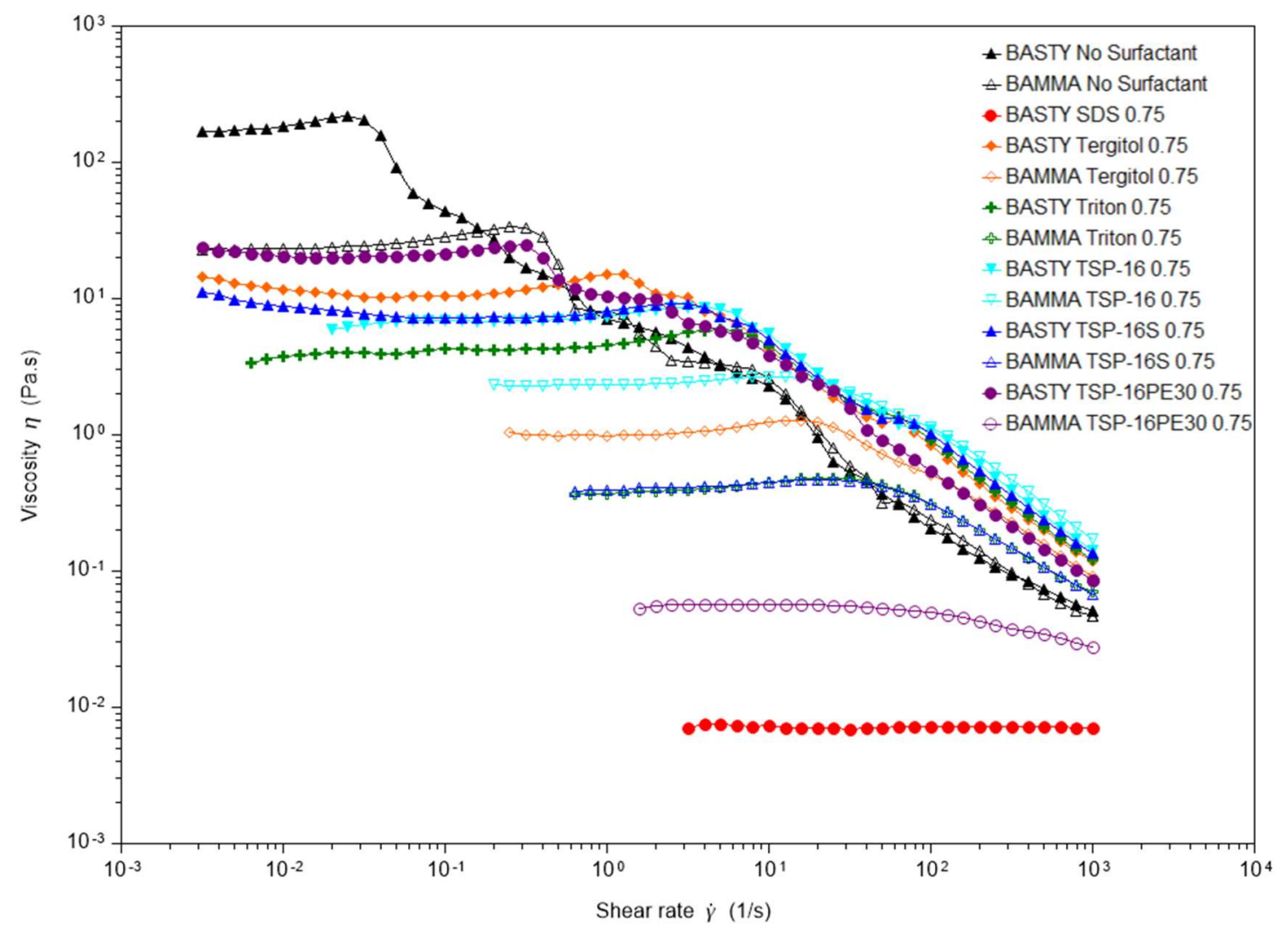

Figure 37. Flow sweep comparison of BA-STY and BA-MMA latex at 25 vol. \%, C18EO795 thickener at $0.5 \mathrm{wt} \%$, surfactant at $0.75 \mathrm{wt} . \%$ systems.

The final concentration that can be compared across the two latex systems is at $0.75 \mathrm{wt} \%$ of additional surfactant. At this concentration of surfactant, the difference in the latexes can be very clearly seen. All samples containing the BA-MMA latex particles have significantly lower viscosities than their equivalent BA-STY counterparts.

Additionally, ordering of affinities of the different surfactants can be seen. For the BA-MMA systems, the order from highest affinity to latex surface to lowest affinity is as such: SDS, TSP-16PE30, TSP-16S/Triton X-100, Tergitol 15-S-40, and finally TSP-16. 
For the BA-STY systems, the order from high to low is as such: SDS, Triton X-100, TSP-16, TSP-16S, Tergitol 15-S-40, and TSP-16PE30. In the BA-MMA systems, it appears as though there may be a correlation between ionic nature of the surfactant and the ability to interfere with the thickening mechanism. Based on the ordering of the samples based on decreases in viscosity, ionic surfactants interfere with the thickening mechanism more readily than non-ionic surfactants. However, in the BA-STY systems, this trend does not hold true.

\subsection{Dynamic Data Analysis of BA/STY-HEUR-Surfactant systems.}

Dynamic analysis of these systems further sheds light on nature of how the LatexHEUR-Surfactant networks are working. The two types of dynamic analyses performed with the rheometer include amplitude sweeps and frequency sweeps. With the amplitude sweeps, an analysis of the moduli behavior can be performed. For the scope of this work only the storage modulus will be analyzed for each system. The second type of dynamic rheological analysis performed was frequency sweep analysis. By plotting the phase angle as a function of frequency, the solid-like and liquid-like behavior of these systems can be quantified.

\subsubsection{Dynamic Data for BA/STY-HEUR-Surfactant System with $0.10 \mathrm{wt} \%$ Additional} Surfactant

At 0.1 wt. $\%$ of surfactant loading into the BA/STY systems, there is a clear set of peaks in all systems with additional surfactant. At low strain values all 6 surfactant systems have a distinct storage modulus peak as can be seen in Figure 38. At low strains all systems are high elastic which is to be expected as low strains would not be sufficient to disrupt or break apart the associative network. It is important to note that although the 
current plot does not display it, the peak of the control with no surfactant is likely only beyond the lower limit of this experiment set and will be fully resolved in future work. At higher strains above $50 \%$ all systems level out to a minimal storage modulus meaning that a viscous behavior dominates. This can be validated as there were shear thinning behaviors in the flow sweeps for all of these systems at high shear rates.

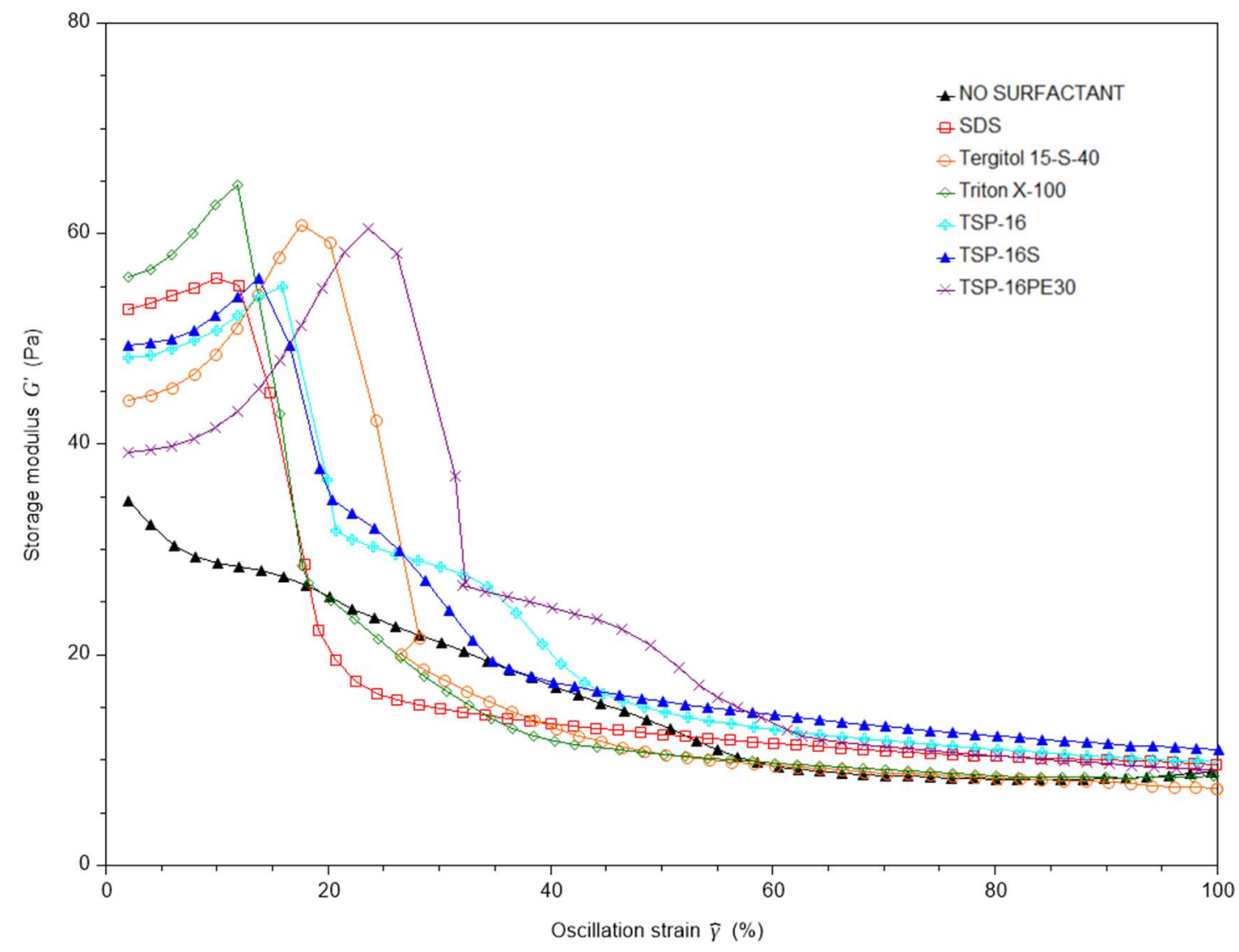

Figure 38. Linear amplitude sweep storage moduli overlay of BASTY-HEUR-Surfactant system at $0.1 \mathrm{wt} \%$ of additional surfactant.

The phase angle plot in Figure 39 reveals that all of these systems respond at all frequencies much like the control. All systems start with highly viscous behavior and then there is a distinct minimum seen in all samples. At this minimum in phase angle, it 
indicates a region of elastic, solid-like behavior. This would correspond to the rate of stretching and coiling of the HEUR associative network leading up to the shearthickening peak seen in the flow sweep plots for these systems. As the associative network begins to break apart there is a return to more fluid-like behavior, which was also seen with the shear-thinning regions in the flow sweep analysis.

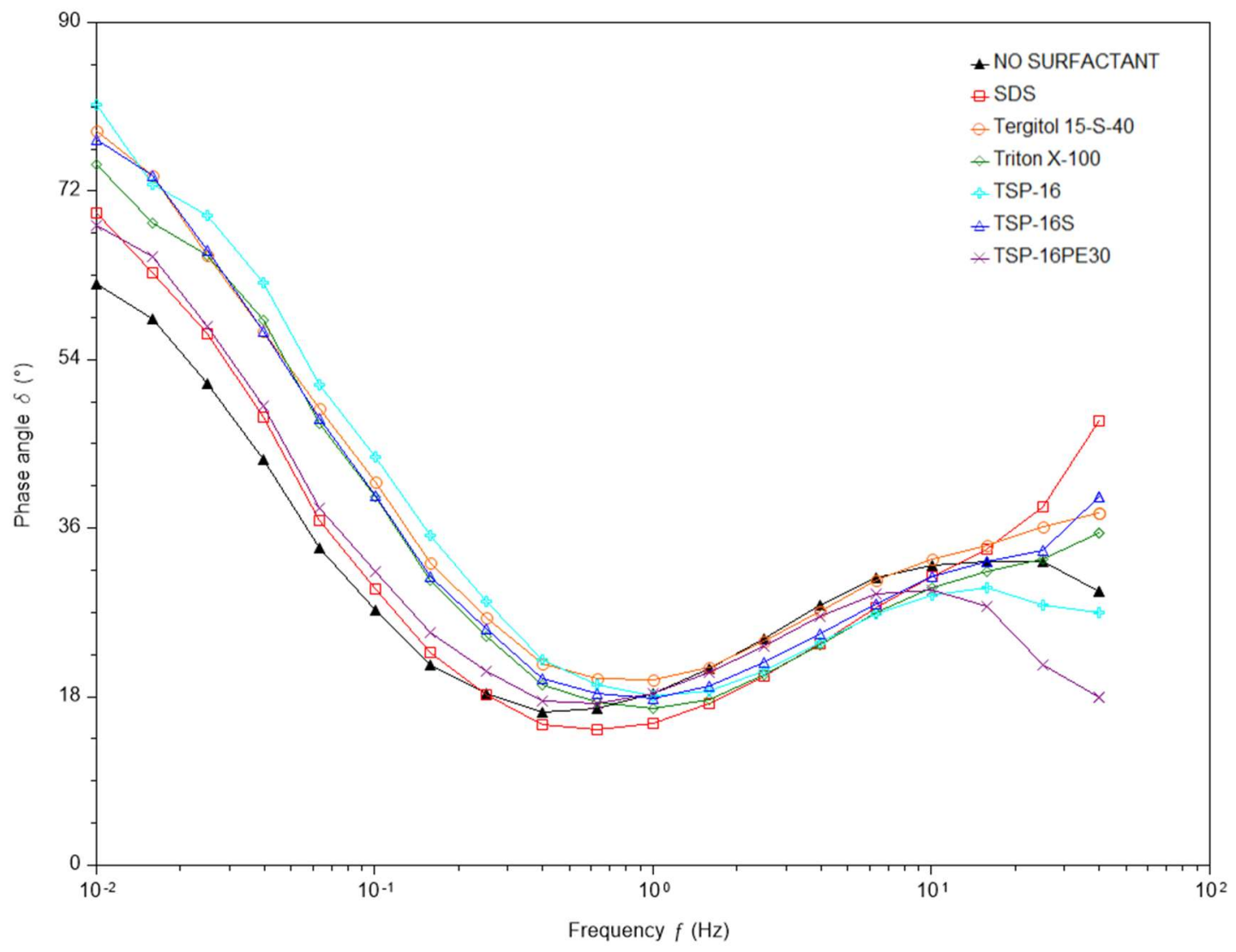

Figure 39. Phase angle plot for BASTY-HEUR-Surfactant system at $0.1 \mathrm{wt} \%$ of additional surfactant. 
3.4.2 Dynamic Data for BA/STY-HEUR-Surfactant System with $0.25 \mathrm{wt} \%$ Additional Surfactant

With an increase in surfactant loading from $0.1 \mathrm{wt}$ \% to $0.25 \mathrm{wt} . \%$ there is a clear shift of several peaks to higher strain values. In Figure 40, the two systems that respond most dramatically to an increase in surfactant loading are the samples with additional SDS and TSP-16 nonionic surfactant. The reason behind the shifts are likely different, but in both cases, these shifts to higher strain values correspond with the shift of the shear-thickening maximums seen in the flow sweep plots. The remainder of the surfactant systems do not see this same dramatic shifting to higher strains, but they do see an upward shift in the storage modulus to higher values.

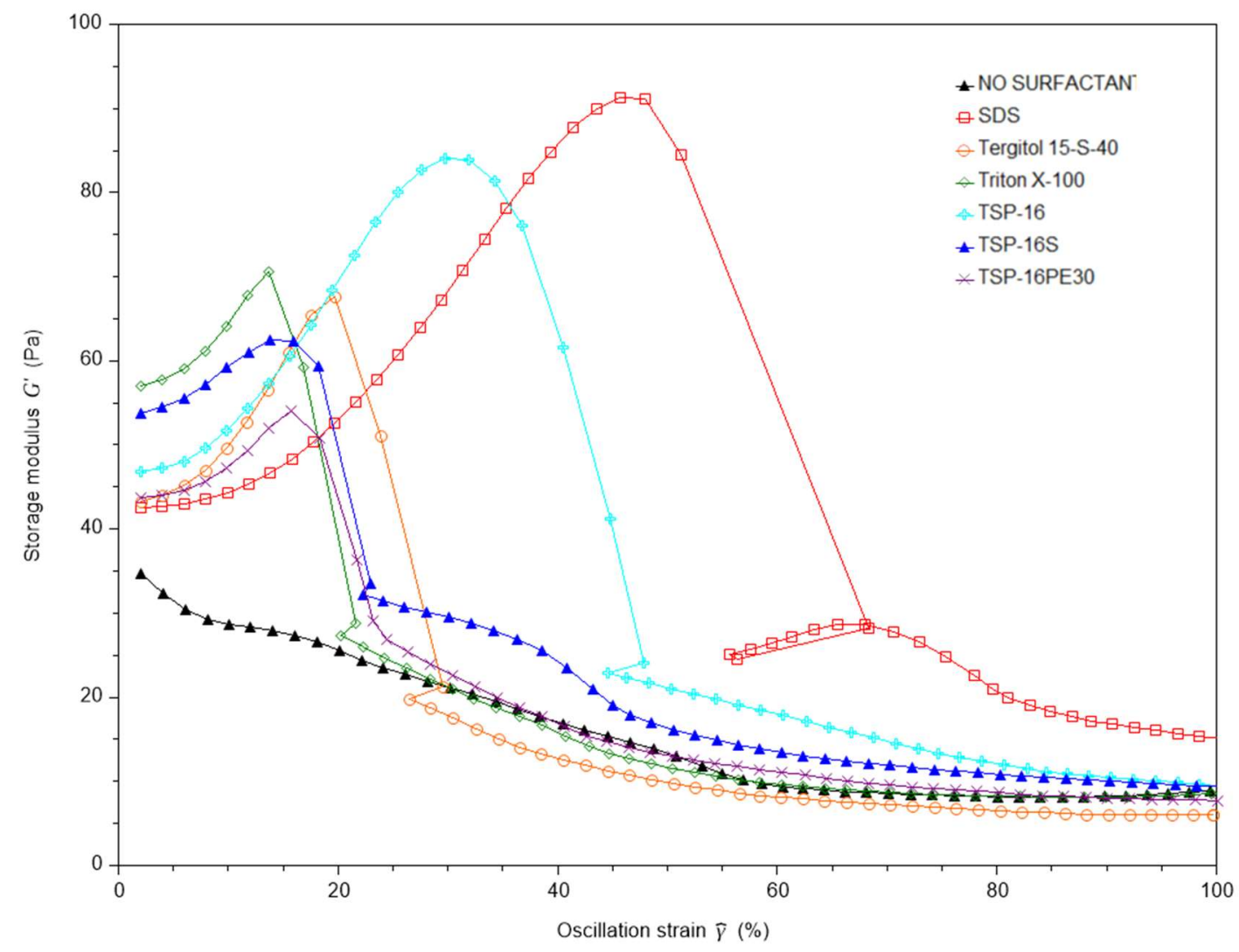

Figure 40. Linear amplitude sweep storage moduli overlay of BASTY-HEUR-Surfactant system at $0.25 \mathrm{wt} \%$ of additional surfactant. 
Figure 41 shows the phase angle behaviors for the systems at $0.25 \mathrm{wt} . \%$ surfactant loading with the BA/STY latex. At this concentration of additional surfactant there is a shift of all 6 systems away from the control. All 6 systems remain viscous in nature with a high phase angle for a slightly wider range of frequencies than the systems at 0.1 wt. \%. All systems still show an elastic minimum, but the minimum has shifted to a higher average frequency.

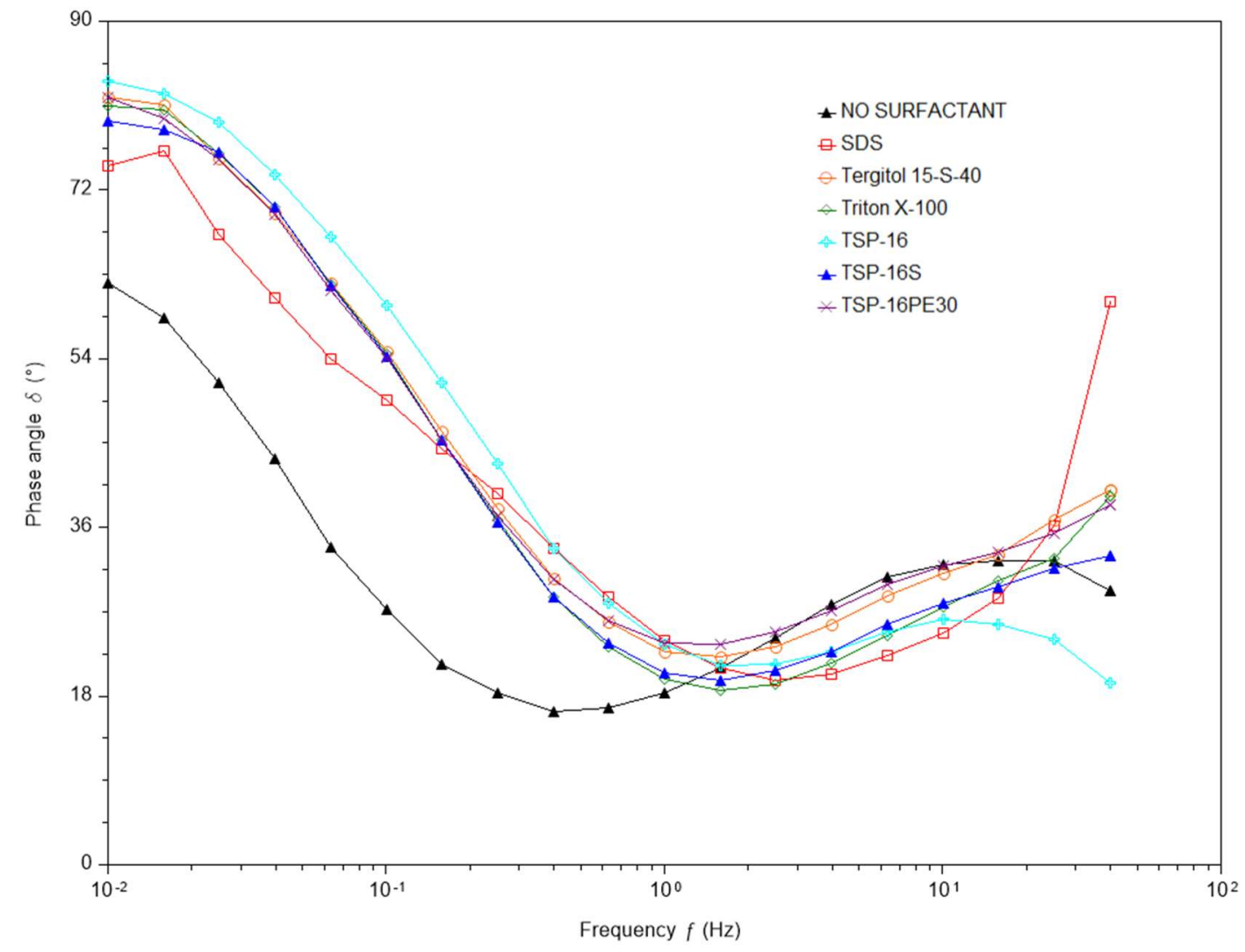

Figure 41. Phase angle plot for BASTY-HEUR-Surfactant system at $0.25 \mathrm{wt} \%$ of additional surfactant. 


\subsubsection{Dynamic Data for BA/STY-HEUR-Surfactant System with $0.50 \mathrm{wt} \%$ Additional}

Surfactant

At $0.50 \mathrm{wt} . \%$ of surfactant loading a peak increase and shift is seen from all systems except for TSP-16PE30. This correlates with the data seen in the flow sweep plots as TSP-16PE30 showed the least disruptive behavior for the BA/STY-HEUR systems. At these modulus peaks seen in Figure 42, the systems are behaving elastically. After the peak, the system returns to a low modulus indicative of the transition back to viscous behavior at similar values as the control at high strain $\%$. The next thing of note at this concentration is the shift of the sample with SDS to a completely featureless viscous response with a storage modulus of zero at all strain \% values. This correlates with the system becoming Newtonian in behavior in the flow sweep plot. The final thing that stands out in this and following amplitude sweep plots is what appears to be a doubling back of the modulus. This behavior in the plot is a result of a limitation of the rheometer. When the rheometer determines the next strain value to apply to the system, it extrapolates information from the previous trends in the data. With such steep drops in modulus at this high level of resolution of curve, the system overshoots the desired target value. This however is only a minor limitation as the software automatically adjusts and reacquires a data point at the correct desired strain $\%$ value. 


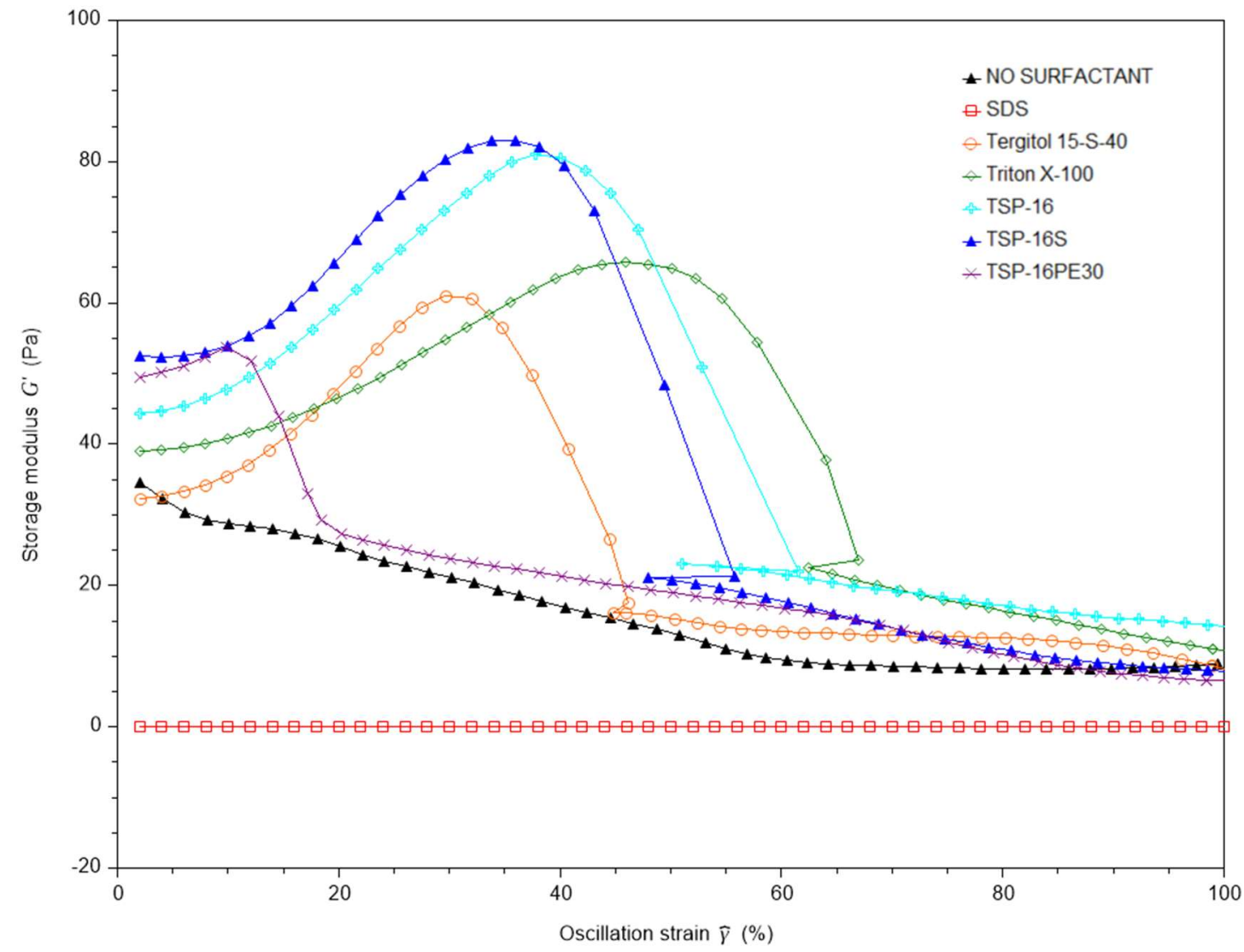

Figure 42. Linear amplitude sweep storage moduli overlay of BASTY-HEUR-Surfactant system at $0.50 \mathrm{wt} \%$ of additional surfactant.

The linear amplitude sweep correlates well with the frequency sweep for these same systems at $0.50 \mathrm{wt} . \%$ loading of additional surfactant. In the phase angle plot in Figure 43 , there is a continued shift of the elastic minimum to a higher frequency, which lines up with both the strain sweep and the flow sweep data for this system. The data for TSP-16 is odd but replicable and can be studied further in future work to determine the cause of this. 


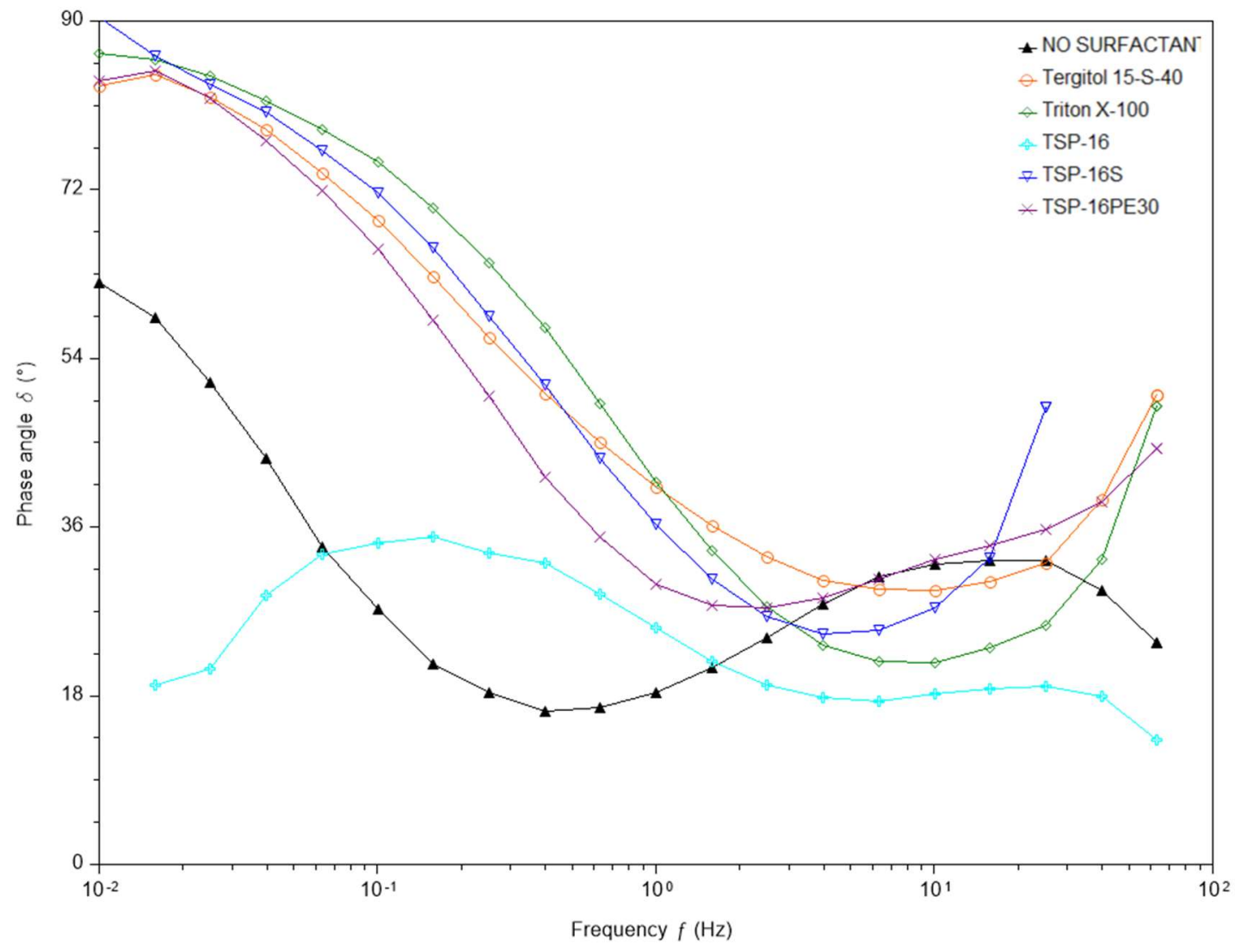

Figure 43. Phase angle plot for BASTY-HEUR-Surfactant system at $0.50 \mathrm{wt} \%$ of additional surfactant.

3.4.4 Dynamic Data for BA/STY-HEUR-Surfactant System with $0.75 \mathrm{wt} \%$ Additional Surfactant

As concentration of surfactant is increased to $0.75 \mathrm{wt} . \%$ in the BA/STY systems, a majority of samples now have become significantly less elastic. The samples with Triton X-100, TSP-16, and TSP-16S now show a very low modulus value at most strain values. It is likely that the peak lies somewhere at strain \% values above $100 \%$ and were outside of the scope of this work. Further work will be performed to fully resolve these extended peaks. The shift to lower modulus values correspond to the major decreases in viscosity seen in the flow plots from the semi-steady rheological analysis. A response 
from the TSP-16PE30 sample is seen with an increase in modulus as well as a beginning shift to higher strain $\%$. The sample with Tergitol $15-\mathrm{S}-40$ now has a broad peak roughly near $60 \%$ strain and seems to be responding just like the other samples. Also SDS can be seen with a storage modulus value of zero again at this concentration.

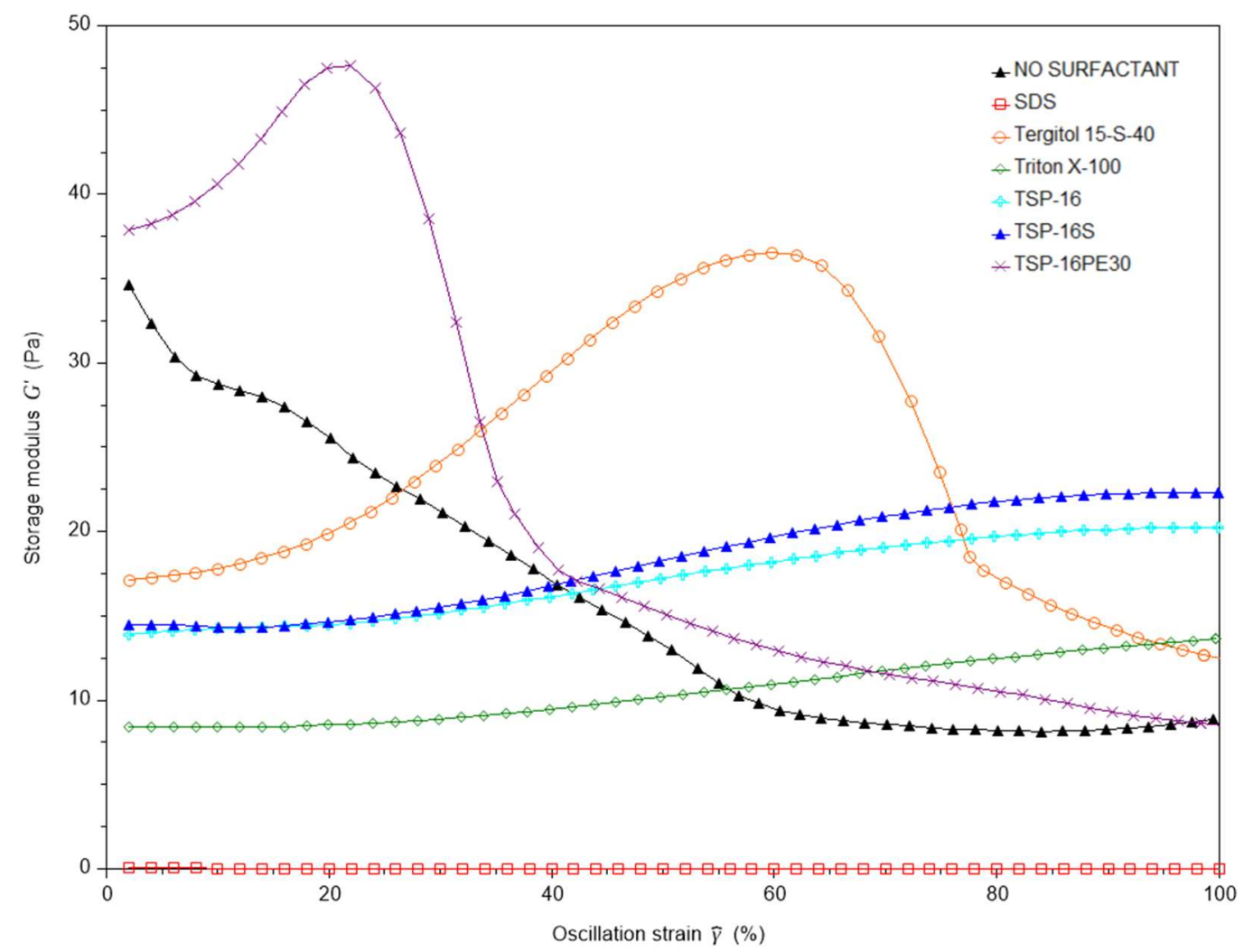

Figure 44. Linear amplitude sweep storage moduli overlay of BASTY-HEUR-Surfactant system at $0.75 \mathrm{wt} \%$ of additional surfactant.

In Figure 45, the phase angle plot for this level of surfactant loading can be seen. At 0.75 wt. \% surfactant loading, all systems are now responding highly viscously up to the elastic minimum at approximately $100 \mathrm{~Hz}$. Between 0.01 and $10 \mathrm{~Hz}$ the systems are significantly more viscous than the system with only latex and associative thickener. 


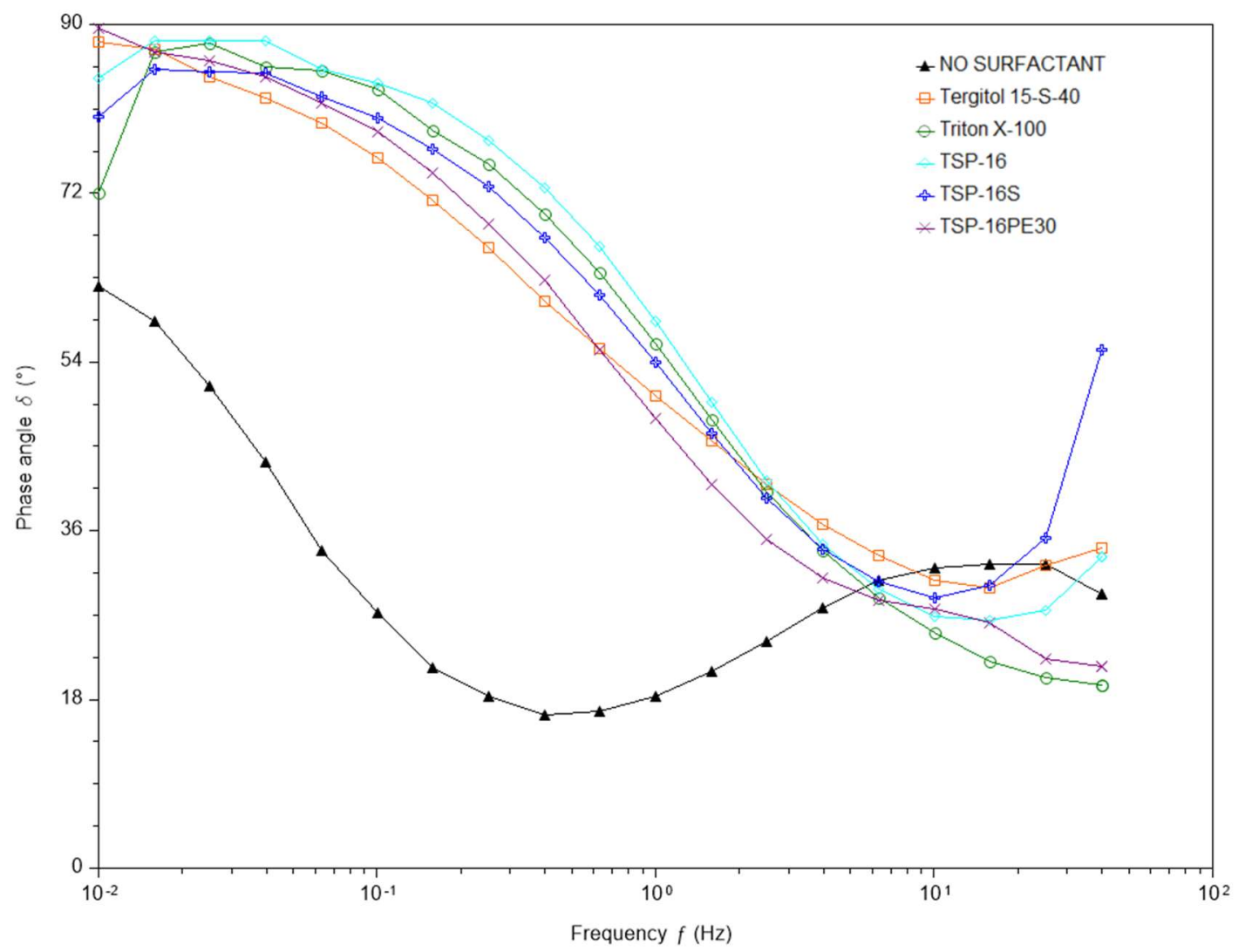

Figure 45. Phase angle plot for BASTY-HEUR-Surfactant system at $0.75 \mathrm{wt} \%$ of additional surfactant.

3.4.5 Dynamic Data for BA/STY-HEUR-Surfactant System with $1.0 \mathrm{wt} \%$ Additional Surfactant

At 1.0 wt. \% loading, 5 out of the 6 systems now show a near complete lack of storage modulus. TSP-16PE30 is still showing a shift to higher strain \% values. This strain sweep corresponds to the $1.0 \mathrm{wt}$ \% flow sweep for BA/STY. SDS has the lowest modulus and TSP-16PE30 has the highest modulus which lines up with SDS being Newtonian and TSP-16PE30 still demonstrating a complete shear-thickening peak. 


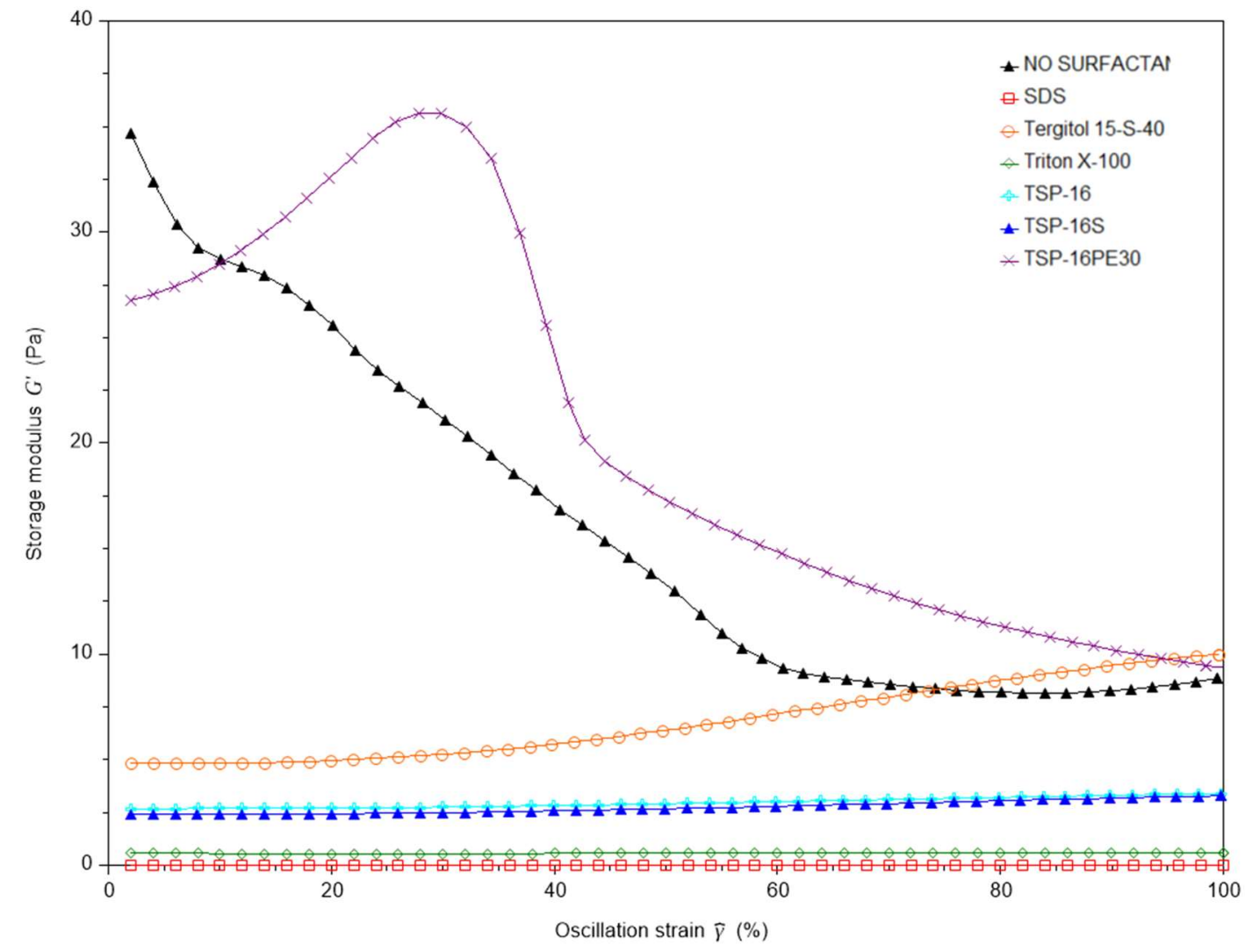

Figure 46. Linear amplitude sweep storage moduli overlay of BASTY-HEUR-Surfactant system at $1.0 \mathrm{wt} \%$ of additional surfactant.

Figure 47 shows the final phase angle plot for BASTY systems and all surfactants at this concentration show highly viscous behavior with only the sample with TSP$16 \mathrm{PE} 30$ shows a crossover with the control at high frequencies. All other samples however demonstrate a more viscous response than the control with no crossover on this plot. This correlates with the decreases of viscosity as surfactant concentration increases in the flow sweep analysis. 


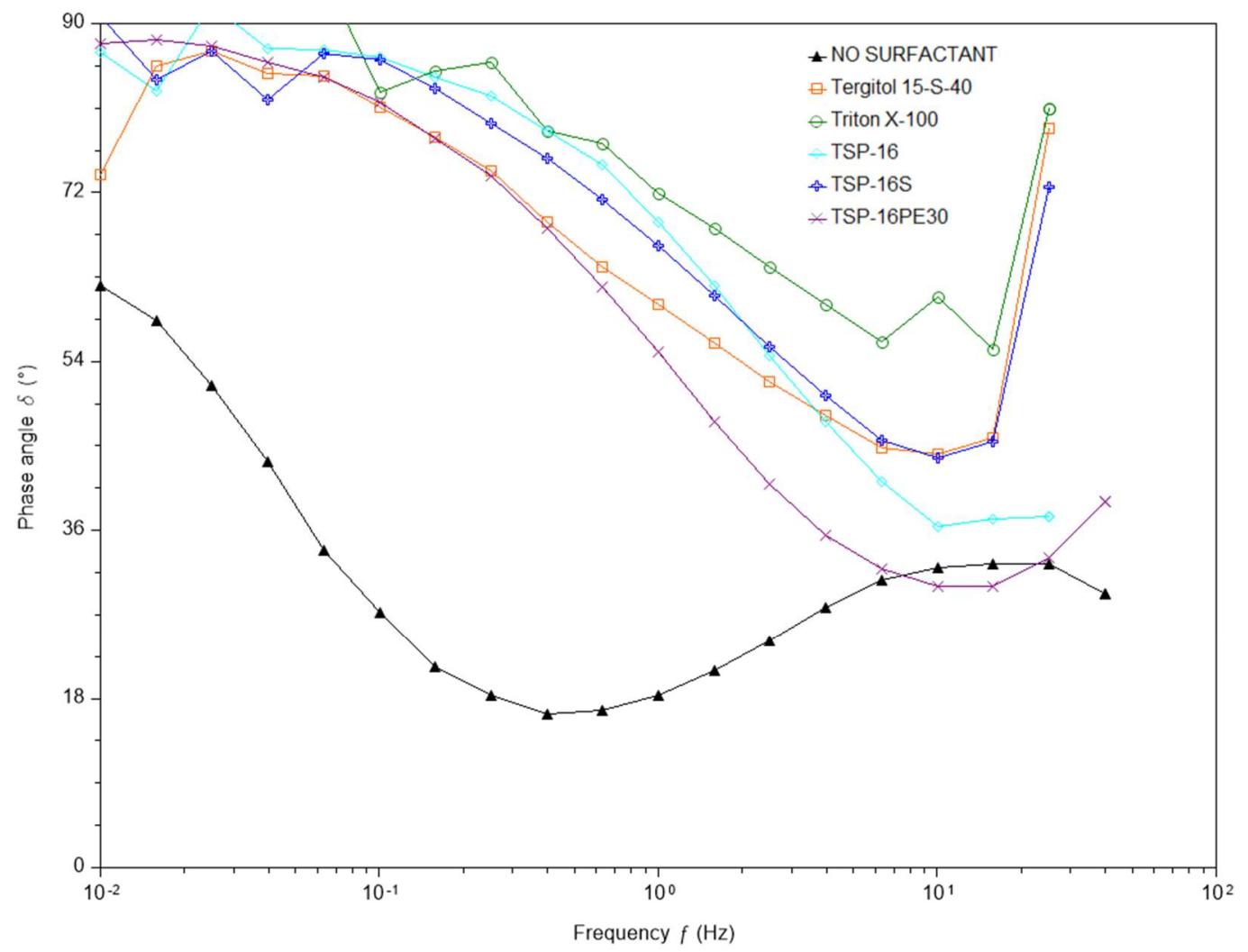

Figure 47. Phase angle plot for BASTY-HEUR-Surfactant system at $1.0 \mathrm{wt} \%$ of additional surfactant.

\subsection{Dynamic Data Analysis of BA/MMA-HEUR-Surfactant systems.}

The BA/MMA systems showed significant differences in the flow sweep analysis. The BA/MMA latex is more hydrophilic than the BA/STY latex and there was a clear difference in the affinity for the HEUR associative ends for the latex surface as was evidenced by the decreased viscosity even at no additional surfactant loading. This decreased affinity of the thickener for associating to the latex particles also meant that surfactant addition led to a disruption of the HEUR associative network at lower surfactant concentrations. This is also shown in the dynamic data analysis in the form of additional strain sweeps and phase angle plots. 
3.5.1 Dynamic Data for BA/MMA-HEUR-Surfactant System with $0.10 \mathrm{wt} \%$ Additional Surfactant

The first difference in the BA/MMA systems can be seen in Figure 48 at $0.1 \mathrm{wt}$. $\%$ surfactant loading. The first distinctive difference is the presence of a storage modulus peak near $50 \mathrm{~Hz}$ in the control sample. With the addition of surfactant however, there is not an immediate rightward shifting of the modulus peak to a higher strain $\%$ than the control. In the case of TSP-16PE30 and partially Triton X-100, there is in fact a shift to a lower strain \%. All systems with additional surfactant do however see an increase in the storage modulus at this concentration. As was previously discussed, it is likely that the addition of surfactant micelles is allowing for a variety of sites for HEUR associative ends to form a network rather than bridges only existing between latex particles. This diverse and extended network should be expected to behave more elastically than a system with only direct bridging like the control. 


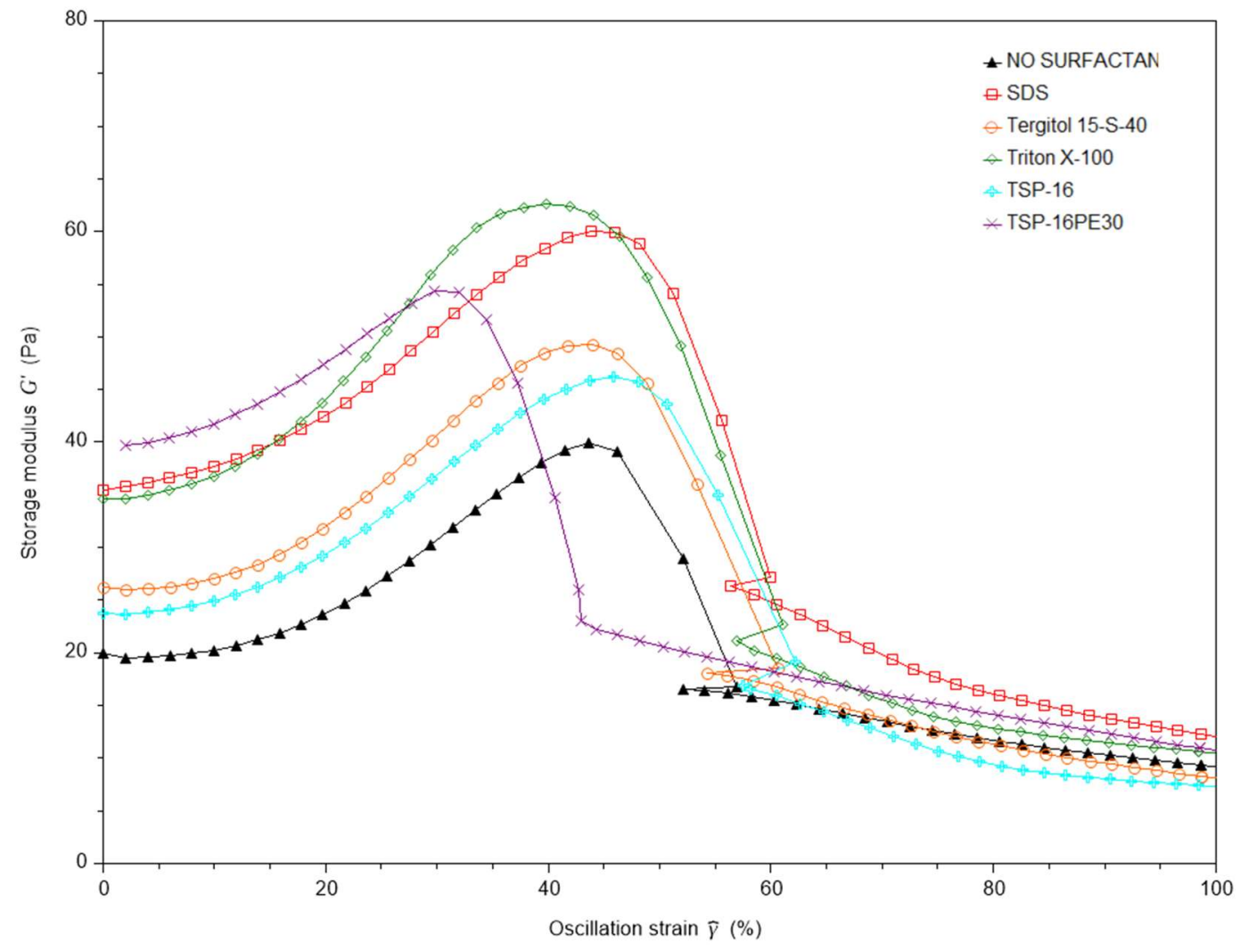

Figure 48. Linear amplitude sweep storage moduli overlay of BA/MMA-HEURSurfactant system at $0.10 \mathrm{wt}$ \% of additional surfactant.

In Figure 49 there is also a noticeable difference in the low surfactant concentration phase angle plot for the BA/MMA systems. First thing of note is the lack of an elastic minimum. Instead of a minimum there is a small plateau-like step starting near $5 \mathrm{~Hz}$ and ending near $12 \mathrm{~Hz}$. Additionally, at $0.1 \mathrm{wt} . \%$ most systems cross over at the plateau of the control. Instead of a return to a more viscous behavior at high frequencies for these systems, they may do not return to a semi-viscous state after this plateau as the BA/STY systems do. Lastly, in the BA/MMA systems SDS and TSP-16PE30 show the largest divergence from the control. The sample with SDS diverging after the crossover point is to be expected, as determined by the high shear viscosity analysis SDS is already at its synergistic viscosifying peak at $0.1 \mathrm{wt} . \%$ loading as was seen in Figure 32 and 
Figure 33. At this concentration in this system, SDS is aiding in the network formation at high shear rates and should be expected to behave similarly at high angular frequencies.

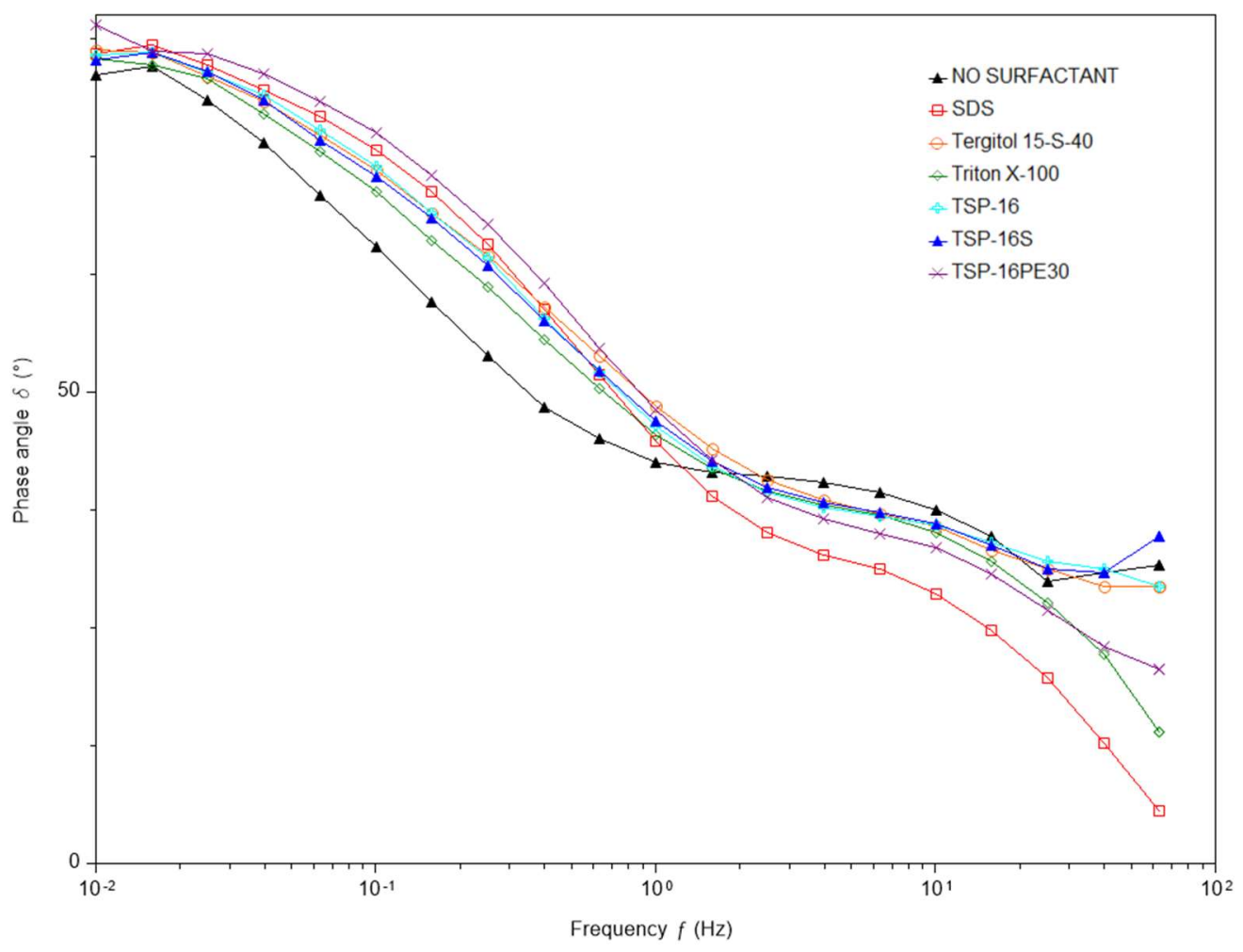

Figure 49. Phase angle plot for BA/MMA-HEUR-Surfactant system at $0.10 \mathrm{wt} . \%$ of additional surfactant.

3.5.2 Dynamic Data for BA/MMA-HEUR-Surfactant System with $0.25 \mathrm{wt} \%$ Additional Surfactant

With an increase to 0.25 wt. \% surfactant loading into the BA/MMA systems we see a return of the familiar behavior seen with higher concentrations of surfactant in the BA/STY latex systems. At this concentration, the sample with SDS already has a decrease in its storage modulus to a near zero value. Also, the other surfactant systems show a rightward shift to higher strain values. However, they do all see a decrease in the 
magnitude of the modulus to values inline with the control. This corresponds to the overall lower intensity of the shear-thickening peaks seen in the flow sweep plots for BA/MMA systems when compared to BA/STY systems. Also corresponding with the flow sweep plots, TSP-16PE30 is now the furthest shifted modulus peak with the highest strain value and lowest storage modulus excluding SDS.

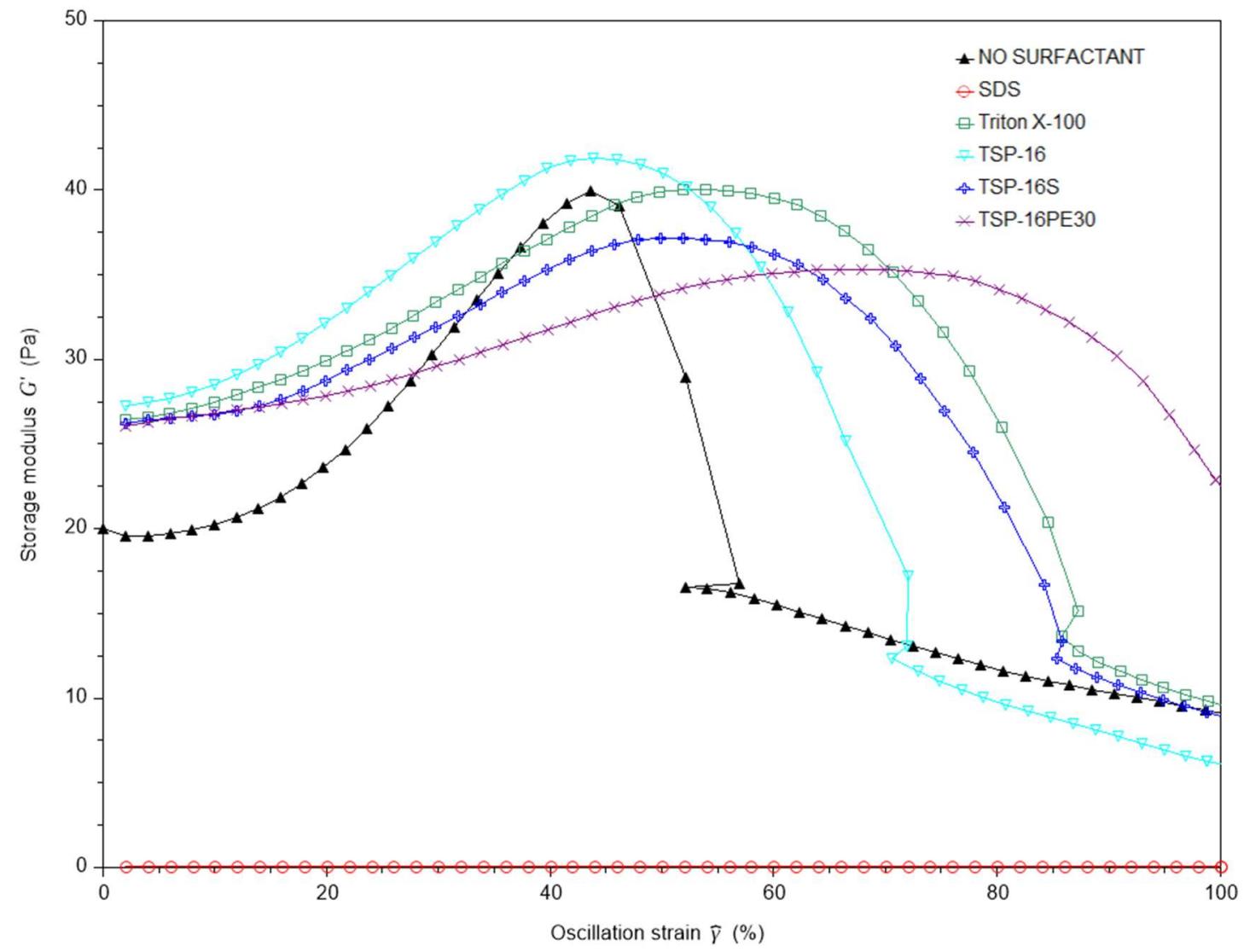

Figure 50. Linear amplitude sweep storage moduli overlay of BA/MMA-HEURSurfactant system at 0.25 wt. \% of additional surfactant.

The phase angle plot for systems with $0.25 \mathrm{wt} . \%$ surfactant loading can be seen in Figure 51. The plot for SDS shows a more viscous behavior than the control at a majority of frequencies. The remaining surfactant systems also show a shift to higher 
frequencies indicating that all systems are behaving more viscously until the crossover point in the step-like region of the control. The systems with SDS and Tergitol 15-S-40 show a transition with an elastic minimum unlike the remaining systems that taper off to a completely elastic response at the highest frequencies. The jagged shape of the SDS curve is due to the low storage modulus values of the sample being at the limit of the instrument's sensitivity.

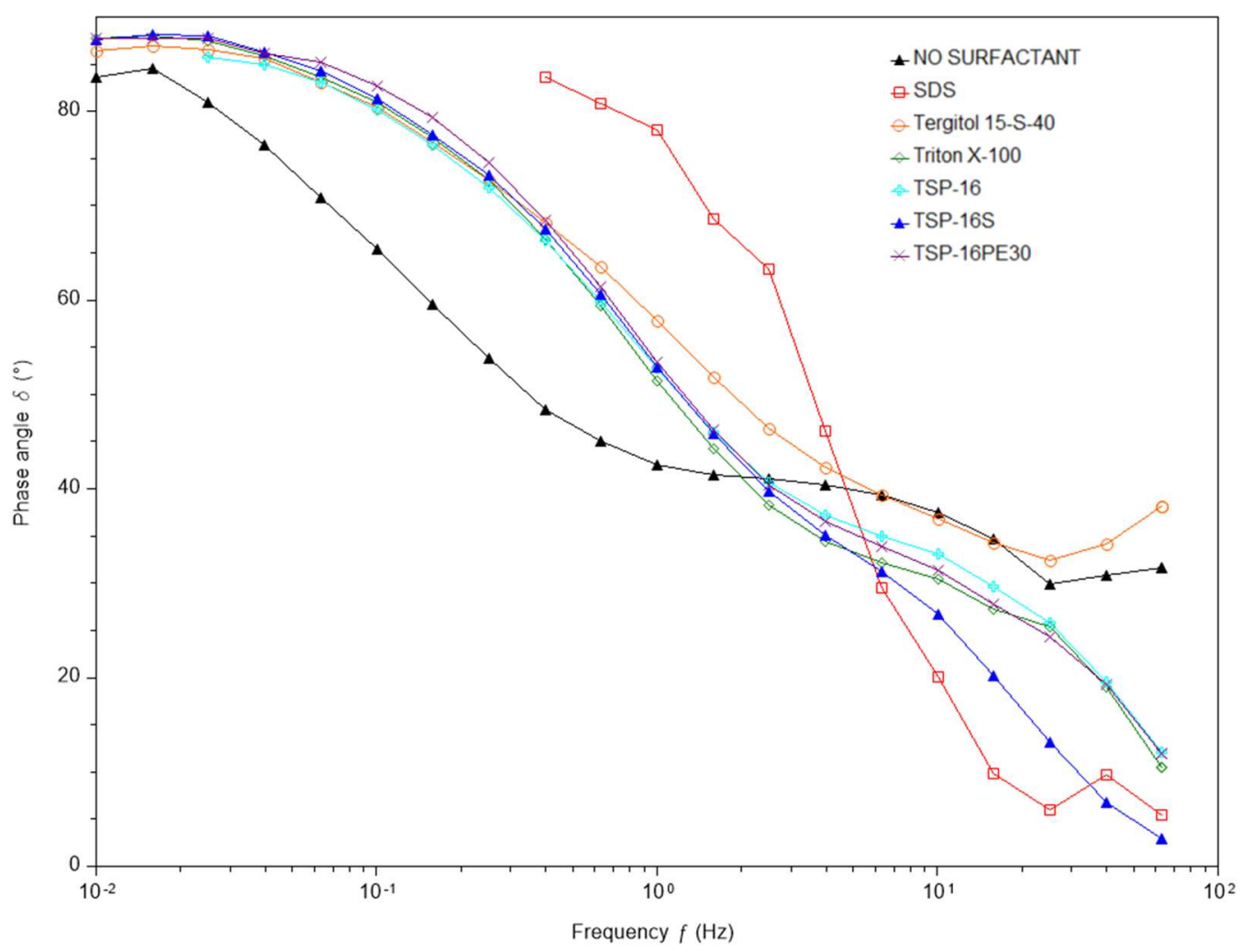

Figure 51. Phase angle plot for BA/MMA-HEUR-Surfactant system at 0.25 wt. $\%$ of additional surfactant. 
3.5.3 Dynamic Data for BA/MMA-HEUR-Surfactant System with $0.50 \mathrm{wt} \%$ Additional Surfactant

With an increase in surfactant concentration to $0.50 \mathrm{wt} . \%$, it is seen that all systems have lost their well-pronounced peak within the $0-100 \%$ strain region as seen in Figure 52. Excluding the system with TSP-16PE30, the remaining systems show a slight increase above $60 \%$ strain indicating that there may be shallow and broad peaks just beyond $100 \%$ strain on the system. These peaks can be resolved in future work. TSP$16 \mathrm{PE} 30$ shows the lowest storage modulus which lines up with the significant drop in viscosity in the flow sweep.

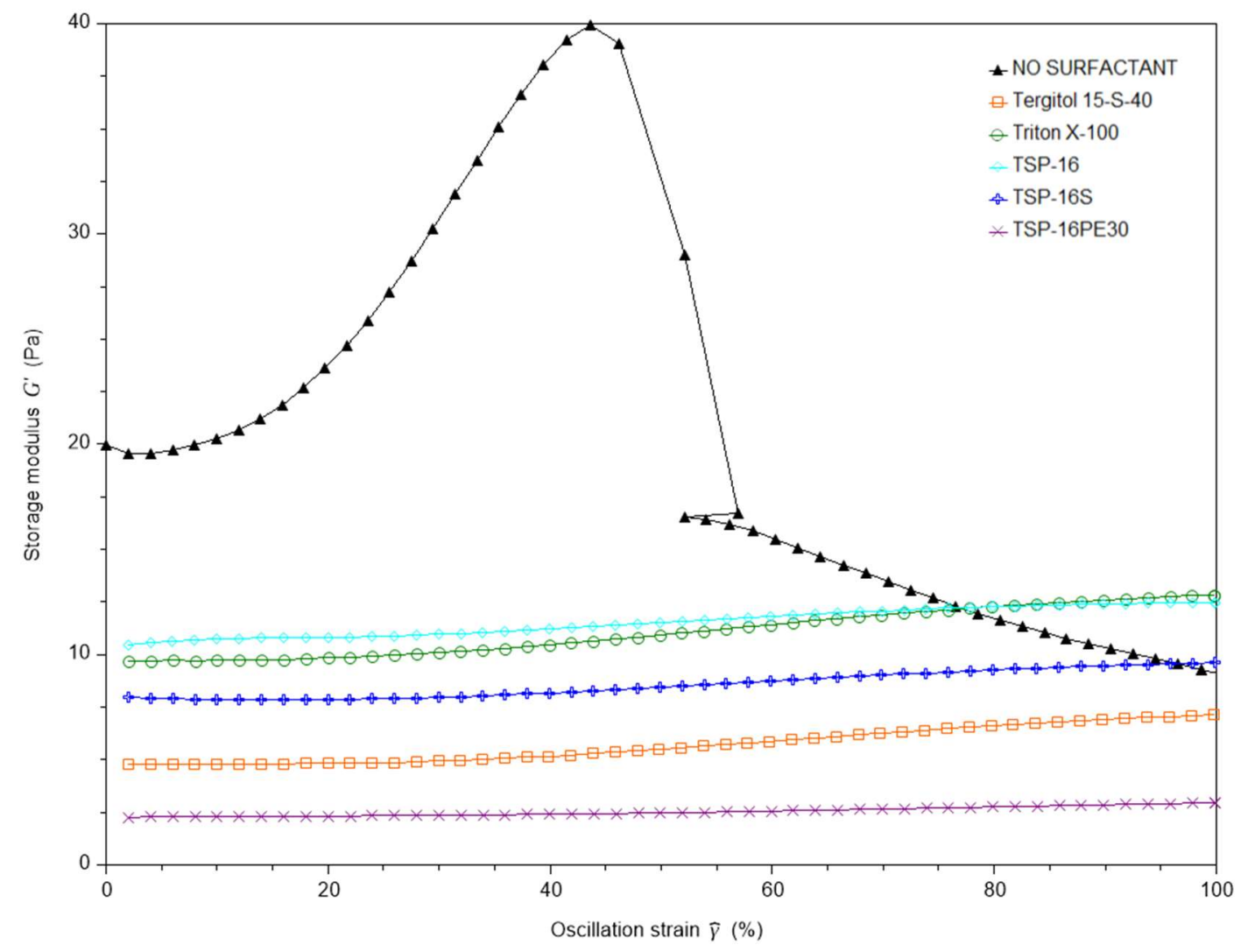

Figure 52. Linear amplitude sweep storage moduli overlay of BA/MMA-HEURSurfactant system at $0.50 \mathrm{wt}$. \% of additional surfactant. 
Figure 53 is the corresponding phase angle plot for the BA/MMA systems at 0.50 wt. \% surfactant loading. The remaining surfactant systems at this concentration are now behaving much like SDS behaved at a lower concentration. These systems remain above a phase angle of $45^{\circ}$ to higher frequencies than the control showing that they are significantly more viscous and fluid-like. Also noteworthy are the changes in behavior seen with Tergitol 15-S-40 and TSP-16. Instead of tapering to a near-zero phase angle, there is now a minimum near $100 \mathrm{~Hz}$ for both of these systems. The remaining surfactant still demonstrate a drop to elastic behavior at this concentration after they cross over the control.

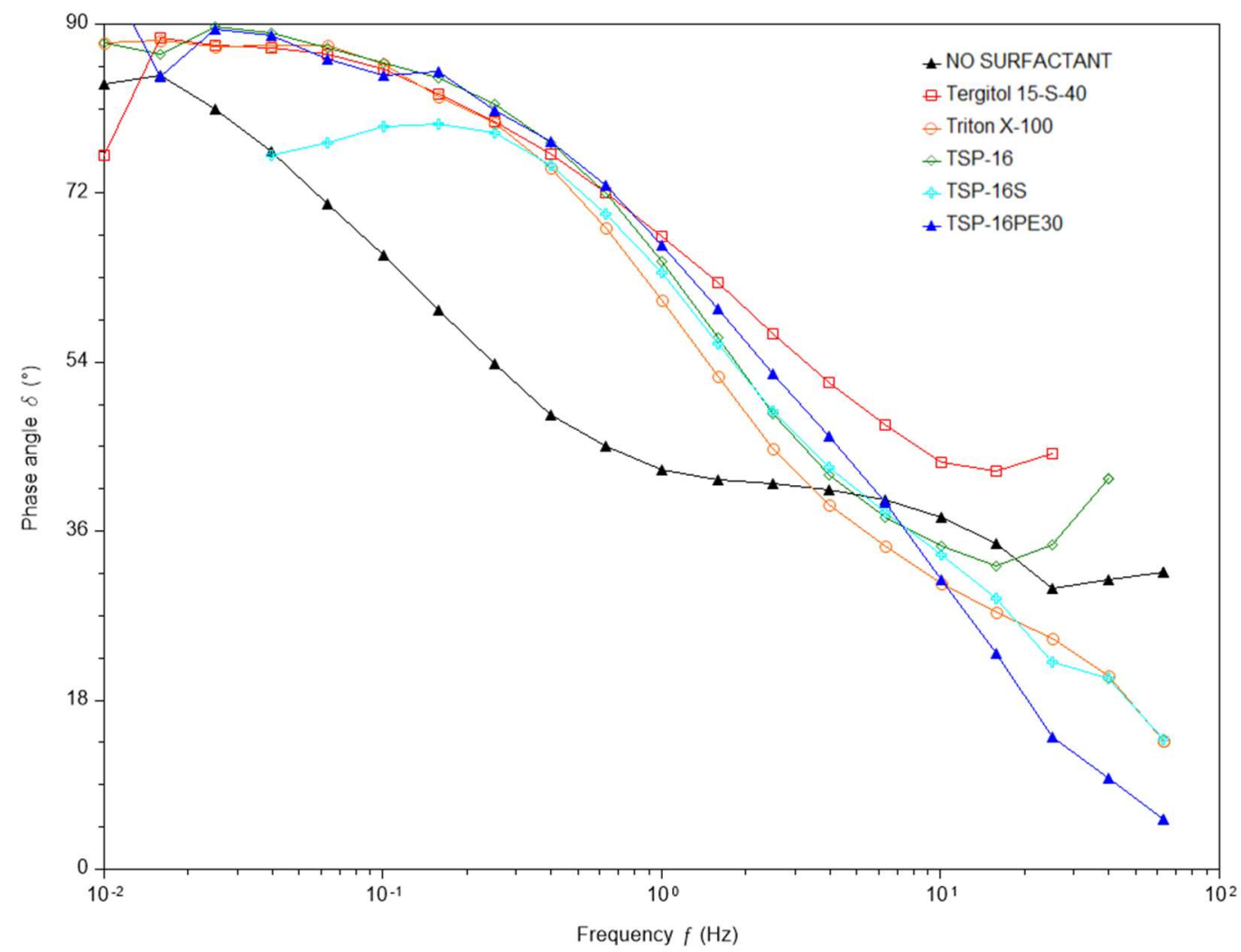

Figure 53. Phase angle plot for BA/MMA-HEUR-Surfactant system at $0.50 \mathrm{wt} . \%$ of additional surfactant. 
3.5.4 Dynamic Data for BA/MMA-HEUR-Surfactant System with $0.75 \mathrm{wt} \%$ Additional Surfactant

The final concentration amplitude sweep was at $0.75 \mathrm{wt} . \%$ concentration of surfactant. This plot shows that all systems have nearly reached a storage modulus value of zero at all frequencies. There are no peaks at this surfactant concentration. Of note however is that the surfactant system with the highest remaining storage modulus is the sample with TSP-16. This also matches with the fact that TSP-16 was the least disruptive surfactant for BA/MMA system flow sweeps. TSP-16PE30 is also the system with the lowest modulus, correlating with the major drop in viscosity seen in the flow sweep analysis.

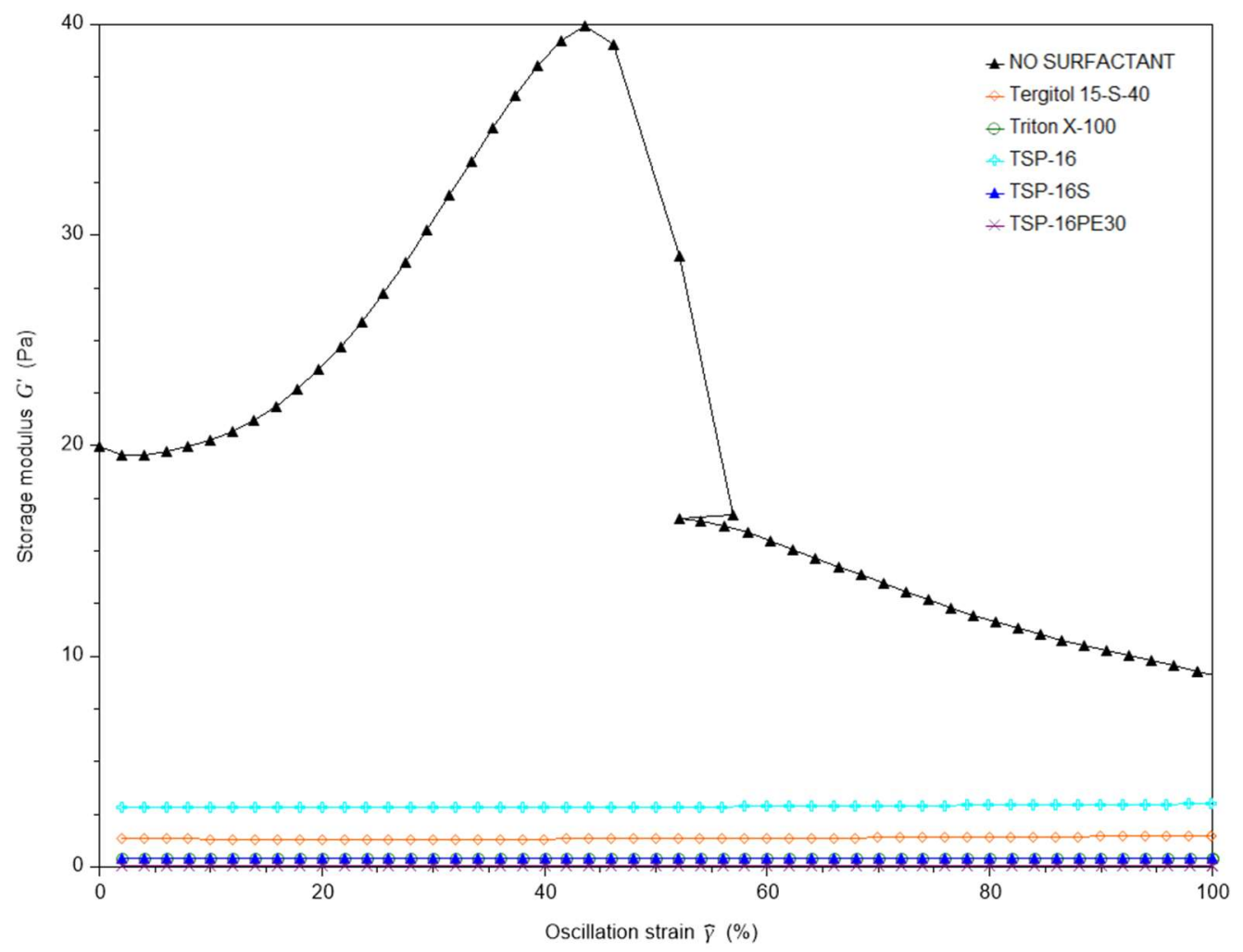

Figure 54. Linear amplitude sweep storage moduli overlay of BA/MMA-HEURSurfactant system at $0.75 \mathrm{wt}$ \% of additional surfactant. 
The final phase angle plot is for these systems with 0.75 wt. \% loading. At this concentration three systems demonstrate a minimum instead of a drop to a phase angle near zero. All systems show a more liquid-like response than the control which is to be expected based on all other data. Triton X-100 demonstrates a unique behavior with a minimum at an approximate phase angle of $70^{\circ}$ instead of lower near $30-40^{\circ}$ like TSP-16 or TSP-16S. However, with these now very viscous systems at high frequencies, it becomes more difficult to acquire data on the rheometer as samples like these can often leave the area beneath the cone.

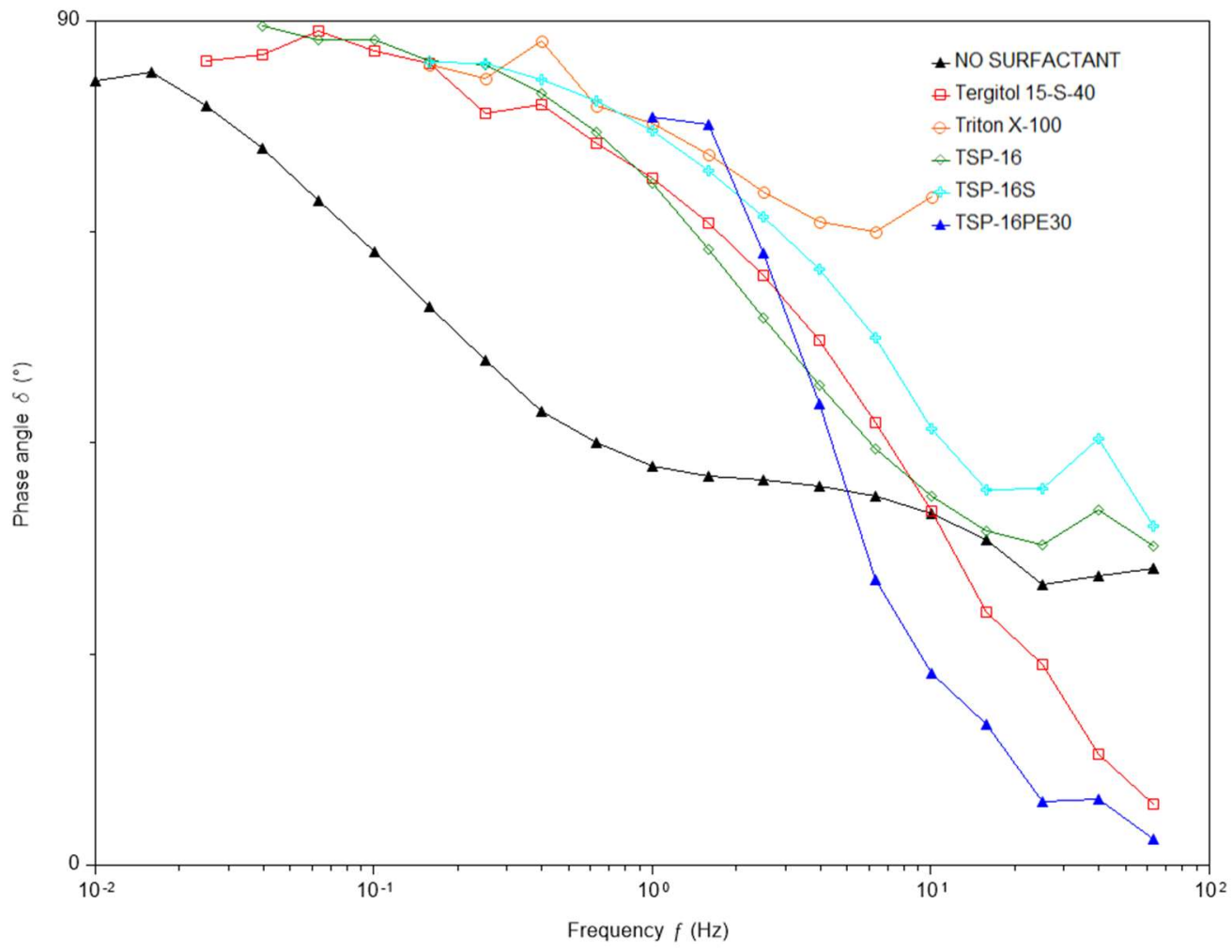

Figure 55. Phase angle plot for BA/MMA-HEUR-Surfactant system at 0.75 wt. $\%$ of additional surfactant. 


\section{CONCLUSIONS AND FUTURE WORK}

Throughout this work, two series of Latex-HEUR-Surfactant systems were explored. The systems in question included a fixed loading of well-characterized HEUR associative thickener. Six surfactants were investigated for these two latex systems at concentrations ranging from 0.0 to $2.0 \mathrm{wt}$ \% loading. In this study there were 3 nonionic surfactants and 3 anionic surfactants. A series of effects were noted from the inclusion of additional surfactant beyond the necessary amount required for latex synthesis and stabilization. These effects were discovered through the use of semi-steady state and dynamic rheological analysis.

The thickener and latex combinations were chosen as systems with particular stability and unique shear-thickening peaks in their flow analysis compared to systems with lower molecular weight HEUR associative thickeners. With the addition of surfactant into these Latex-HEUR systems there were several noted behaviors. Through flow sweep analysis there was a difference noted between the two latex series. Systems with the hydrophilic BA/MMA latex demonstrated a lower overall viscosity than the hydrophobic BA/STY latex systems. The more hydrophilic latex led to a decrease in affinity of the hydrophobic associative ends for the surface of latex particles leading to overall decreased number of viscosifying active bridges.

The second effect discovered through flow analysis was that with the addition of surfactant there was a distinct drop in viscosity. For both latex systems, SDS was noted to have unique behavior which was accounted for based on previously published literature. The remaining surfactants however demonstrated similar behavior to one another for both latex systems. In BA/MMA systems, the surfactant TSP-16PE30 was the most disruptive 
to the associative bridging network. For the hydrophobic BA/STY systems, TSP-16PE30 was the least disruptive surfactant. Triton X-100 was the most disruptive for BA/STY systems and TSP-16 the least disruptive for BA/MMA.

Another phenomenon, aside from the shifting and eventual loss of shearthickening peaks, was the high shear viscosity behavior seen in Regime IV. In this range there was a parabolic relationship seen with increases in surfactant concentrations. Systems with additional SDS were accounted for again with previously published literature. Remaining surfactant systems were found to have a synergistic effect with the HEUR associative networks for viscosifying to a set peak value which was estimated through plots of viscosity at given shear rates. This behavior was also confirmed with previously published literature.

Through more incremental studies of this variable space, these Latex-HEURSurfactant systems could be better understood. There were effects and transitions that were outside of the chosen parameters throughout this body of work. An increase of samples prepared 1.0 and 2.0 wt. \% could reveal several transitions to Newtonian behavior using flow sweep analysis. Keeping in line with work previously published within this research group could also be dramatic increases in surfactant concentrations to levels above 2.0 wt. \% to identify any additional flocculation regions.

Within the scope of this work there was not a hard set effect seen with relation to the $\mathrm{CMC}$ or ionic nature of each surfactant and this is something that can be investigated in future work. Through analysis of several factors such as surface area per molecule, hydrophile-lipophile balance, molecules per surfactant micelle, and micellar volume, a 
better determination of the effects of different surfactant types on Latex-HEUR systems could be made.

Additional work that needs to be explored lies in the dynamic rheological analysis of these Latex-HEUR-Surfactant systems. In at least one BA/STY plot and several plots of the BA/MMA latex there were peaks outside of the chosen experimental range of the work performed. With an extension of experimental parameters on more systems, these peaks could be fully resolved. Further, smaller increments of surfactant concentration could be chosen to fully profile all systems and identify more distinct transitions in fluid behavior, perhaps determining what the critical concentration of surfactant may be for these systems. 


\section{REFERENCES}

(1) Bell, T. J. Development and Implementation of Dispersion Phase Diagrams (DPDs) for Four Different Hydrophobically Modified Ethoxylated Urethane (HEUR) Based Acrylic Paint Systems, Cal Poly State University San Luis Obispo, 2014, Vol. 3. https://doi.org/http://dx.doi.org/110.21043/equilibrium.v3i2.1268.

(2) Wicks, Zeno, Jones, Frank, Pappas, Peter, Wicks, D. Organic Coatings Science and Technology, 3rd ed.; Wiley Interscience: Hoboken, New Jersey.

(3) Herk, A. M. van. Chemistry and Technology of Emulsion, 2nd ed.; Wiley-VCH: Singapore, 2013. https://doi.org/10.1002/978111863852.

(4) Van Dyk, A. K.; Chatterjee, T.; Ginzburg, V. V.; Nakatani, A. I. Shear-Dependent Interactions in Hydrophobically Modified Ethylene Oxide Urethane (HEUR) Based Coatings: Mesoscale Structure and Viscosity. Macromolecules 2015, 48 (6), 1866-1882. https://doi.org/10.1021/ma502174x.

(5) Smith, T. B. Surface Chemistry of Latex Paints: The Effects of Latex and Polymeric Surfactant Interactions on Colloidal Stability and Rheology. California Polytechnic State University, San Luis Obispo, CA, USA 2017.

(6) Milton J. Rosen. Surfactants and Interfacial Phenomena; 2004. https://doi.org/10.1016/0166-6622(89)80030-7.

(7) Winnik, M. A.; Yekta, A. Associative Polymers in Aqueous Solution. Curr. Opin. Colloid Interface Sci. 2010, 2 (4), 424-436. https://doi.org/10.1016/s13590294(97)80088-x. 
(8) Genovese, D. Shear Rheology of Hard-Sphere, Dispersed, and Aggregated Suspensions, and Filler-Matrix Composites. Adv. Colloid Interface Sci. 2012, 171172. https://doi.org/10.1016/j.cis.2011.12.005.

(9) Macosko, C. Rheology: Principles, Measurements, and Applications; Wiley-VCH: New York, 1994.

(10) Han, C. D.; Slattery, J. C.; Geschke, D.; Schramm, G.; Shenoy, a. V.; Saini, D. R.; Publishers, K. A. A Practical Approach to Rheology and Rheometry. Zeitschrift für Phys. Chemie 2007, 199 (Part_1), xxii, 709 p. https://doi.org/10.1017/CBO9781107415324.004.

(11) Smith, T. B. Syneresis and Rheology Mechanisms of a Latex-HEUR Associative Thickener System. 2015, No. December.

(12) Santos, F. A.; Bell, T. J.; Stevenson, A. R.; Christensen, D. J.; Pfau, M. R.; Nghiem, B. Q.; Kasprzak, C. R.; Smith, T. B.; Fernando, R. H. Syneresis and Rheology Mechanisms of a Latex-HEUR Associative Thickener System. J. Coatings Technol. Res. 2017, 14 (1), 57-67. https://doi.org/10.1007/s11998-016-9829-x.

(13) Fernando, R. H.; Dombrowski, G. W.; Rabasco, J. J.; Hartnett, J. P.; Sharpe, B. J. S.; Smith, T. B. Colloidal and Rheological Aspects of Stability of Latex Formulations. In Coatings and Science International Conference; Amsterdam, Netherlands, 2017.

(14) Manion, S. J.; Johnson, L. L.; Fernando, R. H. Shear-Thickening in Aqueous Surfactant-Associative Thickener Mixtures. J. Coatings Technol. Res. 2011, 8 (3), 299-309. https://doi.org/10.1007/s11998-011-9320-7. 
(15) Terri-Chen, T. Using Rheology to Characterize Flow and Viscoelastic Properties of Hydrogels, Adhesives and Biopolymers. Senior Applications Scientist. TA Instruments - Waters LLC 2017, pp 1-58.

(16) Franck, A. Understanding Rheology of Structured Fluids. Book of TA instruments. TA Instruments 2016, pp 1-11.

(17) Butt, H.-J.; Graf, K.; Kappl, M. Physics and Chemistry of Interfaces, Third.; WileyVCH: Darmstadt.

(18) Fellows, C. M.; Doherty, W. O. S. Insights into Bridging Flocculation. Macromol. Symp. 2006, 231, 1-10. https://doi.org/10.1002/masy.200590012.

(19) Braem, A. D.; Prieve, D. C.; Tilton, R. D. Electrostatically Tunable Coadsorption of Sodium Dodecyl Sulfate and Poly(Ethylene Oxide)-b-Poly(Propylene Oxide)-bPoly(Ethylene Oxide) Triblock Copolymer to Silica. Langmuir 2001, 17 (3), 883890. https://doi.org/10.1021/la0013042.

(20) Kontogeorgis, G. M.; Kiil, S. Introduction to Applied Colloid and Surface Chemistry, 1st ed.; John Wiley \& Sons, 2016. https://doi.org/10.1002/9781118881194.

(21) Otsubo, Y. Comparsion of Rheological Behavior of Suspensions Flocculated by Polymer Bridging and Depletion. Nihon Reoroji Gakkaishi 1995, 23 (2), 75-80.

(22) Bower, D. I. An Introduction to Polymer Physics; 2003; Vol. 40. https://doi.org/10.1119/1.1533063.

(23) Auschra, C.; García Romero, I.; Willerich, I.; Reichardt, R.; Muenzenberg, C.; 
Martinez, E.; He, H. The Role of Thickeners in Optimizing Coatings Formulation. BASF Company Ltd: Shanghai, China 2015.

(24) Eley, R. Applied Rheology in the Protective and Decorative Coatings Industry. Br. Soc. Rheol. 2005, 173-240.

(25) Rabasco, J. J.; Saucy, D.; Conley, T. L.; Fasano, D. Rheology Modifier Technology Next-Generation. CoatingsTech 2014, 11 (3), 58-66.

(26) Tulpar, A.; Tilton, R. D.; Walz, J. Y. Synergistic Effects of Polymers and Surfactants on Depletion Forces. Langmuir 2007, 23 (8), 4351-4357. https://doi.org/10.1021/la063191d.

(27) Barmar, M.; Kaffashi, B.; Barikani, M. Investigating the Effect of Hydrophobic Structural Parameters on the Thickening Properties of HEUR Associative Copolymers. Eur. Polym. J. 2005, 41 (3), 619-626. https://doi.org/10.1016/j.eurpolymj.2004.10.007.

(28) Quadrat, O.; Mikešová, J.; Horský, J.; Šňupárek, J. Viscoelastic Behaviour of Butyl Acrylate/Styrene/2-Hydroxyethyl Methacrylate/Acrylic Acid Latices Thickened with Associative Thickeners. Comptes Rendus Chim. 2003, 6 (11-12), 1411-1416. https://doi.org/10.1016/j.crci.2003.09.004.

(29) Kostansek--rohm, E. Using Dispersion / Fiocculation Phase Diagrams to Visualize Interactions Of Associative Polymers, Latexes ,. 2003, 75 (940), 1-8.

(30) Tanaka, F.; Edwards, S. F. Viscoelastic Properties of Physically Cross-Linked Networks: Transient Network Theory. Macromolecules 1992, 25, 1516-1523. 
(31) Beshah, K.; Izmitli, A.; Van Dyk, A. K.; Rabasco, J. J.; Bohling, J.; Fitzwater, S. J. Diffusion-Weighted PFGNMR Study of Molecular Level Interactions of Loops and Direct Bridges of HEURs on Latex Particles. Macromolecules 2013, 46 (6), 2216-2227. https://doi.org/10.1021/ma400114v.

(32) Wang, F.; Peng, J.; Dong, R.; Chang, X.; Ren, B.; Tong, Z. Highly Efficient Hydrophobically Modified Ethoxylated Urethanes (HEURs) End-Functionalized by Two-Tail Dendritic Hydrophobes: Synthesis, Solution Rheological Behavior and Thickening in Latex. Colloids Surfaces A Physicochem. Eng. Asp. 2016, 502, 114-120. https://doi.org/10.1016/j.colsurfa.2016.05.004.

(33) Annable, T.; Buscall, R.; Ettelaie, R. Network Formation and Its Consequences for the Physical Behaviour of Associating Polymers in Solution. Colloids Surfaces A Physicochem. Eng. Asp. 1996, 112, 97-116.

(34) Barmar, M.; Ribitsch, V.; Kaffashi, B.; Barikani, M.; Sarreshtehdari, Z.; Pfragner, J. Influence of Prepolymers Molecular Weight on the Viscoelastic Properties of Aqueous HEUR Solutions. Colloid Polym. Sci. 2004, 282 (5), 454-460. https://doi.org/10.1007/s00396-003-0968-0.

(35) Thickeners, T. A. Association Structure. Langmuir 1993, 9 (4), 1992-1994.

(36) Horigome, M. Long-Time Relaxation of Suspensions Flocculated By. 2002, No. 10, $1968-1973$.

(37) Otsubo, Y. A Nonlinear Elastic Model for Shear Thickening of Suspensions Flocculated by Reversible Bridgin. Langmuir 1999, 15, 1960-1965. 
(38) Kamibayashi, M.; Ogura, H.; Otsubo, Y. Rheology of Complex Suspensions Flocculated by Associating Polymers. J. Rheol. (N. Y. N. Y). 2006, 50 (6), 10091023. https://doi.org/10.1122/1.2358724.

(39) Lukšič, M.; Urbic, T.; Hribar-Lee, B.; Dill, K. A. A Simple Model of Hydrophobic Hydration. J. Phys. Chem. B 2012, 116 (21), 6177-6186. https://doi.org/10.1021/jp300743a.

(40) Mahli, D. M.; Steffenhagen, M. J.; Xing, L.; Glass, J. E. Surfactant Behavior and Its Influence on the Viscosity of Associative Thickeners Solutions, Thickened Latex Dispersions, and Waterborne Latex Coatings. J. Coatings Technol. 2007, 75 (3), 39-51. https://doi.org/10.1007/bf02697720.

(41) Roux, A. H. Thermodynamics in Micellar Solutions : Confirmation of Complex Formation between Sodium Dodecyl Sulfate and Polyethylene Glycol. Langmuir 1997, 13 (21), 1946-1951. https://doi.org/10.1021/la9603961. 